\title{
Mitochondrial activity and dynamics changes regarding metabolism in ageing and obesity
}

\author{
Guillermo López-Lluch
}

Response to reviewers:

First of all, I would like to thanks the reviewers for their excellent revision and the suggested modifications that will reinforce the manuscript.

\section{Reviewer 1.}

Reviewer: This is a timely review on the relationship between mitochondria, metabolism and aging. It includes a very thorough discussion of mitochondrial dysfunction and alterations in mitochondrial dynamics that are thought to occur with aging and/or metabolic stress like high fat diet. It was also relatively clearly written and the figures used were clear, if not a bit overdone.ç

Answer: Thanks for your introduction.

Reviewer: However, this review could improve with some significant changes.

1. The crux of the review as put forth in the title regards "fat metabolism during ageing" which is also reflected in various title headings. However, this reviewer feels this is a bit deceiving based on the review itself as almost none of the review describes mitochondrial regulation of fat metabolism, or how mitochondria in adipose tissue change with age. Rather, all the "metabolic" components are discussions of how mitochrondrial energy function changes with age and or relates to changes in obesity/insulin resistance. A more clear title might be "Mitochondrial activity and dynamics changes regarding metabolism in aging and obesity" with similar changes in subheading titles.

Answer: Thanks for your comments. I have introduced the changes suggested in title and in subheading titles where appropriate.

Reviewer: 2. The author should be cautious with the interpretation of increased fat accumulation with age. To a degree this is true, however, this also plateaus at some point in time and advanced age is generally associated with loss of fat and redistribution of subcutaneous/visceral fat. This also likely contributes to ectopic fat. This is a much more complicated and nuanced definition than just "ageing = increased fat".

Answer: I thanks the comments of the reviewer. This is true, in older old persons a redistribution of fat occurs probably by a last try to maintain the bioenergetic demand at the end of live. I have introduced indications about that 
at the end of the introduction section and try to avoid to indicate that fat accumulates during ageing.

Reviewer: 3. In a similar vein, the author mixes examples of obesity/high fat diet studies with those of aging under the assumption that the same molecular mechanisms are occuring. This is a false assumption and several lines of evidence do show that these are not the same physiological processess, particularly in rodent studies. Organization of the reports by the authors to separate these (and a discussion of how/why they are different) would be important.

Answer: 3.- I separate the evidence that link mitochondrial dysfunction with age or high-fat diet/obesity processes. In the graphical abstract and figure 2 I indicate the major difference in both cases: the accumulation of giant mitochondria during senescence and the disruption of the mitochondrial network in high fat diet/obesity. it is clear that the mechanism is not the same. Ageing is treated in the section of mitochondrial activity and turnover are affected during ageing whereas the changes in fat accumulation are treated in the section about mitochondrial dysfunction is associated with age-dependent fat accumulation. I have removed the indications where both processes were mixed to clarify the message.

Reviewer: 4. Similarly, author makes many comments regarding the relationship between mitochondiral dysfunction and fat accumulation. However, none of the reports presented definitively show cause or effect in this relationship. An author's discussion of whether dysfunction leads to fat or vice versa would be good here.

Answer: Thanks for the comment. In this field, if mitochondrial dysfunction is a cause or a consequence of fat accumulation is not clear. I have introduced some changes and incorporated a section in the conclusion section to indicate this fact and clarify the message.

Reviewer: 5. Some sections (Sestrins particularly) seem a bit disjointed in terms of there is little data regarding their role in metabolic dysfunction, fat accumulation or aging. This is fine, but an entire section on "possible" links seems a little excessive.

Answer: I have introduced the sestrins part in previous section regarding fat accumulation and regulation and removed the complete section.

\section{Reviewer 2}

Reviewer: This is a timely review that discusses the role of mitochondria in aging with focus on fat metabolism. It covers a wide range of mitochondrial biology pertinent to aging. There are a couple of comments to strengthen the manuscript, as follows:

Answer: Thanks for your comments.

Reviewer: [1] It may be worth mentioning mitochondrial signaling, as it is an emerging field with much implications for aging. For instance, the prolongevity 
mitokine FGF21 (reviewed in Pubmed: 26956194) and the age-dependent mitochondrial-encoded peptide MOTS-c and humanin (reviewed in Pubmed: 27518560 and 26956194) in regulating lipid metabolism and diet-induced obesity/insulin sensitivity may be of interest.

Answer: Thanks for your recommendations. The information provided about mitokines and mitochondrial-encoded peptides has been very interesting. I have introduced a section about the importance of these regulators in ageing and fat metabolism.

Reviewer: [2] In addition to 'nutraceuticals', it may strengthen the review by also discussing 'pharmaceuticals' that target pathways mentioned in the manuscript (e.g. mTOR and AMPK) and have strong implications for aging (e.g., rapamycin and metformin).

Answer: Brief indications of these pharmaceuticals have been introduced in the text.

Reviewer: [3] The use of "main" throughout the manuscript is recommended to be replaced with "major" when appropriate. Main implies it is the chief (most important) among other factors, whereas major implies that it is one of several important factors. For instance, in the abstract, "Three main nutritional sensors, mTOR, AMPK and Sirtuins" may unintentionally suggest that these are the three most important ones.

Answer: The change indicated has been introduced in the text. Thanks for your recommendation.

Reviewer: [4] There are numerous grammatical errors that require a thorough revision. Also estrogen and oestrogen are both used in the manuscript.

Answer: Manuscript has been revised in deep and I hope now the grammatical errors have been fixed. 
CALORIC RESTRICTION EXERCISE

NUTRACEUTICALS

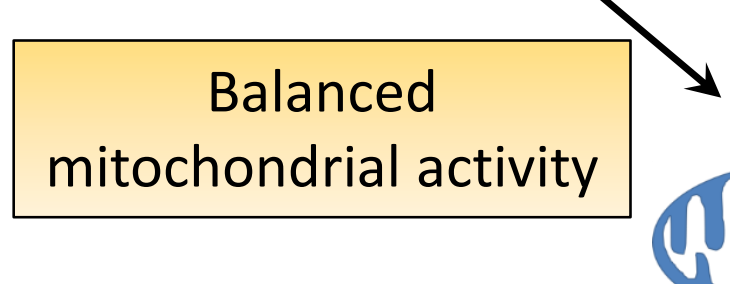

Mitochondrial

Biogenesis

AGING $\longrightarrow$ FISSION

mitochondria
FAT ACCUMULATION

LOW ESTROGENS

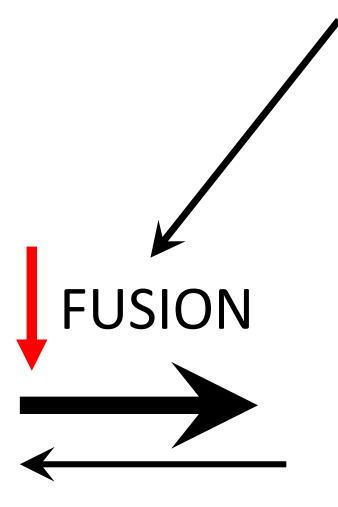

MITO-

— FAT ACCUMULATION

CALORIC RESTRICTION

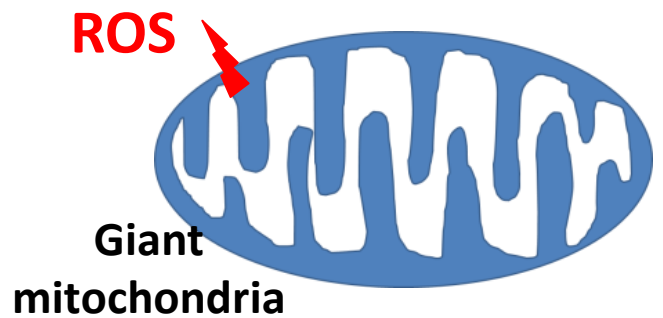

Senescence
Mitochondrial dysfunction $\downarrow$ OXPHOS

$\downarrow \Delta \Psi_{\mathrm{m}}$

$\uparrow$ ROS

EXERCISE

NUTRACEUTICALS
Mitochondrial

turnover 
For: Mech Ageing Dev

\title{
Mitochondrial activity and dynamics changes regarding metabolism in ageing and obesity
}

\author{
Guillermo López-Lluch
}

Universidad Pablo de Olavide, Centro Andaluz de Biología del Desarrollo, CABD-CSIC, CIBERER, Instituto de Salud Carlos III, Carretera de Utrera km. 1, 41013 Sevilla, Spain.

*Address correspondence:

Centro Andaluz de Biología del Desarrollo (CABD), Universidad Pablo de Olavide, Carretera de Utrera Km. 1, 41013 Sevilla, Spain. Email: glopllu@upo.es 


\section{Abstract}

Mitochondria play an essential role in ageing and longevity. During ageing, a general deregulation of metabolism occurs, affecting molecular, cellular and physiological activities in the organism. Dysfunction of mitochondria has been associated with ageing and age-related diseases indicating their importance in the maintenance of cell homeostasis. Three major nutritional sensors, mTOR, AMPK and Sirtuins are involved in the control of mitochondrial physiology. These nutritional sensors control mitochondrial biogenesis, dynamics by regulating fusion and fission processes, and turnover through mito- and autophagy. Apart of the known factors involved in fusion, OPA1 and mitofusins, and fission, DRP1 and FIS1, emerging factors such as prohibitins and sestrins can play important functions in mitochondrial dynamics regulation. Mitochondria are also affected by sexual hormones that suffer drastic changes during ageing. The recent literature demonstrates the complex interaction between nutritional sensors and mitochondrial homeostasis in the physiology of adipose tissue and in the accumulation of fat in other organs such as muscle and liver. In this article, the role of mitochondrial homeostasis in ageing and age-dependent fat accumulation is revised. This review highlights the importance of mitochondria in the accumulation of fat during ageing and related diseases such as obesity, metabolic syndrome or type 2 diabetes mellitus.

Keywords: mitochondria; ageing; fat; gender; inflammation 


\section{Introduction}

Ageing is a natural and progressive process occurring in all living organisms and characterized by the deterioration of structure and functional capacities. Ageing is multifactorial involving different molecular and cellular processes such as genomic instability, telomere attrition, epigenetic alterations, loss of proteostasis, deregulated nutrient sensing, mitochondrial dysfunction, cellular senescence, stem cell exhaustion, and altered intracellular communication (Kennedy et al., 2014; Lopez-Otin et al., 2013). Ageing is also characterized by the appearance of different chronic diseases such as sarcopenia, type 2 diabetes mellitus (T2DM), cardiovascular disease, hypercholesterolemia, hypertension or neurodegenerative diseases among others (Kennedy et al., 2014).

A tendency towards higher visceral adiposity associated with a greater morbidity due to the increase of risk for cardiovascular disease (CVD), hypertension and T2DM has been associated with the progression of ageing (Matsumoto, 2002). Obesity is associated with adipose tissue dysfunction characterized by adipocyte hypertrophy, proinflammatory processes, impaired insulin signalling and insulin resistance (Karakelides et al., 2010). The decrease in the capacity to respond to insulin induces ectopic fat deposition in other organs producing lipotoxicity in muscle, liver and also in pancreas. Thus, the prevalence of obesity in older people has been considered the major cause of metabolic syndrome (Karakelides et al., 2010) and changes in adipose tissue have been recently associated with the development of T2DM (Abranches et al., 2015). Losses of lean mass and shifts to central adiposity have been related 
to functional decline and considered as predictors of mortality (Kirkland et al., 2002).

Fat depots sizes in humans reach a peak by middle and early old age (Cartwright et al., 2007). However, in advanced old age, a decrease in fat tissue occurs although this decrease has been associated with the accumulation of fat in liver, muscle and bone marrow and other organs (Kirkland et al., 2002). In a recent study performed in 70-79 years old men and women, it was determined that loss of weight was accompanied by the reduction of visceral fat mass accompanied by a gain of intermuscular thigh fat area (Santanasto et al., 2016). This redistribution of fat can be related with the preservation of thight muscle since this study demonstrated a strong relationship between loss of thight muscle and mortality whereas no relationship was found with visceral mass (Santanasto et al., 2016).

The role of mitochondria in fat reduction in older old people has been also suggested. In adipose tissue, dysfunctional adipose-like cells appear. These cells are smaller than normal adipocytes and show a low response to insulin. Their dedifferentiation occurs by the decrease of adipogenic factors such as CCAAT/enhancer binding alpha (C/EBPalpha) and peroxisome proliferator activated receptor gamma (PPAR-y), a known mitochondrial regulator (Kirkland et al., 2002).

Mitochondria are key organelles in the metabolism of eukaryotic cells (LopezLluch et al., 2008). Their activity is modulated by the availability of energy in cells. Key regulators of metabolism not only affect the activity of mitochondria but also their dynamics and turnover. One of these regulators is the mammalian Target of Rapamycin (mTOR) which is activated by high calorie intake or high 
levels of aminoacids (Laplante and Sabatini, 2009). Opposite regulators are AMP-activated kinase (AMPK) and sirtuins that are activated when energy uptake is limited (Hardie, 2011).

The term mitochondrial dysfunction is widely used in bioenergetics although there is not a precise definition. Any abnormality in the key physiological functions of mitochondria: generation of ATP, detoxification of ROS and ROSrelated damage, regulation of cytosolic calcium levels, synthesis of lipids, etc., can be considered as mitochondrial dysfunction (Brand and Nicholls, 2011). Mitochondrial dysfunction is associated with the functional decline found in tissues and organs during ageing (Riera et al., 2016) and, for this reason, it has been associated with a number of age-related diseases such as neurological disorders, CVD, liver and kidney diseases, diabetes and cancer (Lane et al., 2015; Payne and Chinnery, 2015; Ryan et al., 2015; Wallace, 2012). Recently, a study carried out with 248 non-diabetic participants from the Baltimore Londigutinal Study of Aging has associated impaired mitochondrial capacity with greater insulin resistance (Fabbri et al., 2016).

Information about the role of mitochondria on white adipose tissue (WAT) is limited in animal models and humans. Since the regulation of mitochondrial activity in WAT can be associated with the accumulation of fat and a putative disruption of mitochondrial physiology, the study of mitochondrial homeostasis is important to develop strategies or therapies to avoid or delay the progression of fat accumulation-derived diseases. For this reason, in this review, the role of mitochondrial activity, dynamics and turnover on fat accumulation in WAT, muscle or liver and the molecular factors involved in these processes is reviewed in deep. 


\section{Molecular mechanisms involved in the regulation of mitochondrial physiology.}

Mitochondrial physiology, dynamics and turnover respond to the relationship between the activities of key regulators of cell metabolism. In high calorie intake situations, mTOR, an ubiquitous serine/threonine kinase involved in cell growth and the control of metabolism, is activated. This regulator is a complex of different proteins and regulates different pathways involved in angiogenesis, tumour development, insulin resistance, adipogenesis and activation of immune cells (Laplante and Sabatini, 2009). It is present in two different complexes, mTOR complex I (mTORC1) and mTOR complex II (mTORC2). Both complexes promote the transcription of genes involved in carbohydrate metabolism and lipogenesis, enhance protein translation by activating ribosomal protein 6 kinase (S6K) and, at the same time, downregulate catabolic processes through inhibiting the transcriptional activity of the forkhead box transcription factors family (FoxO) and autophagy (Duvel et al., 2010; Wullschleger et al., 2006).

The signalling pathway triggered by mTOR competes against other regulators of the cell metabolism such as AMPK and sirtuins, NAD+-dependent deacetylases that induce more efficient energy consumption in situations of low intake, starvation or calorie restriction (CR), (Fig. 1). AMPK is one of the major sensitive energy sensors in cells and organisms. This kinase is activated in response to the increase of the AMP/ATP ratio, for example, after deprivation of 
glucose, whereas its activity is reduced when cells are full of energy and the AMP/ATP ratio decreases (Hardie, 2011).

Another of the most important sensors of energy in cells and regulators of mitochondrial activity are sirtuins. This family of $\mathrm{NAD}^{+}$-dependent deacetylases is found in all the organisms, from prokaryotes to humans, and regulates the activity of many enzymes by removing acetyl residues, except in the case of mammalian SIRT4 and SIRT5 that show ADP-ribosyl-transferase activity (Santa-Cruz Calvo et al., 2012). Sirtuins also act as metabolic sensors by detecting fluctuations in the $\mathrm{NAD}^{+} / \mathrm{NADH}$ ratio in cells. When the amount of nutrients decrease, especially in the case of glucose, levels of NAD+ increase and sirtuins are then activated. The number of proteins regulated by deacetylation has increased enormously from their discovery. The importance of these proteins in the regulation of metabolism is increasing since they are involved in different key aspects of cell physiology from cell cycle control to metabolism and antioxidant protection (Santa-Cruz Calvo et al., 2012). However, the most studied aspect of Sirtuins has been their role in longevity (Guarente, 2011, 2013). In mammals, CR induces the expression and the activity of sirtuins in many organs and their activities have been associated with most of the metabolic effects produced by CR (Imai and Guarente, 2010). For example, SIRT1 has been suggested to play an important role since this enzyme activates eNOS by deacetylation in human vascular cells (Mattagajasingh et al., 2007). Further, it has been demonstrated that eNOS is induced by CR in mice and human muscle (Civitarese et al., 2007; Nisoli et al., 2005) being essential in the CR-induced mitochondrial biogenesis since in eNOS--- mice this induction is blocked (Nisoli et al., 2005). 
The peroxisome proliferator-activated receptor gamma coactivator 1a (PGC1a) is the central factor involved in the regulation of mitochondrial mass and the adaptation to energy demand since this transcriptional coactivator stimulates mitochondrial biogenesis, regulates mitochondrial dynamics, modulates oxidative phosphorylation and controls mitochondrial genome copy number (Gouspillou et al., 2014; Handschin and Spiegelman, 2006). Importantly, AMPK and SIRT1, two major metabolic sensors in cells, modulate directly PGC1a activity through phosphorylation and deacetylation respectively (Jager et al., 2007; Nemoto et al., 2005). Thus, SIRT1 and AMPK modulate PGC1a activity at the transcriptional and post-translational level (Martin-Montalvo and de Cabo, 2013) (Fig. 1).

Into the sirtuins family, SIRT3, a member located in the mitochondria, seems to a very important role in the regulation of mitochondrial activity especially in fat metabolism (Kendrick et al., 2011). SIRT3 levels increase in skeletal muscle after $\mathrm{CR}$ and decrease under high-fat diet feeding indicating a regulation depending on the calorie intake (Palacios et al., 2009). SIRT3 has been also involved in the regulation by deacetylation of electron transport chain complex II activity (Kendrick et al., 2011), modulation of the activity of Mn-SOD (Qiu et al., 2010) or regulation of autophagy by deacetylation of FOXO3 (Kume et al., 2010). Furthermore, SIRT3 KO mice show repression of PGC1a down regulation and low levels of AMPK phosphorylation after CR indicating a key role of this mitochondrial sirtuin in metabolic adaptation to calorie intake (Palacios et al., 2009). 
Interestingly, these mechanisms regulate each other in all the organisms studied in an evolutionary conserved response that avoids fruitless energy expenditure and use recycled structures to maintain organisms' survival.

\section{Regulation of AMPK and mTOR controls energy metabolism.}

Control of mitochondrial biogenesis and turnover through AMPK and MTOR activities is key in the regulation cell metabolism and, in particular, mitochondria. AMPK stimulates cellular catabolism of sugars, proteins and lipids. AMPK inhibits by phosphorylation several anabolic enzymes such as acetyl-coenzyme A (CoA) carboxylase (ACC), glycerol phosphate acyl transferase (GAPT), 3-hydroxyl-3-methylglutaryl CoA reductase (HMGCR) and glycogen synthase (GS) affecting glycogen and lipid biosynthesis (Hardie et al., 2012). At the same time, AMPK also inhibits the transcriptional activity of sterol regulatory element binding protein (SREBP) decreasing the expression of genes involved in fatty acid synthesis (Li et al., 2011). But, more importantly, AMPK inhibits mTORC1 activity both directly (Gwinn et al., 2008) or indirectly (Inoki et al., 2003).

One of the most important aspects of AMPK and mTORC1 regulation is their contrary effect on autophagy and mitophagy. Whereas mTORC1 negatively regulates autophagy through phosphorylating the mediators, unc-51-like kinase 1/2 (ULK-1 and ULK-2), in inhibitory sites (Chan, 2009; Kim et al., 2011), AMPK positively regulates autophagy by phosphorylating these proteins in their activating sites (Alers et al., 2012). The regulation of Forkhead box proteins (FoxO) plays an essential role in mito- and autophagy. These proteins are a 
family of transcription factors playing key roles in the regulation of cell growth, proliferation, differentiation and longevity. FoxO are involved in the induction of several stress response genes (Goto and Takano, 2009) highlighting their importance in the induction of protective mechanisms in the organisms. In fact, the regulation of FoxO proteins during ageing and their modulation by metabolic regulators such as mTOR, AMPK and sirtuins have associated these proteins with the protective effect of $\mathrm{CR}$, polyphenols or exercise against mitochondrial damage by regulating autophagy and antioxidant protection preventing by these

mechanisms the progression of ageing-related disease (Lopez-Lluch and Navas, 2016) (Fig. 1).

\section{Mitochondrial activity and turnover are affected during ageing.}

Mitochondria play a key role in the pathophysiology of ageing and in earlier stages of the events leading to the ageing phenotype (Gonzalez-Freire et al., 2015). Some evidence indicate that ageing is associated with the accumulation of deficient mitochondria that produce a significant increase of ROS generation (Chistiakov et al., 2014; Merry, 2002). In human muscle, ageing is accompanied by a higher need of mitochondria to maintain the same respiratory capacity as in young people indicating decay in mitochondrial efficiency (Larsen et al., 2012). Although the role of mitochondria as the major source of intracellular ROS production in ageing has been questioned during the last years (Gram et al., 2015; Payne and Chinnery, 2015), most data suggest that there is an increase in oxidative damage in the elderly affecting senescence (Lu and Finkel, 2008), fertility declining (Benkhalifa et al., 2014), skin ageing (Kammeyer 
and Luiten, 2015), neutrophils' activity (Kovalenko et al., 2014) or skeletal muscle (McDonagh, 2016).

Due to the dual role of ROS as damaging and signalling factors, a moderate level of ROS production during life can be beneficial by inducing hormetic responses that reinforce the capacity of the organism to respond to stronger injury (Rattan, 2008). In model organisms, a recent study in Drosophila indicates that ROS increase with age as mitochondrial function deteriorates but high levels of ROS during the life of the organism seems to act as secondary messengers inducing endogenous protective mechanisms in the animals that can increase lifespan (Scialo et al., 2016). If ROS production is balanced with antioxidant enzymes, oxidative damage did not occur, but if these enzymes decay during aging, as has been found in different models (Rodriguez-Bies et al., 2015; Rodriguez-Bies et al., 2016; Tung et al., 2014; Tung et al., 2015b), oxidative damage accumulates.

In any case, evidence indicate that damaged mitochondria show low efficiency producing lower ATP and higher release of ROS through a deficient activity of the mitochondrial electron transport chain complexes. Near all the complexes of the mitochondrial electron transport chain are involved in ROS production and it has been recently shown that, the assembly of these complexes in supercomplexes seems to regulate ROS production (Goncalves et al., 2015). Thus, a progressive deterioration of supercomplexes during ageing could be the cause of an irreversible increase in ROS in older organisms (Genova and Lenaz, 2015). 
Accumulation of damaged mitochondria during ageing is also associated with the decline of the mitochondrial turnover through inhibiting mitophagy; a specific autophagy process that removes damaged mitochondria (Chistiakov et al., 2014). The correct activity of the quality control of mitochondria is considered a crucial factor in counteracting ageing process (Weber and Reichert, 2010). Renovation of the mitochondrial network plays a key role in health-span increase after CR (Lopez-Lluch et al., 2008) indicating that the control of the accumulation of damaged mitochondria is an important factor in the progression of ageing. In fact, prolongevity factors such as $\mathrm{CR}$, exercise and polyphenols prevent the accumulation of damaged mitochondria at least in part by inducing mito- and autophagy (Lopez-Lluch and Navas, 2016) (Fig. 2).

Another factor involved in the decay in mitochondrial turnover during ageing is the impairment of mitochondrial biogenesis, especially in high energy demanding tissues such as muscle, brain or heart (Conley et al., 2000; Short et al., 2005). As in the case of induction of autophagy, ageing delaying interventions such as $\mathrm{CR}$, exercise or nutraceuticals as resveratrol also induce mitochondrial biogenesis in humans and other organisms (Baur et al., 2010; Feige et al., 2008; Rodriguez-Bies et al., 2015). Thus, it seems clear that maintenance of mitochondrial turnover removing ROS-producing damaged mitochondria at the same time that biogenesis produces new components is essential in the prolongevity effects of these factors.

The bioenergetic state of the cell is directly related with the regulation of mitochondrial dynamics; the balance in fusion/fission processes occurring in the mitochondrial network. Mitochondrial fusion is associated with optimized mitochondria whereas fission is associated with damaged mitochondria and 
their removal by mito- or autophagy (Lopez-Lluch et al., 2008). Mitochondrial dynamics are regulated by a group of proteins that regulate fusion and fission in mitochondria. The major proteins involved in fusion are mitofusins 1 and 2 (Mfn1 and Mfn2) and autosomal dominant optic atrophy-1 (OPA1) (Wai and Langer, 2016). All these proteins are large GTPases localized in mitochondria. Mitofusins are located at the outer membrane of mitochondria acting in the fusion of these membranes whereas OPA1 is located in the intermembrane space and acts in the fusion of the inner membranes. Mfn2 is also associated in the connection between mitochondria and the endoplasmic reticulum (ER) playing a role in the development of endoplasmic reticulum (ER) stress and fat accumulation in conditions of metabolic stress (de Brito and Scorrano, 2008, 2009).

Fission segregates components of the network that are irreversibly damaged or unnecessary for subsequent removal (Twig et al., 2008). The major proteins involved in mitochondrial fission are dynamin related protein 1 (DRP1) and fission protein 1 (FIS1). DRP1 is also a GTPase protein located mainly in the cytosol although a fraction is localized on mitochondria (Smirnova et al., 2001). DRP1 is recruited on the outer mitochondrial membrane by FIS1 which is already inserted in this membrane (Santel and Frank, 2008; Yoon et al., 2003). However, other factors such as mitochondrial fission factor (Mff) or mitochondrial dynamics proteins of 49 and $51 \mathrm{kDa}$ (MiD49 and MiD51) can also mediate DRP1 recruitment in absence of FIS1 (Loson et al., 2013; Shen et al., 2014), although mutations in FIS1 disrupt downstream mitochondrial degradation events indicating the importance of this protein in mitochondrial turnover (Shen et al., 2014). 
During ageing, the equilibrium of fusion and fission is disturbed producing the accumulation of damaged mitochondria. Blocking the fission process by depleting FIS1 or blocking DRP1 activity leads to cell senescence in human cells accompanied by the accumulation of damaged mitochondria showing lower mitochondrial potential and higher ROS production (Lee et al., 2007; Park et al., 2010). In fact, senescence has been associated with the accumulation of giant dysfunctional mitochondria characterized by highly interconnected networks and ultrastructural abnormalities in human lymphocytes and mice cells (Beregi and Regius, 1987) accompanied by higher production of ROS and lower mitochondrial respiratory activity (Yoon et al., 2006). These facts highlight the importance of a functional fission machinery to discard damaged mitochondria by autophagy or mitophagy (Gomes and Scorrano, 2008; Twig et al., 2008).

It is not clear, how different morphologies affect mitochondrial function, although changes in mitochondrial shape during development suggest a close relationship between morphology and function. Placenta, skeletal muscle, vascular smooth cells and neurons suffer mitochondrial defects by deficiencies in mitochondrial dynamics (Chen and Chan, 2005). Further, RNAi studies have demonstrated an inverse relationship between fusion capacity and respiration rates mainly affecting complexes I, III and IV in mammals and humans (Chen et al., 2005). This effect was reversed by restoring Mfn and OPA1 proteins and was not a consequence of changes in biogenesis regulators such as PGC1a, indicating a direct relationship of mitochondrial dynamics and regulation of OXPHOS subunits activities (Chen et al., 2005). In other organs, defects in mitochondrial fusion/fission dynamics are also essential for the quality control of 
mitochondria in many age-related diseases as in the case of cardiac homeostasis and senescence (Biala et al., 2015), or in neurodegeneration and hypothalamic dysfunction (Zorzano and Claret, 2015). On the other hand, it has been recently proposed that a decrease in the levels of mitochondrial fission proteins can be an adaptive response to increase the resistance against oxidative stress promoting a resistance phenotype in response to a higher ROS production by mitochondrial dysfunction (Mai et al., 2010) or even a defensive response to maintain functional cell mitochondria (Chen and Chan, 2005). Thus, the decrease of fission process would allow mitochondria to escape autophagic degradation maintaining enough ATP production even with deficient mitochondria (Gomes and Scorrano, 2011). All these evidence found in mammals models and humans indicate that dysfunction in the control of mitochondrial dynamics can be associated with the accumulation of damaged mitochondria during ageing in different organs including adipose tissue.

Accumulation of damaged mitochondria during ageing increases the production of ROS that can not only produce damage in molecules and structures but also modulate cell responses such as antioxidant response, removal of damaged molecules, induction of organelle turnover and regulation of mitochondrial activity (Ristow, 2014). This means that the balance between ROS production and the capacity of the cells to respond to ROS levels could affect cell homeostasis during ageing. Low levels of ROS have been also involved in the modulation of glucose metabolism and the increase in oxidative metabolism and stress resistance in CR conditions or after exercise indicating a role of ROS in the regulation of metabolism (Barbieri et al., 2013). Moreover, in a model of longevity in Drosophila it has been recently demonstrated that moderated levels 
of ROS can induce prolongevity effects (Scialo et al., 2016) by activating recycling mechanisms such as mitophagy and autophagy (Weber and Reichert, 2010). Although the ROS-dependent mechanisms involved in the increase of mitochondrial metabolism have to be clarified, it seems that the AMPKdependent modulation of FoxO-dependent autophagy and PGC1 $1 \alpha$-regulated mitochondrial biogenesis are involved ( $\mathrm{Li}$ et al., 2015). In resume, a moderate production of ROS accompanied by the maintenance of the capacity to respond to them is enough to maintain the population of mitochondria in a balanced status, creating new mitochondria and eliminating damaged components.

\section{Mitochondrial turnover can be regulated by different factors during} ageing.

Control of mitochondrial dynamics including turnover seems to play an essential role in ageing. Multiple factors affect mitochondrial activity and many of them have been associated with cell senescence and ageing as has been recently reviewed (Ziegler et al., 2015). Unfortunately the factors that control the change in mitochondrial dynamics during ageing have not been determined yet (Collins et al., 2002). The identification of these factors will be essential to understand if they are dysregulated during ageing or in pathological situations. Among them PGC1 $\beta$ can be one of the candidates since this transcription factor control basal mitochondrial biogenesis and stimulates mitochondrial fusion through the modulation of Mfn2 (Liesa et al., 2008) (Fig. 2).

Another interesting candidate is the mitochondrial E3-ubiquitin ligase 1 (Mul1), a mitochondrial membrane protein showing different functions (Peng et al., 
2016). Mul1 acts as an ubiquitin-ligase that ubiquitinate proteins such as Mfn2, p53 or ULK1, leading to their degradation. Further, Mul1 also acts as small ubiquitin-like modifier (SUMO) E3-ligase, sumoylating other proteins such as DRP1 enhancing its stabilization. Then, Mul1 controls mitochondrial dynamics by inducing fission at the same time that reduce fusion by inducing Mfn2 degradation (Peng et al., 2016). In fact, Mul1 has been associated with the fragmentation, depolarization and clearance of mitochondria through mitophagy in a mechanism regulated by FoxO (Lokireddy et al., 2012). Furthermore, the activity of this ubiquitin ligase has been recently associated with the maintenance of muscle mass (Palus et al., 2014).

Further, SIRT3 also emerges as an important factor in the control of mitochondrial dynamics during ageing since it deacetylates OPA1 elevating its GTPase activity and then, increasing fusion processes (Samant et al., 2014). For further information, this mechanism and others have been recently reviewed in deep in its role in ageing and ageing-related diseases (Benigni et al., 2016).

Recent research has found another component in the control of cell metabolism and mitochondrial activity, sestrins. Sestrins are another highly conserved protein family encoded by genes known as Sens that are upregulated after environmental stress, hypoxia and DNA damage (Budanov and Karin, 2008). Whereas invertebrates' genome contains only a single sens gene, vertebrates express three sestrins (Sens1-3). Their importance in metabolism regulation resides in their regulation of mTORC1 activity. Sestrins are able to bind AMPK activating it and, by this mechanism, repress mTORC1 activity (Budanov and Karin, 2008). The prolongevity effect of sestrins have been recently proposed after the experiments performed in dSens-deficient $D$. melanogaster (Lee et al., 
2010) and in C. elegans (Yang et al., 2013). In mice, these proteins have also been associated with the regulation of metabolism producing a prolongevity effect (Lee et al., 2012).

If sestrins expression decays during ageing, accumulation of damaged mitochondria will increase due to reduction of mito- or autophagy. In agreement with this hypothesis, it has been recently demonstrated that the protective effect of sestrins depends on the promotion of autophagic degradation of Keaps 1 and Nrf2 activation by inhibition of mTORC1 (Rhee and Bae, 2015) probably by eliminating damaged mitochondria as in the case of rat kidney cells (Ishihara et al., 2013). The same mechanism has been associated with the protective effect of sestrin2 in rodent dopaminergic neurons against rotenone toxicity (Hou et al., 2015). Future research about these proteins and its role in the control of metabolism in muscle, adipose tissue and liver during ageing will clarify their importance in the accumulation of fat, especially in humans (Fig.2).

Interestingly, other factors, such as mitochondrial signaling peptides have been recently described to mitochondrial homeostasis. Defects in mitochondrial activity activate a retrograde response that communicat mitochondrial dysfunction to nucleus through activation of AMPK/PGC1a signalling axis (Quiros et al., 2016). The short open reading frame found within the mitochondrial 12S rRNA known as MOTS-C (mitochondrial open reading frame of the $12 \mathrm{~S}$ rRNA-c) appears mainly in skeletal muscle and activate AMPK (Lee et al., 2016). Interestingly, treatment with MOTS-C in mice prevent agedependent and high-fat diet induced insulin resistance and obesity indicating an important role of mitochondria in the control of fat metabolism probably producing prolongevity effects (Fuku et al., 2015; Lee et al., 2015). Other 
mitochondrial-derived peptide is humanin, a 24 aminoacid peptifde encoded within the $16 \mathrm{~S}$ ribosomal RNA gene (Capt et al., 2016), this peptide has been proposed to produce strong cytoprotective actions against various stress and diseases (Lee et al., 2013a). Humanin has demonstrated protective capacity in age-related diseases such as T2DM and Alzheimer's disease (Mahboobi et al., 2014) and is able to restore mitochondrial function after oxidative injury in retinal pigment epithelial cells (Sreekumar et al., 2016). These peptides open a new field in the study of regulatory mechanisms involved in the regulation of mitochondrial homeostasis associated with ageing and bioenergetics.

\section{Mitochondrial dysfunction is associated with age-dependent fat accumulation.}

A considerable body of evidence demonstrate that mitochondrial dysfunction also plays an important role in obesity and obesity-related diseases such as insulin resistance and fatty liver. Impaired mitochondrial function and dynamics have been associated with diet induced obesity mainly affecting liver and skeletal muscle (Putti et al., 2015). These dysfunctions have been modulated by CR and exercise (Putti et al., 2015). Further, induction of mitochondrial dysfunction by blocking respiration or knocking down the mitochondrial transcription factor $A$ induce insulin resistance in human adipocytes and affects the secretion of adiponectin in an effect attributed to the increase in ROS production by defective mitochondria (Wang et al., 2013).

Regulators of mitochondrial activity and turnover such as sirtuins have been associated with the biogenesis of adipocytes. Very recently, a key role of SIRT1 
in hyperplasia, the increase of cell number, of adipocytes, has been suggested. Interestingly, hyperplasia has been also associated with dysregulation of metabolism and enhanced differentiation in adipocytes (Abdesselem et al., 2016). This indicates that SIRT1 is needed for a correct regulation of the adipose tissue function (Abdesselem et al., 2016).

In relationship with mitochondrial dynamics, changes in the mitochondrial network have been associated with obesity-related diseases. Apart of the effects due to defects in Mfn1 and 2 found in neurons, skeletal muscle or monocytes, a screen of differentially expressed genes from skeletal muscle of obese rats has identified Mfn2 as a suppressor of obesity (Bach et al., 2003). Further, conditions that increase energy expenditure such as cold exposure, chronic exercise or nutraceuticals as resveratrol are associated with higher Mfn2 expression in skeletal muscle and brown adipose tissue in mice and humans (Cartoni et al., 2005; Dolinsky et al., 2013; Soriano et al., 2006). Interestingly, these conditions are known to induce SIRT1 that has been recently associated with the suppression of mitochondrial dysfunction in a mouse model of ischemia by modulating Mfn2 activity by deacetylation indicating a connection between SIRT1 and mitochondrial dynamics (Biel et al., 2016). On the other hand, higher mitochondrial fission has been also associated with fat accumulation in genetically induced obesity (ob/ob) mice or in high-fat induced obesity (Jheng et al., 2012) and with insulin resistance in the same mice model (Holmstrom et al., 2012).

Prolongevity $\mathrm{CR}$ mimetics such as rapamycin and metformin have also been proposed to modulate mitochondrial dynamics in obesity and age-related diseases (Mercken et al., 2012). Although rapamycin has been associated with 
the prevention of age-related diseases and the extension of lifespan in both, obese and lean mice (Liu et al., 2014) and to avoid hyperlipidemia and diabetes-like syndrome in aged obese rats (Scarpace et al., 2016), a recent paper indicate that rapamycin reduces lifespan in the obese $\mathrm{db} / \mathrm{db}$ mice (Sataranatarajan et al., 2016). In the case of metformin, it has been associated with the prevention of sedentarism-dependent damages (Senesi et al., 2016). Further, metformin-dependent AMPK activation has recently demonstrated protection of mitochondria in huntington's disease by affecting mitochondrial dynamics (Jin et al., 2016). These results demonstrate that these pharmaceuticals compouds are promising in modulation of mitochondrial activity in ageing and obesity although more studies are needed to demonstrate their effectivity in mammals and humans.

In T2DM and obese people abnormalities due to lower mitochondrial functionality have been shown. Interestingly, in these cases a transient increase in oxidative capacity was found in human liver at the same time that oxidative stress increases (Koliaki and Roden, 2016). These changes have been associated with the increase in mitochondrial fragmentation and down regulation of fusion-regulatory factors associated with impaired bioenergetics in human muscle (Bach et al., 2005). Reduced expression of Mfn2 has been found in skeletal muscle in both, obese and T2DM patients (Bach et al., 2005). Lower levels of Mfn2 have been associated with the fragmentation of the mitochondrial network and the decrease of membrane potential in rat skeletal muscle (Pich et al., 2005). This reduction is directly proportional to BMI and inversely proportional to insulin response (Pich et al., 2005). Mfn2 depleted rat muscle cells show a reduced proton leak and lower bioenergetics efficiency 
indicated by lower glucose oxidation and mitochondrial respiration (Bach et al., 2003). This lower bioenergetics efficiency due to Mfn2 loss of function has been associated with the development of obesity by reducing energy expenditure and increasing fat energy store in mammals and humans (Liesa et al., 2009). On the other hand, physical exercise increases Mfn1 and 2 in human skeletal muscle indicating the importance of mitochondrial dynamics in the bioenergetic response of mitochondria to energy requirements (Cartoni et al., 2005).

Prohibitin has been recently linked to the regulation of mitochondrial activity and the development of obesity. Prohibitin is a complex of two proteins, PHB-1 and PHB-2, that has been associated with longevity in studies carried out in worms (Lourenco et al., 2015). Prohibitins are ubiquitous, evolutionary conserved proteins that form a ring-like complex at the inner membrane of the mitochondria (Artal-Sanz and Tavernarakis, 2009). They have been associated with mitochondrial fusion by stabilizing OPA1 that mediates inner membrane fusion and cristae morphogenesis. However, the exact function of this complex in mitochondria is not completely clear although it has been associated with mitochondrial function, fat metabolism and oxidative stress response in mice (Theiss et al., 2009). Interestingly, overexpression of prohibitin in adipocytes produces the development of obesity in mice accompanied by the induction of mitochondrial biogenesis (Ande et al., 2014). This effect contrasts with the higher mitochondrial fission associated with fat accumulation in obese mice (Jheng et al., 2012). On the other hand, downregulation of PHB by microRNA$27 \mathrm{a}$ and $27 \mathrm{~b}$ resulted in impaired mitochondrial biogenesis and structure integrity, lower activity of complex I and higher ROS production in adiposederived stem cells (Kang et al., 2013). Moreover, inactivation of PHB-2 in mice 
brain affects mitochondrial structure and activity resulting in neurodegeneration by hyperphosphorylation of Tau (Merkwirth et al., 2012) or alters beta-cell function in pancreas producing diabetes (Supale et al., 2013). All these facts highlight the importance of a balanced mitochondrial dynamics in the physiology adipose and other tissues and the complex regulation of the different factors involved in mitochondrial fusion and fission. Further studies are needed to understand how this balance is controlled and how ageing can affect it producing a cascade of events leading to the age-related diseases associated with fat accumulation.

Regarding sestrins, transgenic mice deficient in Sens2 and Sens3 genes have demonstrated the important role of these proteins in the suppression of age and obesity-associated mitochondrial disorders (Bae et al., 2013; Lee et al., 2012). Sesn2 KO mice show glucose intolerance, lower insulin response and development of hepatosteatosis (Lee et al., 2013b; Lee et al., 2012). On the other hand, Sens3 has been recently associated with the activation of mTORC2 and Akt phosphorylation enhancing insulin sensitivity and glucose metabolism in mice (Tao et al., 2015). It has been suggested that hypernutrition and lack of exercise can induce chronic mTORC1 activation that is accompanied by Sesn2 up-regulation in order to control the excessive activity of mTORC1 (Lee et al., 2010). Other studies carried out in rats proposed that sestrins-dependent mTORC1 inactivation through AMPK induction is critical in the maintenance of autophagy and, then, the elimination of dysfunctional mitochondria (Ishihara et al., 2013). Interestingly, sestrins are also regulated by oxidative stress through p53, NRF-2, AP-1 and FOXO and they regulate several components that reduce ROS production, increase mitochondrial biogenesis and induce 
mitophagy increasing the efficiency of oxidative metabolism in mice (Lee et al., 2013b).

All these evidences suggest that sestrins could be considered key factors in the control of mitochondrial homeostasis in ageing and obesity and probably T2DM. Research performed in invertebrates demonstrate that the inactivation of sestrins results in metabolic pathologies that include oxidative damage, mitochondrial dysfunction and fat accumulation (Lee et al., 2013b). However, to date there is no information available about the activity of sestrins in adipose tissue and its behaviour during ageing. Indirect evidences indicate a putative role of this family of proteins in fat accumulation. Resveratrol (RSV), a polyphenol that prevents lipid accumulation in liver, blocks Liver $\mathrm{X}$ receptor (LXRa)-mediated hepatic lipogenesis by inducing Sens2 gene (Jin et al., 2013), indicating a putative role of this family of proteins in fat accumulation. $L X R \alpha$ is a transcription factor that modulates lipid synthesis in liver (Joseph et al., 2002) and regulates insulin-stimulated lipogenesis (Chen et al., 2004). LXRa induces the expression of fatty acid synthesis (FAS), acetyl-CoA carboxylase (ACC) and sterol regulatory element binding protein 1c (SREBP-1c) (Grefhorst et al., 2002; Repa et al., 2000). Therefore, the Sens2-dependent repression of LXRa induced by RSV would indicate a key role of these proteins in fat accumulation during ageing.

Do sestrins affect fat accumulation and modulate mitochondrial activity and turnover during ageing? No information is available to date. However, the above indicated evidences point to these proteins as putative key regulators. In fact, although no studies have been performed yet in humans, the activity of sestrins 
has been recently suggested in the effect of exercise to prevent sarcopenia, frailty and obesity in the elderly (Sanchis-Gomar, 2013).

All these reports indicate that there is a downregulation of fusion in mitochondria in obesity related diseases. When fat accumulates, mitochondrial activity can decrease by increasing fission processes and accumulating then, low potential small mitochondria. This response is opposite to the behaviour found during ageing in which mitochondrial network tends to increase by inducing fusion in a response that probably is triggered to maintain the capacity of mitochondria (Fig. 2). It is probable that the incapacity to control mitochondrial dynamics during ageing, when fat accumulates in WAT, liver or muscle, produces the accumulation of damaged mitochondria and the resulting consequences as metabolism unbalance, insulin resistance or oxidative stress.

\section{Metabolic syndrome, fat accumulation and mitochondria.}

Obesity and T2DM are considered age-related diseases. In several of the models studied including rodents and humans, these diseases are associated with mitochondrial dysfunction indicated by low expression of mtDNA and reduction of the levels of proteins involved in oxidative phosphorylation (OXPHOS). Thus, mitochondrial dysfunction has been described in the main organs involved in the development of metabolic syndrome during ageing such as muscle, liver and adipose tissue (Patti and Corvera, 2010). 
The increase in fat mass is one of the major determinants of insulin resistance (Larson-Meyer et al., 2006). In humans, it has been shown that insulin resistance is responsible of the increase of lipids in liver and skeletal muscle and the decrease of the capacity of adipose tissue to store lipids (Toledo and Goodpaster, 2013). On the other hand, deficiency in mitochondrial activity in muscle is associated with obesity and T2DM. These mitochondrial deficiencies include reduction of fatty acid oxidation, lower expression of genes involved in oxidative activity and decrease of electron transport chain activities (Mootha et al., 2003; Patti and Corvera, 2010; Simoneau et al., 1999; Toledo and Goodpaster, 2013). Further, alterations in mitochondrial dynamics and in particular in Mfn2, have been associated with a reduced mitochondrial function in skeletal muscle in obesity and T2DM (Zorzano et al., 2009) and recently aberrant mitochondrial fission has been also associated with insulin resistance in mouse skeletal muscle (Jheng et al., 2012). The deficiency in the capacity of mitochondria to metabolize lipids has been proposed to predispose WAT and skeletal muscle to accumulate lipids and then, to the development of insulin resistance (Lowell and Shulman, 2005). However, other studies performed in rats suggested that insulin resistance is accompanied by an increase in mitochondrial content and an increase in oxidation of lipids (Dela and Helge, 2013; Garcia-Roves et al., 2007; Hancock et al., 2008; Turner et al., 2007). These discrepancies indicate that the role of mitochondria in muscle in high-fat diet induced T2DM is not completely clear. Probably, the accumulation of fat in muscle induces a higher oxidative metabolism based on fatty acids catabolism but this can be accompanied by the increase of mitochondria including damaged and ROS-producing structures that accumulate by a deficient 
mitochondrial turnover and fusion/fission mechanisms. In fact, it has been shown that, in both, rodents and humans, skeletal muscle from high-fat fed conditions show higher $\mathrm{H}_{2} \mathrm{O}_{2}$-mitochondrial production affecting the redox state of the cells but without changes in the mitochondrial respiratory function (Anderson et al., 2009). Further experiments performed in rats demonstrated that exposure to prepregnancy maternal obesity impairs the activity of factors involved in mitochondrial dynamics in the offspring (Borengasser et al., 2014). The accumulation of damaged mitochondria would explain the increase of ROS in skeletal muscle associated with insulin resistance of obese individuals and the appearance of T2DM and muscle malfunction during normal ageing as results of the deteriorated physiology of the organs (Barbieri et al., 2013). In fact, the elucidation of the mechanisms affecting mitochondrial dynamics in skeletal muscle from obese people has been suggested to be essential to understand muscle insulin resistance and to find therapeutic targets (Jheng et al., 2015).

On the other hand, obesity is associated with the impairment of the ability to transit between fatty acid and glucose metabolism. This impairment is known as "metabolic inflexibility" (Griffin et al., 2015), that is present in obese people since they are able to oxidize lipids in insulin-stimulated conditions instead to metabolize glucose being mitochondria the key organelles affecting this incapacity (Storlien et al., 2004).

Among the regulators of metabolism, AMPK seems to be the key component in the progression of metabolic syndrome and insulin resistance in WAT. Phosphorylated AMPK modulates the activity of many downstream substrates resulting in the increase in lipid oxidation and the uptake of glucose (Jager et 
al., 2007). Moreover, deficiency in AMPK results in glucose intolerance, lower physical capacity and obesity; symptoms of metabolic disease (Steinberg et al., 2010). Taken into consideration the central role of AMPK in the control of metabolism and mitochondria (Burkewitz et al., 2014), modification of levels or activity of AMPK could explain the deterioration of adipose tissue physiology during ageing. Regarding this aspect, a new regulator of AMPK, prolyl isomerase, Pin1, has been shown to rise in high fat state in mouse liver (Nakatsu et al., 2011). This increase is associated with the accumulation of fat since in Pin1 KO mice, a high fat diet-induced obesity resistant phenotype has been described (Nakatsu et al., 2015). Interestingly, the deregulation of Pin1 has been also implied in ageing and age-related diseases (Lee et al., 2011). Taken into consideration the plasticity of mitochondria and the complex relationship between the different regulators of mitochondrial dynamics and turnover, the study of the control of these regulators during ageing and fat overloading must be studied in deep to design efficient strategies against ageing-related diseases caused by deficient fat metabolism.

\section{Inflammation and lipid accumulation.}

Another important fact to be taken into consideration in the accumulation of fat during ageing is the proinflammatory status that accompanies obesity. It is known that visceral fat is an important source of proinflammatory cytokines contributing to insulin resistance. In this process, the activation of different forms of inflammasome is one of the key factors. Nod-like receptors (NLRs) are an evolutionary conserved family of cytoplasmic receptors whose function is to 
recognize pathogen- and danger-associated molecular patterns (PAMPs and DAMPs). These receptors form multimeric protein complexes that bind to a caspase activation and recruitment domain (CARD) and caspase 1 (Casp-1). After recognizing PAMPs or DAMPs, Casp-1 is activated and catalyses the transformation of proinflammatory cytokines to their mature forms (Lupfer and Kanneganti, 2012). The release of danger signals from mitochondria promotes the formation of inflammasomes. In fact, mitochondria is considered a central regulator of NLRP3 inflammasome activation through $\mathrm{ROS}, \mathrm{Ca}^{2+}$, decrease of $\mathrm{NAD}^{+}$, Mfn or mitochondrial DNA (Gurung et al., 2015). In patients with diabetes, the inhibition of autophagy results in the accumulation of dysfunctional mitochondria leading to higher ROS production and activating inflammasome (Harijith et al., 2014). On the other hand, Casp-1 activates several pathways that precipitate mitochondrial disassembly, increase ROS production and breaks up mitochondrial network, triggering a vicious cycle in which mitochondrial dysfunction activates inflammasome and Casp-1 induces mitochondrial dysfunction (Yu et al., 2014). Prolongevity effectors such as CR or resveratrol would reduce NLRP3-inflammasome activation preventing the activation of this vicious cycle. In fact, we have recently found that NLRP3 activity increases in mouse liver during ageing but this activity is inhibited by RSV probably by reducing the level of oxidative stress increasing the mitochondrial turnover and eliminating by this mechanism ROS-producing deficient mitochondria (Baur et al., 2006; Tung et al., 2015a).

Recently, a relationship between lipid accumulation in liver and inflammasome complex activity has been suggested. Lipogenesis in liver is modulated by sterol regulatory element binding proteins (SREBPs). These proteins are activated by 
hepatitis $\mathrm{C}$ virus and induce the accumulation of lipid droplets probably by activation of the NLRP3-inflammasome complex (McRae et al., 2015). Moreover, chronically overactive inflammasome activity and production of IL-1 $\beta$ has been associated with the abnormal lipid accumulation in old rat liver resulting in steatohepatitis (Chung et al., 2015). The activity of inflammasome has been associated with dysregulation of PPARa and SREBP1c (Chung et al., 2015). Furthermore, a NLRP3 KO mice model has demonstrated that the activity of this inflammasome is essential in the development of high-fat dietinduced obesity and in the development of insulin resistance (Stienstra et al., 2011). On the other hand, the increase of the levels of proinflammatory cytokines such as TNF- $\alpha$ and IL-6 found in obesity produce inhibitory effects on Mfn2 expression in human cultured cells (Bach et al., 2005) probably inducing a dysfunction in mitochondrial homeostasis.

These studies suggest that mitochondria can be on the origin of the overactivation of inflammasome in liver and other organs and then, accumulation of fat and development of steatohepatitis. Obesity is, at the same time, responsible of the maintenance of a chronic inflammatory status producing a vicious cycle. Furthermore, the inflammasome activation in fat tissues will trigger fat cell senescence, a phenomenon connected with the senescenceassociated secretory phenotype (SASP) (Newsholme and de Bittencourt, 2014). Then, fat accumulation would promote inflammasome associated SASP that would induce cellular senescence in other tissues of the organism aggravating by this mechanism the obesity-dependent chronic inflammation in another vicious cycle (Newsholme and de Bittencourt, 2014) (Fig.3). 


\section{Do fat accumulation and mitochondrial dysfunction depend on sexual}

hormones?

In humans, fat deposits depend on the gender of the individual (Palmer and Clegg, 2015). Men tend to accumulate visceral fat whereas women accrue more subcutaneous depots prior to menopause but, after menopause, fat depots in women shift to visceral depots (Palmer and Clegg, 2015). The cause of this difference resides in sexual hormones. Oestrogens and their receptors favour lipid accumulation in subcutaneous depot in women but visceral fat deposition in men. The differences in the metabolism of visceral and subcutaneous fat has been related to a higher risk of metabolic syndrome in men than in women due to a higher free fatty acid production in visceral fat than in subcutaneous fat (Palmer and Clegg, 2015). This will explain why women, with higher levels of subcutaneous fat, show less risk for diseases associated with obesity than men that show higher levels of visceral fat deposition. Visceral fat accumulation is associated with many age-related diseases (Huffman and Barzilai, 2009) accompanied by the accumulation of fat in tissues such as the heart, liver and skeletal muscle which increases risk for insulin resistance and cardiovascular disease (Kuk et al., 2009).

Some data indicate that oestrogens control the accumulation of fat during ageing. In premenopausal women, oestrogens protect against weight gain by increasing energy expenditure, whereas postmenopausal women gain weight by the natural decrease in endogenous estradiol levels (Gambacciani et al., 1997). These reductions can be prevented by oestrogen replacement therapy, indicating a direct relationship between the levels of these hormones and metabolic control and probably mitochondrial activity (Gambacciani et al., 
1997). In men, the decrease in testosterone levels can be responsible of the increase of adiposity and fat accumulation in non-adipose tissue during ageing (Mudali and Dobs, 2004). In a similar response that women, testosterone therapy prevents the gain in visceral fat in non-obese ageing men (Allan et al., 2008).

Another interesting paper has associated the accumulation of fat during ageing with higher levels of follicle-stimulating hormone (FSH) in both men and women (Liu et al., 2015). In this paper, authors indicate that the increase of this hormone not only is responsible for fat accumulation but also affects fat redistribution from subcutaneous and abdominal fat to visceral fat, a fact associated with ageing (Zamboni et al., 2005). The accumulation of fat in heart, liver and skeletal muscle during ageing has been also attributed to the $\mathrm{FSH}$ rise since skeletal muscle and liver also express FSH-receptor (Liu et al., 2015).

Changes in oestrogens levels can also modulate the genetic expression of mitochondrial factors and then, be also responsible of mitochondrial dysfunction effect in fat accumulation during ageing (Vasconsuelo et al., 2013). It has been proposed that estradiol and testosterone affect mitochondria during ageing through receptors located even in mitochondria. These oestrogens would directly regulate nuclear DNA-encoded proteins and mitochondrial transcription factors affecting by this mechanism mitochondrial biogenesis (Vasconsuelo et al., 2013). It has been also proposed that mitochondrial genome contains sequences that are similar to those of nuclear hormone-responsive elements (Sekeris, 1990). In fact, these elements have been found in mtDNA accompanied by oestrogen and androgen receptors (Demonacos et al., 1995; Demonacos et al., 1996). The appropriate receptors can interact with these 
sequences to induce hormone-dependent mitochondrial gene expression (Psarra et al., 2006). In agreement with this, oestrogens and androgens can affect the transcription of mitochondria OXPHOS components (Scheller et al., 2003). These hormone-responsible elements are also found in nuclear gene encoding mitochondrial transcription factors that also modulate OXPHOS gene expression (Psarra et al., 2006).

Changes in oestrogens during ageing can also affect directly the activity of regulators of mitochondria. In rats, a model of menopause by using ovariectomization suggested that reduction of oestrogens produced the decrease of PGC1 $1 \alpha$ expression and the reduction of $\beta$-oxidation in muscle (Cavalcanti-de-Albuquerque et al., 2014). This suggests that an oestrogendependent effect can induce accumulation of fat and probably insulin resistance in muscle.

Despite these reports, a question remains to be answered; can mitochondrial dysfunction be a response to the reduction of sexual hormones during ageing? Low levels of oestrogens decrease PGC1a expression indicating a putative decrease in mitochondrial biogenesis (Cavalcanti-de-Albuquerque et al., 2014). Further, mitochondrial dynamics are also regulated by these hormones. In the human breast cancer cell line MCF-7, the mRNA levels of mitochondrial fusion genes increase during estradiol treatment whereas FIS1 gene expression decreases (Sastre-Serra et al., 2012). In these cells, estradiol treatment also induced mitochondrial biogenesis (Sastre-Serra et al., 2012). The same effect was found in other breast cancer cell lines (Sastre-Serra et al., 2013). Further, in rat models, the expression of prohibitins responds to the levels of oestrogens in WAT. The level of prohibitins were stimulated by estradiol in WAT and liver in 
both, controls and high-fat fed rats (Choi et al., 2015). Studies performed in other tissues demonstrated that prohibitin genes are induced by oestrogen receptors (He et al., 2011). Taken into consideration the role of all these proteins in the fusion process in mitochondria, it is clear that modifications in the levels of sexual hormones during ageing will affect mitochondrial dynamics and probably affect the accumulation of damaged mitochondria in adipocytes and other fat-accumulating organs. The relationship between oestrogen levels, ageing and mitochondrial activity in fat must be further studied (Fig.4).

Mitochondrial function in POMC neurons and its effect on the control of satiety.

Satiety and hunger is controlled by neuropeptide-Y (NPY), agouti-related protein (Agrp) and pro-opiomelanocortin (POMC) neurons located in the arcuate nucleus of the hypothalamus. Both obesity and ageing are associated with rewiring of a principal brain pathway modulating energy homeostasis through promoting reduced activity of satiety pro-opiomelanocortin (POMC) neurons. It has been suggested that the activity of these neurons is importantly affected by mitochondrial dynamics (Nasrallah and Horvath, 2014). In studies performed in mice it has been demonstrated that mitochondrial fusion increases in orexigenic NPY/Agrp neurons inducing neuronal activity that control the storage of excess of energy in fat when mice are fed with high-fat diet (Dietrich et al., 2013). The activity of these neurons is impaired when the mitochondrial fusion mechanism is altered producing resistance to fat gain during high fat diet (Dietrich et al., 2013). However, the depletion of Mfn2 in the anorexigenic POMC neurons 
causes severe obesity and leptin resistance probably by affecting ER-stress (Schneeberger et al., 2013). Moreover, a dysregulation of the control of mitochondrial activity and turnover could be also associated with dysfunctions in the control of satiety. A recent paper indicates that mTOR activity is elevated in POMC neurons in aged mice. This increase produces cell hypertrophy that the authors associate with obesity-related deterioration of POMC neurons (Yang et al., 2012). On the contrary, the inhibition of mTOR in these neurons caused reduction of food intake and weight loss in old mice indicating the importance of this regulator in satiety control (Yang et al., 2012). These and other results suggest that mitochondrial dynamics in NPY/Agrp and POMC neurons also play an important role in the regulation of the energy balance and in diet and agerelated obesity.

\section{Concluding remarks.}

Many evidences indicate that mitochondrial dysfunction is associated with the development of obesity and the appearance and progression of age-related diseases (Fig.5). Regulators of metabolism such as mTOR, AMPK and sirtuins are involved in this effect and the complex equilibrium between their activities modulates mitochondrial biogenesis, dynamics and turnover. This regulation is affected by sexual hormones that change during ageing and probably the reduction of oestrogens is one of the key factors involved in mitochondrial dysfunction and fat accumulation in humans. During ageing a tendency to increase fusion in mitochondria has been reported in order to maintain mitochondrial activity; however, fat accumulation induces a higher activity of 
fission mechanisms promoting the accumulation of small and less efficient mitochondria. Probably these two opposite processes end in the accumulation of damaged mitochondria that increase oxidative damage that contribute to the appearance and impairment of insulin resistance, metabolic dysfunction and the release of proinflammatory mediators. In a vicious cycle, insulin resistance and proinflammatory cytokines promote more damage in mitochondria. The study of the response of these metabolic regulators to prolongevity effectors such as $\mathrm{CR}$, nutra- and pharmaceuticals or exercise and the role of new modulators such as prohibitins or sestrins in ageing progression will help us to develop therapeutic strategies to delay the damaging effect of fat accumulation during ageing.

\section{Funding.}

The research group is funded by the Andalusian Government grant BIO177 (FEDER funds of European Commission). Research has been funded by the Spanish Ministry of Economy and Competitiveness grant DEP2012-39985. Authors are also members of the CIBERER, Instituto Carlos III, of the Spanish Ministry of Health. 


\section{Figure legends.}

Graphical abstract. Mitochondrial homeostasis is basic to maintain a balanced metabolic activity in fat accumulating tissues including adipose tissue, muscle and liver. Ageing and the decay of oestrogens produce an unbalance in mitochondrial homeostasis causing the accumulation of damaged mitochondria as giant or small and damaged mitochondria. Decay of mito- and autophagy processes is also affected. These events produce the reduction of respiration, membrane potential and the increase of reactive oxygen species. Prolongevity factors such as calorie restriction, exercise or several nutraceutical such as polyphenols can reduce mitochondrial dysfunction by maintaining mitochondrial biogenesis, fusion/fission balance and mito- and autophagy during ageing preventing then fat accumulation.

Fig. 1. Molecular mechanisms involved in the maintenance of mitochondrial lipid metabolism. Two major systems are involved in the control of metabolism in cells: mTOR and AMPK/SIRT. High fat diets, fat accumulation of T2DM activates mTORC1 that reduces lipid consumption by affecting mitochondrial biogenesis, turnover and dynamics through inhibiting PGC1a. On the other hand, effectors such as CR, exercise of polyphenols activate AMPK/SIRT that inhibit mTORC1 and induce mitochondrial biogenesis through PGC1 $\alpha$ and mitophagy maintaining a mitochondrial balanced activity.

Fig. 2. Mitochondrial dynamics during fat accumulation and ageing. Balanced mitochondrial activity maintains a control of mitochondrial population by fusion/fission balance regulated by Mfn 1 and 2, OPA1 and prohibitins (fusion) and DRP1 and FIS1 (fission) cooperation and the biogenesis of the 
components of mitochondria. A dysfunction in these factors can cause the accumulation of giant mitochondria in ageing or small and damaged mitochondria that increase ROS production by decreasing fusion mechanisms and blocking autophagy processes. Regulation of these processes by AMPK and $\mathrm{mTOR} 1$ regulate these processes.

Fig 3. Role of the inflammasome in fat accumulation during ageing. Mitochondrial dysfunction produces NLRP3 inflammasome activation by increasing ROS that acts as Damage-activating molecular pattern. This activation is associated with cell senescence and SASP and the evolution of hepatosteatosis, obesity and T2DM.

Fig 4. Hormonal control of mitochondrial activity can affect fat accumulation. Age-dependent oestrogen decay will affect mitochondrial dynamics by decreasing mitochondrial fusion and PGC1a activity. This will produce mitochondrial dysfunction. At the same time, the increase of FSH found in aged individuals will contribute to the accumulation of visceral fat that, in a vicious cycle, will contribute to higher mitochondrial dysfunction by inhibiting mito- and autophagy through mTORC1 activity as bas been indicated in Fig. 2 . Visceral fat accumulation is associated with the appearance and impairment of T2DM, evolution of hepatosteatosis and obesity.

Fig. 5. Mitochondrial dysfunction is at the centre of the regulation of fat metabolism during ageing. The balanced activity of mitochondria is essential to control satiety and prevent the appearance and progression of T2DM, obesity and inflammation, hallmarks of age-dependent accumulation of fat. Ageing, high fat diet and sexual hormones decay causes mitochondrial dysfunction whereas 
prolongevity interventions such as calorie restriction, exercise and diet rich in nutraceuticals prevent mitochondrial decay and improve the progression of ageing and fat accumulation during ageing. 


\section{References.}

Abdesselem, H., Madani, A., Hani, A., Al-Noubi, M., Goswami, N., Ben Hamidane, H., Billing, A.M., Pasquier, J., Bonkowski, M.S., Halabi, N., Dalloul, R., Sheriff, M.Z., Mesaeli, N., EIRayess, M., Sinclair, D.A., Graumann, J., Mazloum, N.A., 2016. SIRT1 Limits Adipocyte Hyperplasia through c-Myc Inhibition. J Biol Chem 291, 2119-2135. Doi: 10.1074/jbc.M115.675645.

Abranches, M.V., Oliveira, F.C., Conceicao, L.L., Peluzio, M.D., 2015. Obesity and diabetes: the link between adipose tissue dysfunction and glucose homeostasis. Nutr Res Rev 28, 121-132. Doi: 10.1017/S0954422415000098.

Alers, S., Loffler, A.S., Wesselborg, S., Stork, B., 2012. Role of AMPK-mTORUlk1/2 in the regulation of autophagy: cross talk, shortcuts, and feedbacks. Mol Cell Biol 32, 2-11. Doi: 10.1128/MCB.06159-11.

Allan, C.A., Strauss, B.J., Burger, H.G., Forbes, E.A., McLachlan, R.I., 2008. Testosterone therapy prevents gain in visceral adipose tissue and loss of skeletal muscle in nonobese aging men. J Clin Endocrinol Metab 93, 139-146. Doi: 10.1210/jc.2007-1291.

Ande, S.R., Nguyen, K.H., Padilla-Meier, G.P., Wahida, W., Nyomba, B.L., Mishra, S., 2014. Prohibitin overexpression in adipocytes induces mitochondrial biogenesis, leads to obesity development, and affects glucose homeostasis in a sex-specific manner. Diabetes 63, 3734-3741. Doi: 10.2337/db13-1807.

Anderson, E.J., Lustig, M.E., Boyle, K.E., Woodlief, T.L., Kane, D.A., Lin, C.T., Price, J.W., 3rd, Kang, L., Rabinovitch, P.S., Szeto, H.H., Houmard, J.A., Cortright, R.N., Wasserman, D.H., Neufer, P.D., 2009. Mitochondrial H2O2 emission and cellular redox state link excess fat intake to insulin resistance in both rodents and humans. J Clin Invest 119, 573-581. Doi: 10.1172/JCI37048. 
Artal-Sanz, M., Tavernarakis, N., 2009. Prohibitin couples diapause signalling to mitochondrial metabolism during ageing in C. elegans. Nature 461, 793-797. Doi: $10.1038 /$ nature08466.

Bach, D., Naon, D., Pich, S., Soriano, F.X., Vega, N., Rieusset, J., Laville, M., Guillet, C., Boirie, Y., Wallberg-Henriksson, H., Manco, M., Calvani, M., Castagneto, M., Palacin, M., Mingrone, G., Zierath, J.R., Vidal, H., Zorzano, A., 2005. Expression of Mfn2, the Charcot-Marie-Tooth neuropathy type 2A gene, in human skeletal muscle: effects of type 2 diabetes, obesity, weight loss, and the regulatory role of tumor necrosis factor alpha and interleukin-6. Diabetes 54, 2685-2693. Doi.

Bach, D., Pich, S., Soriano, F.X., Vega, N., Baumgartner, B., Oriola, J., Daugaard, J.R., Lloberas, J., Camps, M., Zierath, J.R., Rabasa-Lhoret, R., Wallberg-Henriksson, H., Laville, M., Palacin, M., Vidal, H., Rivera, F., Brand, M., Zorzano, A., 2003. Mitofusin-2 determines mitochondrial network architecture and mitochondrial metabolism. A novel regulatory mechanism altered in obesity. J Biol Chem 278, 17190-17197. Doi: 10.1074/jbc.M212754200.

Bae, S.H., Sung, S.H., Oh, S.Y., Lim, J.M., Lee, S.K., Park, Y.N., Lee, H.E., Kang, D., Rhee, S.G., 2013. Sestrins activate Nrf2 by promoting p62-dependent autophagic degradation of Keap1 and prevent oxidative liver damage. Cell Metab 17, 73-84. Doi: 10.1016/j.cmet.2012.12.002.

Barbieri, E., Sestili, P., Vallorani, L., Guescini, M., Calcabrini, C., Gioacchini, A.M., Annibalini, G., Lucertini, F., Piccoli, G., Stocchi, V., 2013. Mitohormesis in muscle cells: a morphological, molecular, and proteomic approach. Muscles Ligaments Tendons J 3, 254-266. Doi. 
Baur, J.A., Chen, D., Chini, E.N., Chua, K., Cohen, H.Y., de Cabo, R., Deng, C., Dimmeler, S., Gius, D., Guarente, L.P., Helfand, S.L., Imai, S., Itoh, H., Kadowaki, T., Koya, D., Leeuwenburgh, C., McBurney, M., Nabeshima, Y., Neri, C., Oberdoerffer, P., Pestell, R.G., Rogina, B., Sadoshima, J., Sartorelli, V., Serrano, M., Sinclair, D.A., Steegborn, C., Tatar, M., Tissenbaum, H.A., Tong, Q., Tsubota, K., Vaquero, A., Verdin, E., 2010. Dietary restriction: standing up for sirtuins. Science 329, 1012-1013; author reply 1013-1014. Doi: 10.1126/science.329.5995.1012.

Baur, J.A., Pearson, K.J., Price, N.L., Jamieson, H.A., Lerin, C., Kalra, A., Prabhu, V.V., Allard, J.S., Lopez-Lluch, G., Lewis, K., Pistell, P.J., Poosala, S., Becker, K.G., Boss, O., Gwinn, D., Wang, M., Ramaswamy, S., Fishbein, K.W., Spencer, R.G., Lakatta, E.G., Le Couteur, D., Shaw, R.J., Navas, P., Puigserver, P., Ingram, D.K., de Cabo, R., Sinclair, D.A., 2006. Resveratrol improves health and survival of mice on a high-calorie diet. Nature 444, 337342. Doi: 10.1038/nature05354.

Benigni, A., Perico, L., Macconi, D., 2016. Mitochondrial Dynamics Is Linked to Longevity and Protects from End-Organ Injury: The Emerging Role of Sirtuin 3. Antioxid Redox Signal. Doi: 10.1089/ars.2016.6682.

Benkhalifa, M., Ferreira, Y.J., Chahine, H., Louanjli, N., Miron, P., Merviel, P., Copin, H., 2014. Mitochondria: participation to infertility as source of energy and cause of senescence. Int $J$ Biochem Cell Biol 55, 60-64. Doi: 10.1016/j.biocel.2014.08.011.

Beregi, E., Regius, O., 1987. Comparative morphological study of age related mitochondrial changes of the lymphocytes and skeletal muscle cells. Acta Morphol Hung 35, 219-224. Doi. 
Biala, A.K., Dhingra, R., Kirshenbaum, L.A., 2015. Mitochondrial dynamics: Orchestrating the journey to advanced age. J Mol Cell Cardiol 83, 37-43. Doi: 10.1016/j.yjmcc.2015.04.015.

Biel, T.G., Lee, S., Flores-Toro, J.A., Dean, J.W., Go, K.L., Lee, M.H., Law, B.K., Law, M.E., Dunn, W.A., Jr., Zendejas, I., Behrns, K.E., Kim, J.S., 2016. Sirtuin 1 suppresses mitochondrial dysfunction of ischemic mouse livers in a mitofusin 2-dependent manner. Cell Death Differ 23, 279-290. Doi: 10.1038/cdd.2015.96.

Borengasser, S.J., Faske, J., Kang, P., Blackburn, M.L., Badger, T.M., Shankar, K., 2014. In utero exposure to prepregnancy maternal obesity and postweaning high-fat diet impair regulators of mitochondrial dynamics in rat placenta and offspring. Physiol Genomics 46, 841-850. Doi: 10.1152/physiolgenomics.00059.2014.

Brand, M.D., Nicholls, D.G., 2011. Assessing mitochondrial dysfunction in cells. Biochem J 435, 297-312. Doi: 10.1042/BJ20110162.

Budanov, A.V., Karin, M., 2008. p53 target genes sestrin1 and sestrin2 connect genotoxic stress and mTOR signaling. Cell 134, 451-460. Doi: 10.1016/j.cell.2008.06.028.

Burkewitz, K., Zhang, Y., Mair, W.B., 2014. AMPK at the nexus of energetics and aging. Cell Metab 20, 10-25. Doi: 10.1016/j.cmet.2014.03.002.

Capt, C., Passamonti, M., Breton, S., 2016. The human mitochondrial genome may code for more than 13 proteins. Mitochondrial DNA A DNA Mapp Seq Anal 27, 3098-3101. Doi: 10.3109/19401736.2014.1003924.

Cartoni, R., Leger, B., Hock, M.B., Praz, M., Crettenand, A., Pich, S., Ziltener, J.L., Luthi, F., Deriaz, O., Zorzano, A., Gobelet, C., Kralli, A., Russell, A.P., 
2005. Mitofusins $1 / 2$ and ERRalpha expression are increased in human skeletal muscle after physical exercise. J Physiol 567, 349-358. Doi: 10.1113/jphysiol.2005.092031.

Cartwright, M.J., Tchkonia, T., Kirkland, J.L., 2007. Aging in adipocytes: potential impact of inherent, depot-specific mechanisms. Exp Gerontol 42, 463471. Doi: 10.1016/j.exger.2007.03.003.

Cavalcanti-de-Albuquerque, J.P., Salvador, I.C., Martins, E.L., JardimMesseder, D., Werneck-de-Castro, J.P., Galina, A., Carvalho, D.P., 2014. Role of estrogen on skeletal muscle mitochondrial function in ovariectomized rats: a time course study in different fiber types. J Appl Physiol (1985) 116, 779-789. Doi: 10.1152/japplphysiol.00121.2013.

Civitarese, A.E., Carling, S., Heilbronn, L.K., Hulver, M.H., Ukropcova, B., Deutsch, W.A., Smith, S.R., Ravussin, E., Team, C.P., 2007. Calorie restriction increases muscle mitochondrial biogenesis in healthy humans. PLoS Med 4, e76. Doi: 10.1371/journal.pmed.0040076.

Collins, T.J., Berridge, M.J., Lipp, P., Bootman, M.D., 2002. Mitochondria are morphologically and functionally heterogeneous within cells. EMBO J 21, 16161627. Doi: 10.1093/emboj/21.7.1616.

Conley, K.E., Jubrias, S.A., Esselman, P.C., 2000. Oxidative capacity and ageing in human muscle. J Physiol 526 Pt 1, 203-210. Doi.

Chan, E.Y., 2009. mTORC1 phosphorylates the ULK1-mAtg13-FIP200 autophagy regulatory complex. Sci Signal 2, pe51. Doi: 10.1126/scisignal.284pe51.

Chen, G., Liang, G., Ou, J., Goldstein, J.L., Brown, M.S., 2004. Central role for liver $\mathrm{X}$ receptor in insulin-mediated activation of Srebp-1c transcription and 
stimulation of fatty acid synthesis in liver. Proc Natl Acad Sci U S A 101, 1124511250. Doi: 10.1073/pnas.0404297101.

Chen, H., Chan, D.C., 2005. Emerging functions of mammalian mitochondrial fusion and fission. Hum Mol Genet 14 Spec No. 2, R283-289. Doi: 10.1093/hmg/ddi270.

Chen, H., Chomyn, A., Chan, D.C., 2005. Disruption of fusion results in mitochondrial heterogeneity and dysfunction. J Biol Chem 280, 26185-26192. Doi: $10.1074 / j b c . M 503062200$.

Chistiakov, D.A., Sobenin, I.A., Revin, V.V., Orekhov, A.N., Bobryshev, Y.V., 2014. Mitochondrial aging and age-related dysfunction of mitochondria. Biomed Res Int 2014, 238463. Doi: 10.1155/2014/238463.

Choi, M., Chaudhari, H.N., Ji, Y.R., Ryoo, Z.Y., Kim, S.W., Yun, J.W., 2015. Effect of estrogen on expression of prohibitin in white adipose tissue and liver of diet-induced obese rats. Mol Cell Biochem 407, 181-196. Doi: 10.1007/s11010015-2468-1.

Chung, K.W., Lee, E.K., Kim, D.H., An, H.J., Kim, N.D., Im, D.S., Lee, J., Yu, B.P., Chung, H.Y., 2015. Age-related sensitivity to endotoxin-induced liver inflammation: Implication of inflammasome/IL-1beta for steatohepatitis. Aging Cell 14, 524-533. Doi: 10.1111/acel.12305.

de Brito, O.M., Scorrano, L., 2008. Mitofusin 2 tethers endoplasmic reticulum to mitochondria. Nature 456, 605-610. Doi: 10.1038/nature07534.

de Brito, O.M., Scorrano, L., 2009. Mitofusin-2 regulates mitochondrial and endoplasmic reticulum morphology and tethering: the role of Ras. Mitochondrion 9, 222-226. Doi: 10.1016/j.mito.2009.02.005. 
Dela, F., Helge, J.W., 2013. Insulin resistance and mitochondrial function in skeletal muscle. Int $\mathrm{J}$ Biochem Cell Biol 45, 11-15. Doi: 10.1016/j.biocel.2012.09.019.

Demonacos, C., Djordjevic-Markovic, R., Tsawdaroglou, N., Sekeris, C.E., 1995. The mitochondrion as a primary site of action of glucocorticoids: the interaction of the glucocorticoid receptor with mitochondrial DNA sequences showing partial similarity to the nuclear glucocorticoid responsive elements. J Steroid Biochem Mol Biol 55, 43-55. Doi.

Demonacos, C.V., Karayanni, N., Hatzoglou, E., Tsiriyiotis, C., Spandidos, D.A., Sekeris, C.E., 1996. Mitochondrial genes as sites of primary action of steroid hormones. Steroids 61, 226-232. Doi.

Dietrich, M.O., Liu, Z.W., Horvath, T.L., 2013. Mitochondrial dynamics controlled by mitofusins regulate Agrp neuronal activity and diet-induced obesity. Cell 155, 188-199. Doi: 10.1016/j.cell.2013.09.004.

Dolinsky, V.W., Rogan, K.J., Sung, M.M., Zordoky, B.N., Haykowsky, M.J., Young, M.E., Jones, L.W., Dyck, J.R., 2013. Both aerobic exercise and resveratrol supplementation attenuate doxorubicin-induced cardiac injury in mice. Am J Physiol Endocrinol Metab 305, E243-253. Doi: 10.1152/ajpendo.00044.2013.

Duvel, K., Yecies, J.L., Menon, S., Raman, P., Lipovsky, A.I., Souza, A.L., Triantafellow, E., Ma, Q., Gorski, R., Cleaver, S., Vander Heiden, M.G., MacKeigan, J.P., Finan, P.M., Clish, C.B., Murphy, L.O., Manning, B.D., 2010. Activation of a metabolic gene regulatory network downstream of mTOR complex 1. Mol Cell 39, 171-183. Doi: 10.1016/j.molcel.2010.06.022. 
Fabbri, E., Chia, C.W., Spencer, R.G., Fishbein, K.W., Reiter, D.A., Cameron, D., Zane, A.C., Moore, Z.A., Gonzalez-Freire, M., Zoli, M., Studenski, S.A., Kalyani, R.R., Egan, J.M., Ferrucci, L., 2016. Insulin Resistance is Associated with Reduced Mitochondrial Oxidative Capacity Measured by 31P Magnetic Resonance Spectroscopy in Non-Diabetic Participants from the Baltimore Longitudinal Study of Aging. Diabetes. Doi: 10.2337/db16-0754.

Feige, J.N., Lagouge, M., Auwerx, J., 2008. Dietary manipulation of mouse metabolism. Curr Protoc Mol Biol Chapter 29, Unit 29B 25. Doi: 10.1002/0471142727.mb29b05s84.

Fuku, N., Pareja-Galeano, H., Zempo, H., Alis, R., Arai, Y., Lucia, A., Hirose, N., 2015. The mitochondrial-derived peptide MOTS-c: a player in exceptional longevity? Aging Cell 14, 921-923. Doi: 10.1111/acel.12389.

Gambacciani, M., Ciaponi, M., Cappagli, B., Piaggesi, L., De Simone, L., Orlandi, R., Genazzani, A.R., 1997. Body weight, body fat distribution, and hormonal replacement therapy in early postmenopausal women. J Clin Endocrinol Metab 82, 414-417. Doi: 10.1210/jcem.82.2.3735.

Garcia-Roves, P., Huss, J.M., Han, D.H., Hancock, C.R., Iglesias-Gutierrez, E., Chen, M., Holloszy, J.O., 2007. Raising plasma fatty acid concentration induces increased biogenesis of mitochondria in skeletal muscle. Proc Natl Acad Sci U S A 104, 10709-10713. Doi: 10.1073/pnas.0704024104.

Genova, M.L., Lenaz, G., 2015. The Interplay Between Respiratory Supercomplexes and ROS in Aging. Antioxid Redox Signal 23, 208-238. Doi: 10.1089/ars.2014.6214. 
Gomes, L.C., Scorrano, L., 2008. High levels of Fis1, a pro-fission mitochondrial protein, trigger autophagy. Biochim Biophys Acta 1777, 860-866. Doi: 10.1016/j.bbabio.2008.05.442.

Gomes, L.C., Scorrano, L., 2011. Mitochondrial elongation during autophagy: a stereotypical response to survive in difficult times. Autophagy 7, 1251-1253. Doi: 10.4161/auto.7.10.16771.

Goncalves, R.L., Quinlan, C.L., Perevoshchikova, I.V., Hey-Mogensen, M., Brand, M.D., 2015. Sites of superoxide and hydrogen peroxide production by muscle mitochondria assessed ex vivo under conditions mimicking rest and exercise. J Biol Chem 290, 209-227. Doi: 10.1074/jbc.M114.619072.

Gonzalez-Freire, M., Cabo, R.d., Bernier, M., Sollott, S.J., Fabbri, E., Navas, P., Ferrucci, L., 2015. Reconsidering the Role of Mitochondria in Aging. The Journals of Gerontology Series A: Biological Sciences and Medical Sciences Doi: 10.1093/gerona/glv070. Doi: 10.1093/gerona/glv070.

Goto, T., Takano, M., 2009. Transcriptional role of FOXO1 in drug resistance through antioxidant defense systems. Adv Exp Med Biol 665, 171-179. Doi.

Gouspillou, G., Sgarioto, N., Norris, B., Barbat-Artigas, S., Aubertin-Leheudre, M., Morais, J.A., Burelle, Y., Taivassalo, T., Hepple, R.T., 2014. The relationship between muscle fiber type-specific PGC-1alpha content and mitochondrial content varies between rodent models and humans. PLoS One 9, e103044. Doi: 10.1371/journal.pone.0103044.

Gram, M., Vigelso, A., Yokota, T., Helge, J.W., Dela, F., Hey-Mogensen, M., 2015. Skeletal muscle mitochondrial H2 O2 emission increases with immobilization and decreases after aerobic training in young and older men. $\mathrm{J}$ Physiol 593, 4011-4027. Doi: 10.1113/JP270211. 
Grefhorst, A., Elzinga, B.M., Voshol, P.J., Plosch, T., Kok, T., Bloks, V.W., van der Sluijs, F.H., Havekes, L.M., Romijn, J.A., Verkade, H.J., Kuipers, F., 2002. Stimulation of lipogenesis by pharmacological activation of the liver $\mathrm{X}$ receptor leads to production of large, triglyceride-rich very low density lipoprotein particles. J Biol Chem 277, 34182-34190. Doi: 10.1074/jbc.M204887200.

Griffin, T.M., Humphries, K.M., Kinter, M., Lim, H.Y., Szweda, L.I., 2015. Nutrient sensing and utilization: Getting to the heart of metabolic flexibility. Biochimie. Doi: 10.1016/j.biochi.2015.10.013.

Guarente, L., 2011. Sirtuins, aging, and metabolism. Cold Spring Harb Symp Quant Biol 76, 81-90. Doi: 10.1101/sqb.2011.76.010629.

Guarente, L., 2013. Calorie restriction and sirtuins revisited. Genes Dev 27, 2072-2085. Doi: 10.1101/gad.227439.113.

Gurung, P., Lukens, J.R., Kanneganti, T.D., 2015. Mitochondria: diversity in the regulation of the NLRP3 inflammasome. Trends Mol Med 21, 193-201. Doi: 10.1016/j.molmed.2014.11.008.

Gwinn, D.M., Shackelford, D.B., Egan, D.F., Mihaylova, M.M., Mery, A., Vasquez, D.S., Turk, B.E., Shaw, R.J., 2008. AMPK phosphorylation of raptor mediates a metabolic checkpoint. Mol Cell 30, 214-226. Doi: 10.1016/j.molcel.2008.03.003.

Hancock, C.R., Han, D.H., Chen, M., Terada, S., Yasuda, T., Wright, D.C., Holloszy, J.O., 2008. High-fat diets cause insulin resistance despite an increase in muscle mitochondria. Proc Natl Acad Sci U S A 105, 7815-7820. Doi: 10.1073/pnas.0802057105. 
Handschin, C., Spiegelman, B.M., 2006. Peroxisome proliferator-activated receptor gamma coactivator 1 coactivators, energy homeostasis, and metabolism. Endocr Rev 27, 728-735. Doi: 10.1210/er.2006-0037.

Hardie, D.G., 2011. Sensing of energy and nutrients by AMP-activated protein kinase. Am J Clin Nutr 93, 891S-896. Doi: 10.3945/ajcn.110.001925.

Hardie, D.G., Ross, F.A., Hawley, S.A., 2012. AMPK: a nutrient and energy sensor that maintains energy homeostasis. Nat Rev Mol Cell Biol 13, 251-262. Doi: $10.1038 / \mathrm{nrm} 3311$.

Harijith, A., Ebenezer, D.L., Natarajan, V., 2014. Reactive oxygen species at the crossroads of inflammasome and inflammation. Front Physiol 5, 352. Doi: 10.3389/fphys.2014.00352.

He, B., Kim, T.H., Kommagani, R., Feng, Q., Lanz, R.B., Jeong, J.W., DeMayo, F.J., Katzenellenbogen, B.S., Lydon, J.P., O'Malley, B.W., 2011. Estrogenregulated prohibitin is required for mouse uterine development and adult function. Endocrinology 152, 1047-1056. Doi: 10.1210/en.2010-0732.

Holmstrom, M.H., Iglesias-Gutierrez, E., Zierath, J.R., Garcia-Roves, P.M., 2012. Tissue-specific control of mitochondrial respiration in obesity-related insulin resistance and diabetes. Am J Physiol Endocrinol Metab 302, E731-739. Doi: 10.1152/ajpendo.00159.2011.

Hou, Y.S., Guan, J.J., Xu, H.D., Wu, F., Sheng, R., Qin, Z.H., 2015. Sestrin2 Protects Dopaminergic Cells against Rotenone Toxicity through AMPKDependent Autophagy Activation. Mol Cell Biol 35, 2740-2751. Doi: 10.1128/MCB.00285-15.

Huffman, D.M., Barzilai, N., 2009. Role of visceral adipose tissue in aging. Biochim Biophys Acta 1790, 1117-1123. Doi: 10.1016/j.bbagen.2009.01.008. 
Imai, S., Guarente, L., 2010. Ten years of NAD-dependent SIR2 family deacetylases: implications for metabolic diseases. Trends Pharmacol Sci 31, 212-220. Doi: 10.1016/j.tips.2010.02.003.

Inoki, K., Zhu, T., Guan, K.L., 2003. TSC2 mediates cellular energy response to control cell growth and survival. Cell 115, 577-590. Doi.

Ishihara, M., Urushido, M., Hamada, K., Matsumoto, T., Shimamura, Y., Ogata, K., Inoue, K., Taniguchi, Y., Horino, T., Fujieda, M., Fujimoto, S., Terada, Y., 2013. Sestrin-2 and BNIP3 regulate autophagy and mitophagy in renal tubular cells in acute kidney injury. Am J Physiol Renal Physiol 305, F495-509. Doi: 10.1152/ajprenal.00642.2012.

Jager, S., Handschin, C., St-Pierre, J., Spiegelman, B.M., 2007. AMP-activated protein kinase (AMPK) action in skeletal muscle via direct phosphorylation of PGC-1alpha. Proc Natl Acad Sci U S A 104, 12017-12022. Doi: 10.1073/pnas.0705070104.

Jheng, H.F., Huang, S.H., Kuo, H.M., Hughes, M.W., Tsai, Y.S., 2015. Molecular insight and pharmacological approaches targeting mitochondrial dynamics in skeletal muscle during obesity. Ann N Y Acad Sci 1350, 82-94. Doi: 10.1111/nyas. 12863 .

Jheng, H.F., Tsai, P.J., Guo, S.M., Kuo, L.H., Chang, C.S., Su, I.J., Chang, C.R., Tsai, Y.S., 2012. Mitochondrial fission contributes to mitochondrial dysfunction and insulin resistance in skeletal muscle. Mol Cell Biol 32, 309-319. Doi: 10.1128/MCB.05603-11.

Jin, J., Gu, H., Anders, N.M., Ren, T., Jiang, M., Tao, M., Peng, Q., Rudek, M.A., Duan, W., 2016. Metformin Protects Cells from Mutant Huntingtin Toxicity 
Through Activation of AMPK and Modulation of Mitochondrial Dynamics. Neuromolecular Med. Doi: 10.1007/s12017-016-8412-z.

Jin, S.H., Yang, J.H., Shin, B.Y., Seo, K., Shin, S.M., Cho, I.J., Ki, S.H., 2013. Resveratrol inhibits LXRalpha-dependent hepatic lipogenesis through novel antioxidant Sestrin2 gene induction. Toxicol Appl Pharmacol 271, 95-105. Doi: 10.1016/j.taap.2013.04.023.

Joseph, S.B., Laffitte, B.A., Patel, P.H., Watson, M.A., Matsukuma, K.E., Walczak, R., Collins, J.L., Osborne, T.F., Tontonoz, P., 2002. Direct and indirect mechanisms for regulation of fatty acid synthase gene expression by liver $\mathrm{X}$ receptors. J Biol Chem 277, 11019-11025. Doi: 10.1074/jbc.M111041200.

Kammeyer, A., Luiten, R.M., 2015. Oxidation events and skin aging. Ageing Res Rev 21, 16-29. Doi: 10.1016/j.arr.2015.01.001.

Kang, T., Lu, W., Xu, W., Anderson, L., Bacanamwo, M., Thompson, W., Chen, Y.E., Liu, D., 2013. MicroRNA-27 (miR-27) targets prohibitin and impairs adipocyte differentiation and mitochondrial function in human adipose-derived stem cells. J Biol Chem 288, 34394-34402. Doi: 10.1074/jbc.M113.514372.

Karakelides, H., Irving, B.A., Short, K.R., O'Brien, P., Nair, K.S., 2010. Age, obesity, and sex effects on insulin sensitivity and skeletal muscle mitochondrial function. Diabetes 59, 89-97. Doi: 10.2337/db09-0591.

Kendrick, A.A., Choudhury, M., Rahman, S.M., McCurdy, C.E., Friederich, M., Van Hove, J.L., Watson, P.A., Birdsey, N., Bao, J., Gius, D., Sack, M.N., Jing, E., Kahn, C.R., Friedman, J.E., Jonscher, K.R., 2011. Fatty liver is associated with reduced SIRT3 activity and mitochondrial protein hyperacetylation. Biochem J 433, 505-514. Doi: 10.1042/BJ20100791. 
Kennedy, B.K., Berger, S.L., Brunet, A., Campisi, J., Cuervo, A.M., Epel, E.S., Franceschi, C., Lithgow, G.J., Morimoto, R.I., Pessin, J.E., Rando, T.A., Richardson, A., Schadt, E.E., Wyss-Coray, T., Sierra, F., 2014. Geroscience: linking aging to chronic disease. Cell 159, 709-713. Doi: 10.1016/j.cell.2014.10.039.

Kim, J., Kundu, M., Viollet, B., Guan, K.L., 2011. AMPK and mTOR regulate autophagy through direct phosphorylation of Ulk1. Nat Cell Biol 13, 132-141. Doi: $10.1038 / \mathrm{ncb} 2152$.

Kirkland, J.L., Tchkonia, T., Pirtskhalava, T., Han, J., Karagiannides, I., 2002. Adipogenesis and aging: does aging make fat go MAD? Exp Gerontol 37, 757767. Doi.

Koliaki, C., Roden, M., 2016. Alterations of Mitochondrial Function and Insulin Sensitivity in Human Obesity and Diabetes Mellitus. Annu Rev Nutr. Doi: 10.1146/annurev-nutr-071715-050656.

Kovalenko, E.I., Boyko, A.A., Semenkov, V.F., Lutsenko, G.V., Grechikhina, M.V., Kanevskiy, L.M., Azhikina, T.L., Telford, W.G., Sapozhnikov, A.M., 2014. ROS production, intracellular HSP70 levels and their relationship in human neutrophils: effects of age. Oncotarget 5, 11800-11812. Doi: 10.18632/oncotarget.2856.

Kuk, J.L., Saunders, T.J., Davidson, L.E., Ross, R., 2009. Age-related changes in total and regional fat distribution. Ageing Res Rev 8, 339-348. Doi: 10.1016/j.arr.2009.06.001.

Kume, S., Uzu, T., Horiike, K., Chin-Kanasaki, M., Isshiki, K., Araki, S., Sugimoto, T., Haneda, M., Kashiwagi, A., Koya, D., 2010. Calorie restriction enhances cell adaptation to hypoxia through Sirt1-dependent mitochondrial 
autophagy in mouse aged kidney. J Clin Invest 120, 1043-1055. Doi: 10.1172/JCI41376.

Lane, R.K., Hilsabeck, T., Rea, S.L., 2015. The role of mitochondrial dysfunction in age-related diseases. Biochim Biophys Acta 1847, 1387-1400. Doi: 10.1016/j.bbabio.2015.05.021.

Laplante, M., Sabatini, D.M., 2009. mTOR signaling at a glance. J Cell Sci 122, 3589-3594. Doi: 10.1242/jcs.051011.

Larsen, S., Hey-Mogensen, M., Rabol, R., Stride, N., Helge, J.W., Dela, F., 2012. The influence of age and aerobic fitness: effects on mitochondrial respiration in skeletal muscle. Acta Physiol (Oxf) 205, 423-432. Doi: 10.1111/j.1748-1716.2012.02408.x.

Larson-Meyer, D.E., Heilbronn, L.K., Redman, L.M., Newcomer, B.R., Frisard, M.I., Anton, S., Smith, S.R., Alfonso, A., Ravussin, E., 2006. Effect of calorie restriction with or without exercise on insulin sensitivity, beta-cell function, fat cell size, and ectopic lipid in overweight subjects. Diabetes Care 29, 1337-1344. Doi: $10.2337 / \mathrm{dc} 05-2565$.

Lee, C., Kim, K.H., Cohen, P., 2016. MOTS-c: A novel mitochondrial-derived peptide regulating muscle and fat metabolism. Free Radic Biol Med. Doi: 10.1016/j.freeradbiomed.2016.05.015.

Lee, C., Yen, K., Cohen, P., 2013a. Humanin: a harbinger of mitochondrialderived peptides? Trends Endocrinol Metab 24, 222-228. Doi: 10.1016/j.tem.2013.01.005.

Lee, C., Zeng, J., Drew, B.G., Sallam, T., Martin-Montalvo, A., Wan, J., Kim, S.J., Mehta, H., Hevener, A.L., de Cabo, R., Cohen, P., 2015. The mitochondrial-derived peptide MOTS-c promotes metabolic homeostasis and 
reduces obesity and insulin resistance. Cell Metab 21, 443-454. Doi: 10.1016/j.cmet.2015.02.009.

Lee, J.H., Budanov, A.V., Karin, M., 2013b. Sestrins orchestrate cellular metabolism to attenuate aging. Cell Metab 18, 792-801. Doi: 10.1016/j.cmet.2013.08.018.

Lee, J.H., Budanov, A.V., Park, E.J., Birse, R., Kim, T.E., Perkins, G.A., Ocorr, K., Ellisman, M.H., Bodmer, R., Bier, E., Karin, M., 2010. Sestrin as a feedback inhibitor of TOR that prevents age-related pathologies. Science 327, 12231228. Doi: $10.1126 /$ science. 1182228.

Lee, J.H., Budanov, A.V., Talukdar, S., Park, E.J., Park, H.L., Park, H.W., Bandyopadhyay, G., Li, N., Aghajan, M., Jang, I., Wolfe, A.M., Perkins, G.A., Ellisman, M.H., Bier, E., Scadeng, M., Foretz, M., Viollet, B., Olefsky, J., Karin, M., 2012. Maintenance of metabolic homeostasis by Sestrin2 and Sestrin3. Cell Metab 16, 311-321. Doi: 10.1016/j.cmet.2012.08.004.

Lee, S., Jeong, S.Y., Lim, W.C., Kim, S., Park, Y.Y., Sun, X., Youle, R.J., Cho, H., 2007. Mitochondrial fission and fusion mediators, hFis1 and OPA1, modulate cellular senescence. J Biol Chem 282, 22977-22983. Doi: 10.1074/jbc.M700679200.

Lee, T.H., Pastorino, L., Lu, K.P., 2011. Peptidyl-prolyl cis-trans isomerase Pin1 in ageing, cancer and Alzheimer disease. Expert Rev Mol Med 13, e21. Doi: 10.1017/S1462399411001906.

Li, G.H., Lin, X.L., Zhang, H., Li, S., He, X.L., Zhang, K., Peng, J., Tang, Y.L., Zeng, J.F., Zhao, Y., Ma, X.F., Lei, J.J., Wang, R., Wei, D.H., Jiang, Z.S., Wang, Z., 2015. Ox-Lp(a) transiently induces HUVEC autophagy via an ROS- 
dependent PAPR-1-LKB1-AMPK-mTOR pathway. Atherosclerosis 243, 223235. Doi: 10.1016/j.atherosclerosis.2015.09.020.

Li, Y., Xu, S., Mihaylova, M.M., Zheng, B., Hou, X., Jiang, B., Park, O., Luo, Z., Lefai, E., Shyy, J.Y., Gao, B., Wierzbicki, M., Verbeuren, T.J., Shaw, R.J., Cohen, R.A., Zang, M., 2011. AMPK phosphorylates and inhibits SREBP activity to attenuate hepatic steatosis and atherosclerosis in diet-induced insulin-resistant mice. Cell Metab 13, 376-388. Doi: 10.1016/j.cmet.2011.03.009.

Liesa, M., Borda-d'Agua, B., Medina-Gomez, G., Lelliott, C.J., Paz, J.C., Rojo, M., Palacin, M., Vidal-Puig, A., Zorzano, A., 2008. Mitochondrial fusion is increased by the nuclear coactivator PGC-1beta. PLoS One 3, e3613. Doi: 10.1371/journal.pone.0003613.

Liesa, M., Palacin, M., Zorzano, A., 2009. Mitochondrial dynamics in mammalian health and disease. Physiol Rev 89, 799-845. Doi: 10.1152/physrev.00030.2008.

Liu, X.M., Chan, H.C., Ding, G.L., Cai, J., Song, Y., Wang, T.T., Zhang, D., Chen, H., Yu, M.K., Wu, Y.T., Qu, F., Liu, Y., Lu, Y.C., Adashi, E.Y., Sheng, J.Z., Huang, H.F., 2015. FSH regulates fat accumulation and redistribution in aging through the Galphai/Ca(2+)/CREB pathway. Aging Cell 14, 409-420. Doi: 10.1111/acel.12331.

Liu, Y., Diaz, V., Fernandez, E., Strong, R., Ye, L., Baur, J.A., Lamming, D.W., Richardson, A., Salmon, A.B., 2014. Rapamycin-induced metabolic defects are reversible in both lean and obese mice. Aging (Albany NY) 6, 742-754. Doi: 10.18632/aging. 100688. 
Lokireddy, S., Wijesoma, I.W., Teng, S., Bonala, S., Gluckman, P.D., McFarlane, C., Sharma, M., Kambadur, R., 2012. The ubiquitin ligase Mul1 induces mitophagy in skeletal muscle in response to muscle-wasting stimuli. Cell Metab 16, 613-624. Doi: 10.1016/j.cmet.2012.10.005.

Lopez-Lluch, G., Irusta, P.M., Navas, P., de Cabo, R., 2008. Mitochondrial biogenesis and healthy aging. Exp Gerontol 43, 813-819. Doi: 10.1016/j.exger.2008.06.014.

Lopez-Lluch, G., Navas, P., 2015. Calorie restriction as an intervention in ageing. J Physiol. Doi: 10.1113/JP270543.

Lopez-Lluch, G., Navas, P., 2016. Calorie restriction as an intervention in ageing. J Physiol 594, 2043-2060. Doi: 10.1113/JP270543.

Lopez-Otin, C., Blasco, M.A., Partridge, L., Serrano, M., Kroemer, G., 2013. The hallmarks of aging. Cell 153, 1194-1217. Doi: 10.1016/j.cell.2013.05.039. Loson, O.C., Song, Z., Chen, H., Chan, D.C., 2013. Fis1, Mff, MiD49, and MiD51 mediate Drp1 recruitment in mitochondrial fission. Mol Biol Cell 24, 659667. Doi: 10.1091/mbc.E12-10-0721.

Lourenco, A.B., Munoz-Jimenez, C., Venegas-Caleron, M., Artal-Sanz, M., 2015. Analysis of the effect of the mitochondrial prohibitin complex, a contextdependent modulator of longevity, on the C. elegans metabolome. Biochim Biophys Acta 1847, 1457-1468. Doi: 10.1016/j.bbabio.2015.06.003.

Lowell, B.B., Shulman, G.I., 2005. Mitochondrial dysfunction and type 2 diabetes. Science 307, 384-387. Doi: 10.1126/science.1104343.

Lu, T., Finkel, T., 2008. Free radicals and senescence. Exp Cell Res 314, 19181922. Doi: 10.1016/j.yexcr.2008.01.011. 
Lupfer, C.R., Kanneganti, T.D., 2012. The role of inflammasome modulation in virulence. Virulence 3, 262-270. Doi: 10.4161/viru.20266.

Mahboobi, H., Golmirzaei, J., Gan, S.H., Jalalian, M., Kamal, M.A., 2014. Humanin: a possible linkage between Alzheimer's disease and type 2 diabetes. CNS Neurol Disord Drug Targets 13, 543-552. Doi.

Mai, S., Klinkenberg, M., Auburger, G., Bereiter-Hahn, J., Jendrach, M., 2010. Decreased expression of Drp1 and Fis1 mediates mitochondrial elongation in senescent cells and enhances resistance to oxidative stress through PINK1. J Cell Sci 123, 917-926. Doi: 10.1242/jcs.059246.

Martin-Montalvo, A., de Cabo, R., 2013. Mitochondrial metabolic reprogramming induced by calorie restriction. Antioxid Redox Signal 19, 310320. Doi: $10.1089 /$ ars.2012.4866.

Matsumoto, A.M., 2002. Andropause: clinical implications of the decline in serum testosterone levels with aging in men. J Gerontol A Biol Sci Med Sci 57, M76-99. Doi.

Mattagajasingh, I., Kim, C.S., Naqvi, A., Yamamori, T., Hoffman, T.A., Jung, S.B., DeRicco, J., Kasuno, K., Irani, K., 2007. SIRT1 promotes endotheliumdependent vascular relaxation by activating endothelial nitric oxide synthase. Proc Natl Acad Sci U S A 104, 14855-14860. Doi: 10.1073/pnas.0704329104.

McDonagh, B., 2016. Editorial: Redox Regulation in Skeletal Muscle Aging and Exercise. Front Physiol 7, 5. Doi: 10.3389/fphys.2016.00005.

McRae, S., Iqbal, J., Sarkar-Dutta, M., Lane, S., Nagaraj, A., Ali, N., Waris, G., 2015. Hepatitis C virus-induced NLRP3-Inflammasome Activates the Sterol Regulatory Element Binding Protein (SREBP) and Regulates Lipid Metabolism. J Biol Chem. Doi: 10.1074/jbc.M115.694059. 
Mercken, E.M., Carboneau, B.A., Krzysik-Walker, S.M., de Cabo, R., 2012. Of mice and men: the benefits of caloric restriction, exercise, and mimetics. Ageing Res Rev 11, 390-398. Doi: 10.1016/j.arr.2011.11.005.

Merkwirth, C., Martinelli, P., Korwitz, A., Morbin, M., Bronneke, H.S., Jordan, S.D., Rugarli, E.I., Langer, T., 2012. Loss of prohibitin membrane scaffolds impairs mitochondrial architecture and leads to tau hyperphosphorylation and neurodegeneration. PLoS Genet 8, e1003021. Doi: 10.1371/journal.pgen.1003021.

Merry, B.J., 2002. Molecular mechanisms linking calorie restriction and longevity. Int J Biochem Cell Biol 34, 1340-1354. Doi.

Mootha, V.K., Lindgren, C.M., Eriksson, K.F., Subramanian, A., Sihag, S., Lehar, J., Puigserver, P., Carlsson, E., Ridderstrale, M., Laurila, E., Houstis, N., Daly, M.J., Patterson, N., Mesirov, J.P., Golub, T.R., Tamayo, P., Spiegelman, B., Lander, E.S., Hirschhorn, J.N., Altshuler, D., Groop, L.C., 2003. PGC1alpha-responsive genes involved in oxidative phosphorylation are coordinately downregulated in human diabetes. Nat Genet 34, 267-273. Doi: 10.1038/ng1180.

Mudali, S., Dobs, A.S., 2004. Effects of testosterone on body composition of the aging male. Mech Ageing Dev 125, 297-304. Doi: 10.1016/j.mad.2004.01.004. Nakatsu, Y., Iwashita, M., Sakoda, H., Ono, H., Nagata, K., Matsunaga, Y., Fukushima, T., Fujishiro, M., Kushiyama, A., Kamata, H., Takahashi, S., Katagiri, H., Honda, H., Kiyonari, H., Uchida, T., Asano, T., 2015. Prolyl isomerase Pin1 negatively regulates AMP-activated protein kinase (AMPK) by associating with the CBS domain in the gamma subunit. J Biol Chem 290, 24255-24266. Doi: 10.1074/jbc.M115.658559. 
Nakatsu, Y., Sakoda, H., Kushiyama, A., Zhang, J., Ono, H., Fujishiro, M., Kikuchi, T., Fukushima, T., Yoneda, M., Ohno, H., Horike, N., Kanna, M., Tsuchiya, Y., Kamata, H., Nishimura, F., Isobe, T., Ogihara, T., Katagiri, H., Oka, Y., Takahashi, S., Kurihara, H., Uchida, T., Asano, T., 2011. Peptidylprolyl cis/trans isomerase NIMA-interacting 1 associates with insulin receptor substrate-1 and enhances insulin actions and adipogenesis. J Biol Chem 286, 20812-20822. Doi: 10.1074/jbc.M110.206904.

Nasrallah, C.M., Horvath, T.L., 2014. Mitochondrial dynamics in the central regulation of metabolism. Nat Rev Endocrinol 10, 650-658. Doi: 10.1038/nrendo.2014.160.

Nemoto, S., Fergusson, M.M., Finkel, T., 2005. SIRT1 functionally interacts with the metabolic regulator and transcriptional coactivator PGC-1\{alpha\}. J Biol Chem 280, 16456-16460. Doi: 10.1074/jbc.M501485200.

Newsholme, P., de Bittencourt, P.I., Jr., 2014. The fat cell senescence hypothesis: a mechanism responsible for abrogating the resolution of inflammation in chronic disease. Curr Opin Clin Nutr Metab Care 17, 295-305. Doi: 10.1097/MCO.0000000000000077.

Nisoli, E., Tonello, C., Cardile, A., Cozzi, V., Bracale, R., Tedesco, L., Falcone, S., Valerio, A., Cantoni, O., Clementi, E., Moncada, S., Carruba, M.O., 2005. Calorie restriction promotes mitochondrial biogenesis by inducing the expression of eNOS. Science 310, 314-317. Doi: 10.1126/science.1117728.

Palacios, O.M., Carmona, J.J., Michan, S., Chen, K.Y., Manabe, Y., Ward, J.L., 3rd, Goodyear, L.J., Tong, Q., 2009. Diet and exercise signals regulate SIRT3 and activate AMPK and PGC-1alpha in skeletal muscle. Aging (Albany NY) 1, 771-783. Doi. 
Palmer, B.F., Clegg, D.J., 2015. The sexual dimorphism of obesity. Mol Cell Endocrinol 402, 113-119. Doi: 10.1016/j.mce.2014.11.029.

Palus, S., von Haehling, S., Springer, J., 2014. Muscle wasting: an overview of recent developments in basic research. J Cachexia Sarcopenia Muscle 5, 193198. Doi: 10.1007/s13539-014-0157-7.

Park, Y.Y., Lee, S., Karbowski, M., Neutzner, A., Youle, R.J., Cho, H., 2010. Loss of MARCH5 mitochondrial E3 ubiquitin ligase induces cellular senescence through dynamin-related protein 1 and mitofusin 1 . J Cell Sci 123, 619-626. Doi: $10.1242 / \mathrm{jcs} .061481$.

Patti, M.E., Corvera, S., 2010. The role of mitochondria in the pathogenesis of type 2 diabetes. Endocr Rev 31, 364-395. Doi: 10.1210/er.2009-0027.

Payne, B.A., Chinnery, P.F., 2015. Mitochondrial dysfunction in aging: Much progress but many unresolved questions. Biochim Biophys Acta 1847, 13471353. Doi: 10.1016/j.bbabio.2015.05.022.

Peng, J., Ren, K.D., Yang, J., Luo, X.J., 2016. Mitochondrial E3 ubiquitin ligase 1: A key enzyme in regulation of mitochondrial dynamics and functions. Mitochondrion 28, 49-53. Doi: 10.1016/j.mito.2016.03.007.

Pich, S., Bach, D., Briones, P., Liesa, M., Camps, M., Testar, X., Palacin, M., Zorzano, A., 2005. The Charcot-Marie-Tooth type 2A gene product, Mfn2, upregulates fuel oxidation through expression of OXPHOS system. Hum Mol Genet 14, 1405-1415. Doi: 10.1093/hmg/ddi149.

Psarra, A.M., Solakidi, S., Sekeris, C.E., 2006. The mitochondrion as a primary site of action of regulatory agents involved in neuroimmunomodulation. Ann N Y Acad Sci 1088, 12-22. Doi: 10.1196/annals.1366.019. 
Putti, R., Sica, R., Migliaccio, V., Lionetti, L., 2015. Diet impact on mitochondrial bioenergetics and dynamics. Front Physiol 6, 109. Doi: 10.3389/fphys.2015.00109.

Qiu, X., Brown, K., Hirschey, M.D., Verdin, E., Chen, D., 2010. Calorie restriction reduces oxidative stress by SIRT3-mediated SOD2 activation. Cell Metab 12, 662-667. Doi: 10.1016/j.cmet.2010.11.015.

Quiros, P.M., Mottis, A., Auwerx, J., 2016. Mitonuclear communication in homeostasis and stress. Nat Rev Mol Cell Biol 17, 213-226. Doi: 10.1038/nrm.2016.23.

Rattan, S.I., 2008. Hormesis in aging. Ageing Res Rev 7, 63-78. Doi: 10.1016/j.arr.2007.03.002.

Repa, J.J., Liang, G., Ou, J., Bashmakov, Y., Lobaccaro, J.M., Shimomura, I., Shan, B., Brown, M.S., Goldstein, J.L., Mangelsdorf, D.J., 2000. Regulation of mouse sterol regulatory element-binding protein-1c gene (SREBP-1c) by oxysterol receptors, LXRalpha and LXRbeta. Genes Dev 14, 2819-2830. Doi.

Rhee, S.G., Bae, S.H., 2015. The antioxidant function of sestrins is mediated by promotion of autophagic degradation of Keap1 and Nrf2 activation and by inhibition of mTORC1. Free Radic Biol Med 88, 205-211. Doi: 10.1016/j.freeradbiomed.2015.06.007.

Riera, C.E., Merkwirth, C., De Magalhaes Filho, C.D., Dillin, A., 2016. Signaling Networks Determining Life Span. Annu Rev Biochem 85, 35-64. Doi: 10.1146/annurev-biochem-060815-014451.

Ristow, M., 2014. Unraveling the truth about antioxidants: mitohormesis explains ROS-induced health benefits. Nat Med 20, 709-711. Doi: 10.1038/nm.3624. 
Rodriguez-Bies, E., Navas, P., Lopez-Lluch, G., 2015. Age-dependent effect of every-other-day feeding and aerobic exercise in ubiquinone levels and related antioxidant activities in mice muscle. J Gerontol A Biol Sci Med Sci 70, 33-43. Doi: $10.1093 /$ gerona/glu002.

Rodriguez-Bies, E., Tung, B.T., Navas, P., Lopez-Lluch, G., 2016. Resveratrol primes the effects of physical activity in old mice. Br J Nutr 116, 979-988. Doi: 10.1017/S0007114516002920.

Ryan, B.J., Hoek, S., Fon, E.A., Wade-Martins, R., 2015. Mitochondrial dysfunction and mitophagy in Parkinson's: from familial to sporadic disease. Trends Biochem Sci 40, 200-210. Doi: 10.1016/j.tibs.2015.02.003.

Samant, S.A., Zhang, H.J., Hong, Z., Pillai, V.B., Sundaresan, N.R., Wolfgeher, D., Archer, S.L., Chan, D.C., Gupta, M.P., 2014. SIRT3 deacetylates and activates OPA1 to regulate mitochondrial dynamics during stress. Mol Cell Biol 34, 807-819. Doi: 10.1128/MCB.01483-13.

Sanchis-Gomar, F., 2013. Sestrins: novel antioxidant and AMPK-modulating functions regulated by exercise? J Cell Physiol 228, 1647-1650. Doi: 10.1002/jcp.24338.

Santa-Cruz Calvo, S., Navas, P., López-Lluch, G., 2012. Sirtuin-dependent meabolic control and its role in the aging process, In: Clark, K., B. (Ed.), Bioenergetics. InTech, Rijeka, Croatia, pp. 95-120.

Santanasto, A.J., Goodpaster, B.H., Kritchevsky, S.B., Miljkovic, I., Satterfield, S., Schwartz, A.V., Cummings, S.R., Boudreau, R.M., Harris, T.B., Newman, A.B., 2016. Body Composition Remodeling and Mortality: The Health Aging and Body Composition Study. J Gerontol A Biol Sci Med Sci. Doi: 10.1093/gerona/glw163. 
Santel, A., Frank, S., 2008. Shaping mitochondria: The complex posttranslational regulation of the mitochondrial fission protein DRP1. IUBMB Life 60, 448-455. Doi: 10.1002/iub.71.

Sastre-Serra, J., Nadal-Serrano, M., Pons, D.G., Roca, P., Oliver, J., 2012. Mitochondrial dynamics is affected by 17 beta-estradiol in the MCF-7 breast cancer cell line. Effects on fusion and fission related genes. Int $\mathrm{J}$ Biochem Cell Biol 44, 1901-1905. Doi: 10.1016/j.biocel.2012.07.012.

Sastre-Serra, J., Nadal-Serrano, M., Pons, D.G., Roca, P., Oliver, J., 2013. The over-expression of ERbeta modifies estradiol effects on mitochondrial dynamics in breast cancer cell line. Int J Biochem Cell Biol 45, 1509-1515. Doi: 10.1016/j.biocel.2013.04.007.

Sataranatarajan, K., Ikeno, Y., Bokov, A., Feliers, D., Yalamanchili, H., Lee, H.J., Mariappan, M.M., Tabatabai-Mir, H., Diaz, V., Prasad, S., Javors, M.A., Ghosh Choudhury, G., Hubbard, G.B., Barnes, J.L., Richardson, A., Kasinath, B.S., 2016. Rapamycin Increases Mortality in $\mathrm{db} / \mathrm{db}$ Mice, a Mouse Model of Type 2 Diabetes. J Gerontol A Biol Sci Med Sci 71, 850-857. Doi: 10.1093/gerona/glv170.

Scarpace, P.J., Matheny, M., Strehler, K.Y., Toklu, H.Z., Kirichenko, N., Carter, C.S., Morgan, D., Tumer, N., 2016. Rapamycin Normalizes Serum Leptin by Alleviating Obesity and Reducing Leptin Synthesis in Aged Rats. J Gerontol A Biol Sci Med Sci 71, 891-899. Doi: 10.1093/gerona/glu230.

Scialo, F., Sriram, A., Fernandez-Ayala, D., Gubina, N., Lohmus, M., Nelson, G., Logan, A., Cooper, H.M., Navas, P., Enriquez, J.A., Murphy, M.P., Sanz, A., 2016. Mitochondrial ROS Produced via Reverse Electron Transport Extend Animal Lifespan. Cell Metab 23, 725-734. Doi: 10.1016/j.cmet.2016.03.009. 
Scheller, K., Seibel, P., Sekeris, C.E., 2003. Glucocorticoid and thyroid hormone receptors in mitochondria of animal cells. Int Rev Cytol 222, 1-61. Doi. Schneeberger, M., Dietrich, M.O., Sebastian, D., Imbernon, M., Castano, C., Garcia, A., Esteban, Y., Gonzalez-Franquesa, A., Rodriguez, I.C., Bortolozzi, A., Garcia-Roves, P.M., Gomis, R., Nogueiras, R., Horvath, T.L., Zorzano, A., Claret, M., 2013. Mitofusin 2 in POMC neurons connects ER stress with leptin resistance and energy imbalance. Cell 155, 172-187. Doi: 10.1016/j.cell.2013.09.003.

Sekeris, C.E., 1990. The mitochondrial genome: a possible primary site of action of steroid hormones. In Vivo 4, 317-320. Doi.

Senesi, P., Montesano, A., Luzi, L., Codella, R., Benedini, S., Terruzzi, I., 2016. Metformin Treatment Prevents Sedentariness Related Damages in Mice. J Diabetes Res 2016, 8274689. Doi: 10.1155/2016/8274689.

Shen, Q., Yamano, K., Head, B.P., Kawajiri, S., Cheung, J.T., Wang, C., Cho, J.H., Hattori, N., Youle, R.J., van der Bliek, A.M., 2014. Mutations in Fis1 disrupt orderly disposal of defective mitochondria. Mol Biol Cell 25, 145-159. Doi: 10.1091/mbc.E13-09-0525.

Short, K.R., Bigelow, M.L., Kahl, J., Singh, R., Coenen-Schimke, J., Raghavakaimal, S., Nair, K.S., 2005. Decline in skeletal muscle mitochondrial function with aging in humans. Proc Natl Acad Sci U S A 102, 5618-5623. Doi: 10.1073/pnas.0501559102.

Simoneau, J.A., Veerkamp, J.H., Turcotte, L.P., Kelley, D.E., 1999. Markers of capacity to utilize fatty acids in human skeletal muscle: relation to insulin resistance and obesity and effects of weight loss. FASEB J 13, 2051-2060. Doi. 
Smirnova, E., Griparic, L., Shurland, D.L., van der Bliek, A.M., 2001. Dynaminrelated protein Drp1 is required for mitochondrial division in mammalian cells. Mol Biol Cell 12, 2245-2256. Doi.

Soriano, F.X., Liesa, M., Bach, D., Chan, D.C., Palacin, M., Zorzano, A., 2006. Evidence for a mitochondrial regulatory pathway defined by peroxisome proliferator-activated receptor-gamma coactivator-1 alpha, estrogen-related receptor-alpha, and mitofusin 2. Diabetes 55, 1783-1791. Doi: 10.2337/db050509.

Sreekumar, P.G., Ishikawa, K., Spee, C., Mehta, H.H., Wan, J., Yen, K., Cohen, P., Kannan, R., Hinton, D.R., 2016. The Mitochondrial-Derived Peptide Humanin Protects RPE Cells From Oxidative Stress, Senescence, and Mitochondrial Dysfunction. Invest Ophthalmol Vis Sci 57, 1238-1253. Doi: 10.1167/iovs.15-17053.

Steinberg, G.R., O'Neill, H.M., Dzamko, N.L., Galic, S., Naim, T., Koopman, R., Jorgensen, S.B., Honeyman, J., Hewitt, K., Chen, Z.P., Schertzer, J.D., Scott, J.W., Koentgen, F., Lynch, G.S., Watt, M.J., van Denderen, B.J., Campbell, D.J., Kemp, B.E., 2010. Whole body deletion of AMP-activated protein kinase \{beta\}2 reduces muscle AMPK activity and exercise capacity. J Biol Chem 285, 37198-37209. Doi: 10.1074/jbc.M110.102434.

Stienstra, R., van Diepen, J.A., Tack, C.J., Zaki, M.H., van de Veerdonk, F.L., Perera, D., Neale, G.A., Hooiveld, G.J., Hijmans, A., Vroegrijk, I., van den Berg, S., Romijn, J., Rensen, P.C., Joosten, L.A., Netea, M.G., Kanneganti, T.D., 2011. Inflammasome is a central player in the induction of obesity and insulin resistance. Proc Natl Acad Sci U S A 108, 15324-15329. Doi: 10.1073/pnas. 1100255108. 
Storlien, L., Oakes, N.D., Kelley, D.E., 2004. Metabolic flexibility. Proc Nutr Soc 63, 363-368. Doi: 10.1079/PNS2004349.

Supale, S., Thorel, F., Merkwirth, C., Gjinovci, A., Herrera, P.L., Scorrano, L., Meda, P., Langer, T., Maechler, P., 2013. Loss of prohibitin induces mitochondrial damages altering beta-cell function and survival and is responsible for gradual diabetes development. Diabetes 62, 3488-3499. Doi: $10.2337 / \mathrm{db} 13-0152$.

Tao, R., Xiong, X., Liangpunsakul, S., Dong, X.C., 2015. Sestrin 3 protein enhances hepatic insulin sensitivity by direct activation of the mTORC2-Akt signaling. Diabetes 64, 1211-1223. Doi: 10.2337/db14-0539.

Theiss, A.L., Vijay-Kumar, M., Obertone, T.S., Jones, D.P., Hansen, J.M., Gewirtz, A.T., Merlin, D., Sitaraman, S.V., 2009. Prohibitin is a novel regulator of antioxidant response that attenuates colonic inflammation in mice. Gastroenterology 137, 199-208, 208 e191-196. Doi: 10.1053/j.gastro.2009.03.033.

Toledo, F.G., Goodpaster, B.H., 2013. The role of weight loss and exercise in correcting skeletal muscle mitochondrial abnormalities in obesity, diabetes and aging. Mol Cell Endocrinol 379, 30-34. Doi: 10.1016/j.mce.2013.06.018.

Tung, B.T., Rodriguez-Bies, E., Ballesteros-Simarro, M., Motilva, V., Navas, P., Lopez-Lluch, G., 2014. Modulation of endogenous antioxidant activity by resveratrol and exercise in mouse liver is age dependent. J Gerontol A Biol Sci Med Sci 69, 398-409. Doi: 10.1093/gerona/glt102.

Tung, B.T., Rodriguez-Bies, E., Talero, E., Gamero-Estevez, E., Motilva, V., Navas, P., Lopez-Lluch, G., 2015a. Anti-inflammatory effect of resveratrol in old mice liver. Exp Gerontol 64, 1-7. Doi: 10.1016/j.exger.2015.02.004. 
Tung, B.T., Rodriguez-Bies, E., Thanh, H.N., Le-Thi-Thu, H., Navas, P., Sanchez, V.M., Lopez-Lluch, G., 2015b. Organ and tissue-dependent effect of resveratrol and exercise on antioxidant defenses of old mice. Aging Clin Exp Res Doi: 10.1007/s40520-015-0366-8. Doi: 10.1007/s40520-015-0366-8.

Turner, N., Bruce, C.R., Beale, S.M., Hoehn, K.L., So, T., Rolph, M.S., Cooney, G.J., 2007. Excess lipid availability increases mitochondrial fatty acid oxidative capacity in muscle: evidence against a role for reduced fatty acid oxidation in lipid-induced insulin resistance in rodents. Diabetes 56, 2085-2092. Doi: 10.2337/db07-0093.

Twig, G., Elorza, A., Molina, A.J., Mohamed, H., Wikstrom, J.D., Walzer, G., Stiles, L., Haigh, S.E., Katz, S., Las, G., Alroy, J., Wu, M., Py, B.F., Yuan, J., Deeney, J.T., Corkey, B.E., Shirihai, O.S., 2008. Fission and selective fusion govern mitochondrial segregation and elimination by autophagy. EMBO J 27, 433-446. Doi: 10.1038/sj.emboj.7601963.

Vasconsuelo, A., Milanesi, L., Boland, R., 2013. Actions of 17 beta-estradiol and testosterone in the mitochondria and their implications in aging. Ageing Res Rev 12, 907-917. Doi: 10.1016/j.arr.2013.09.001.

Wai, T., Langer, T., 2016. Mitochondrial Dynamics and Metabolic Regulation. Trends Endocrinol Metab 27, 105-117. Doi: 10.1016/j.tem.2015.12.001.

Wallace, D.C., 2012. Mitochondria and cancer. Nat Rev Cancer 12, 685-698. Doi: $10.1038 / n r c 3365$.

Wang, C.H., Wang, C.C., Huang, H.C., Wei, Y.H., 2013. Mitochondrial dysfunction leads to impairment of insulin sensitivity and adiponectin secretion in adipocytes. FEBS J 280, 1039-1050. Doi: 10.1111/febs.12096. 
Weber, T.A., Reichert, A.S., 2010. Impaired quality control of mitochondria: aging from a new perspective. Exp Gerontol 45, 503-511. Doi: 10.1016/j.exger.2010.03.018.

Wullschleger, S., Loewith, R., Hall, M.N., 2006. TOR signaling in growth and metabolism. Cell 124, 471-484. Doi: 10.1016/j.cell.2006.01.016.

Yang, S.B., Tien, A.C., Boddupalli, G., Xu, A.W., Jan, Y.N., Jan, L.Y., 2012. Rapamycin ameliorates age-dependent obesity associated with increased mTOR signaling in hypothalamic POMC neurons. Neuron 75, 425-436. Doi: 10.1016/j.neuron.2012.03.043.

Yang, Y.L., Loh, K.S., Liou, B.Y., Chu, I.H., Kuo, C.J., Chen, H.D., Chen, C.S., 2013. SESN-1 is a positive regulator of lifespan in Caenorhabditis elegans. Exp Gerontol 48, 371-379. Doi: 10.1016/j.exger.2012.12.011.

Yoon, Y., Krueger, E.W., Oswald, B.J., McNiven, M.A., 2003. The mitochondrial protein hFis1 regulates mitochondrial fission in mammalian cells through an interaction with the dynamin-like protein DLP1. Mol Cell Biol 23, 5409-5420. Doi.

Yoon, Y.S., Yoon, D.S., Lim, I.K., Yoon, S.H., Chung, H.Y., Rojo, M., Malka, F., Jou, M.J., Martinou, J.C., Yoon, G., 2006. Formation of elongated giant mitochondria in DFO-induced cellular senescence: involvement of enhanced fusion process through modulation of Fis1. J Cell Physiol 209, 468-480. Doi: 10.1002/jcp.20753.

Yu, J., Nagasu, H., Murakami, T., Hoang, H., Broderick, L., Hoffman, H.M., Horng, T., 2014. Inflammasome activation leads to Caspase-1-dependent mitochondrial damage and block of mitophagy. Proc Natl Acad Sci U S A 111, 15514-15519. Doi: 10.1073/pnas.1414859111. 
Zamboni, M., Mazzali, G., Zoico, E., Harris, T.B., Meigs, J.B., Di Francesco, V., Fantin, F., Bissoli, L., Bosello, O., 2005. Health consequences of obesity in the elderly: a review of four unresolved questions. Int J Obes (Lond) 29, 1011-1029. Doi: $10.1038 /$ sj.ijo.0803005.

Ziegler, D.V., Wiley, C.D., Velarde, M.C., 2015. Mitochondrial effectors of cellular senescence: beyond the free radical theory of aging. Aging Cell 14, 1-7. Doi: 10.1111/acel.12287.

Zorzano, A., Claret, M., 2015. Implications of mitochondrial dynamics on neurodegeneration and on hypothalamic dysfunction. Front Aging Neurosci 7, 101. Doi: $10.3389 /$ fnagi.2015.00101.

Zorzano, A., Liesa, M., Palacin, M., 2009. Role of mitochondrial dynamics proteins in the pathophysiology of obesity and type 2 diabetes. Int $\mathrm{J}$ Biochem Cell Biol 41, 1846-1854. Doi: 10.1016/j.biocel.2009.02.004. 
CR EXERCISE

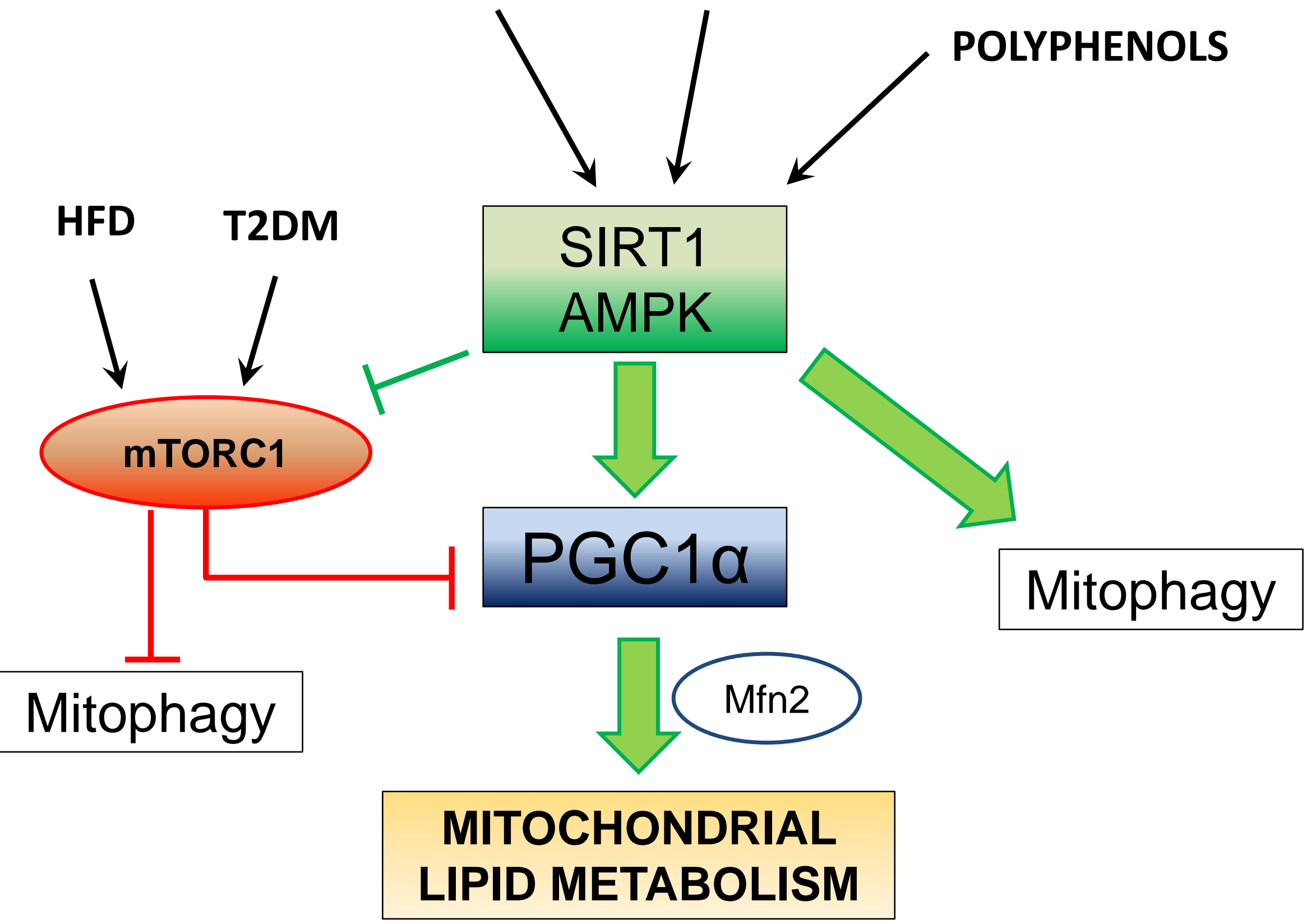




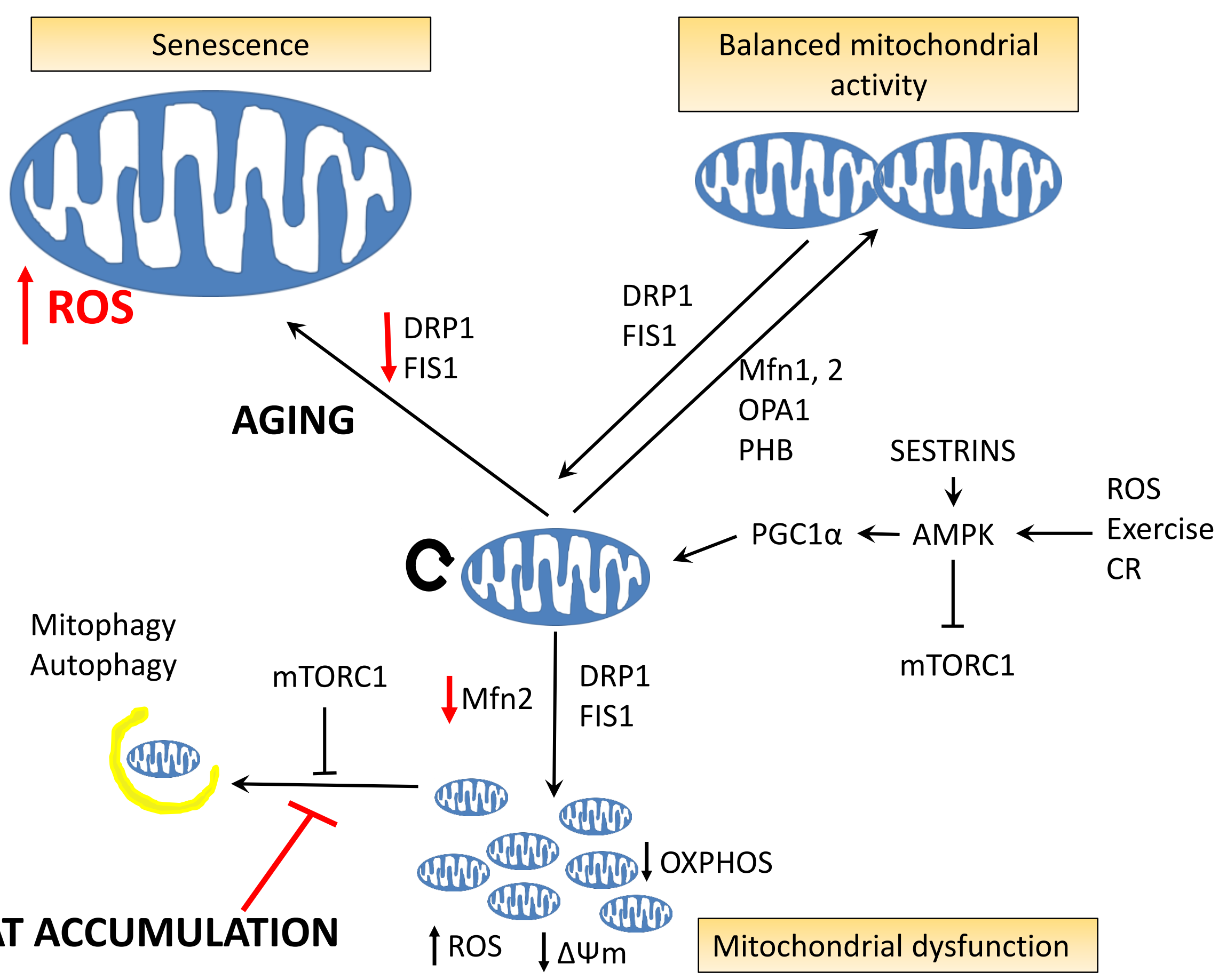




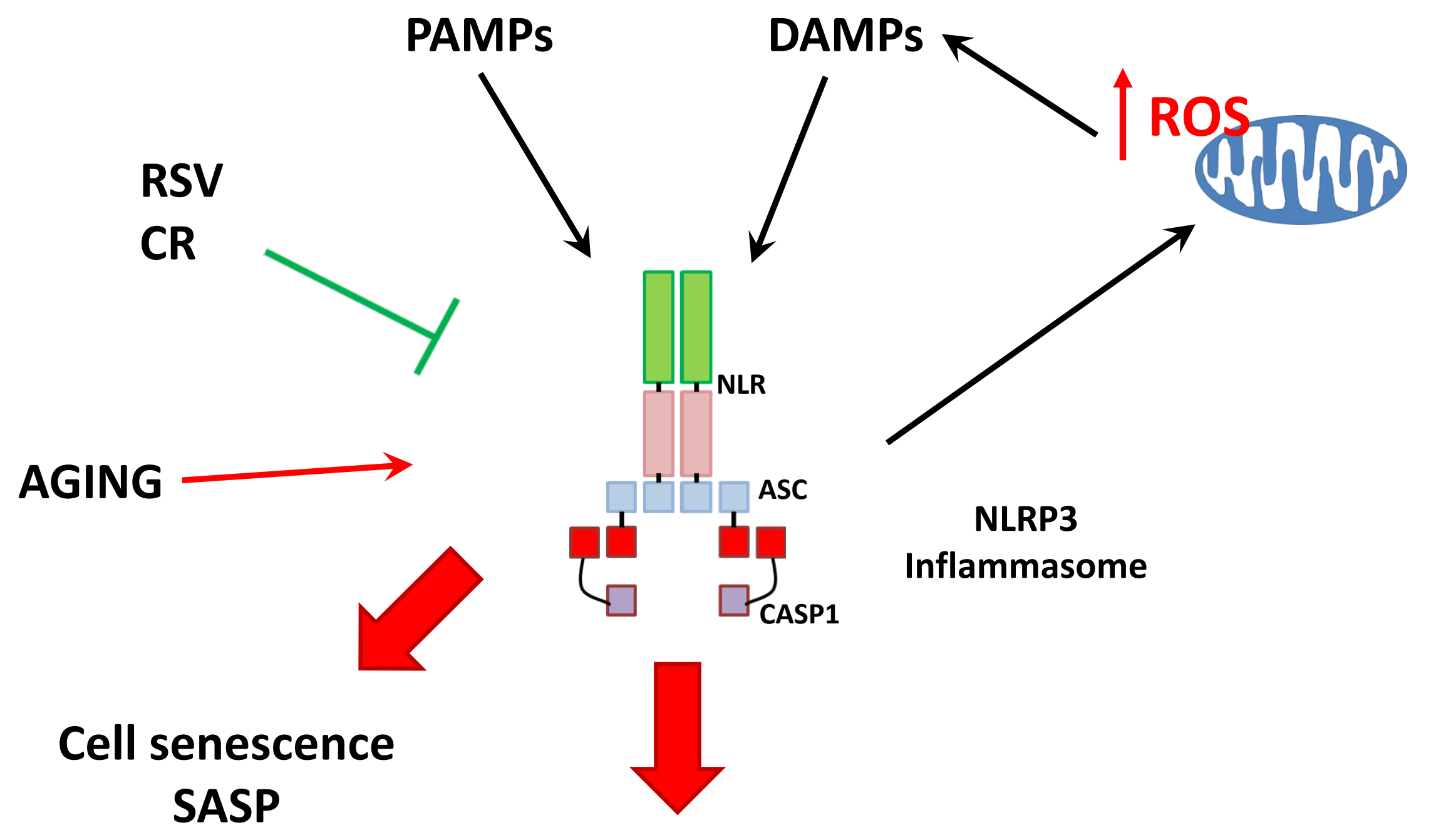

Hepatosteatosis Obesity

Insulin resistance 


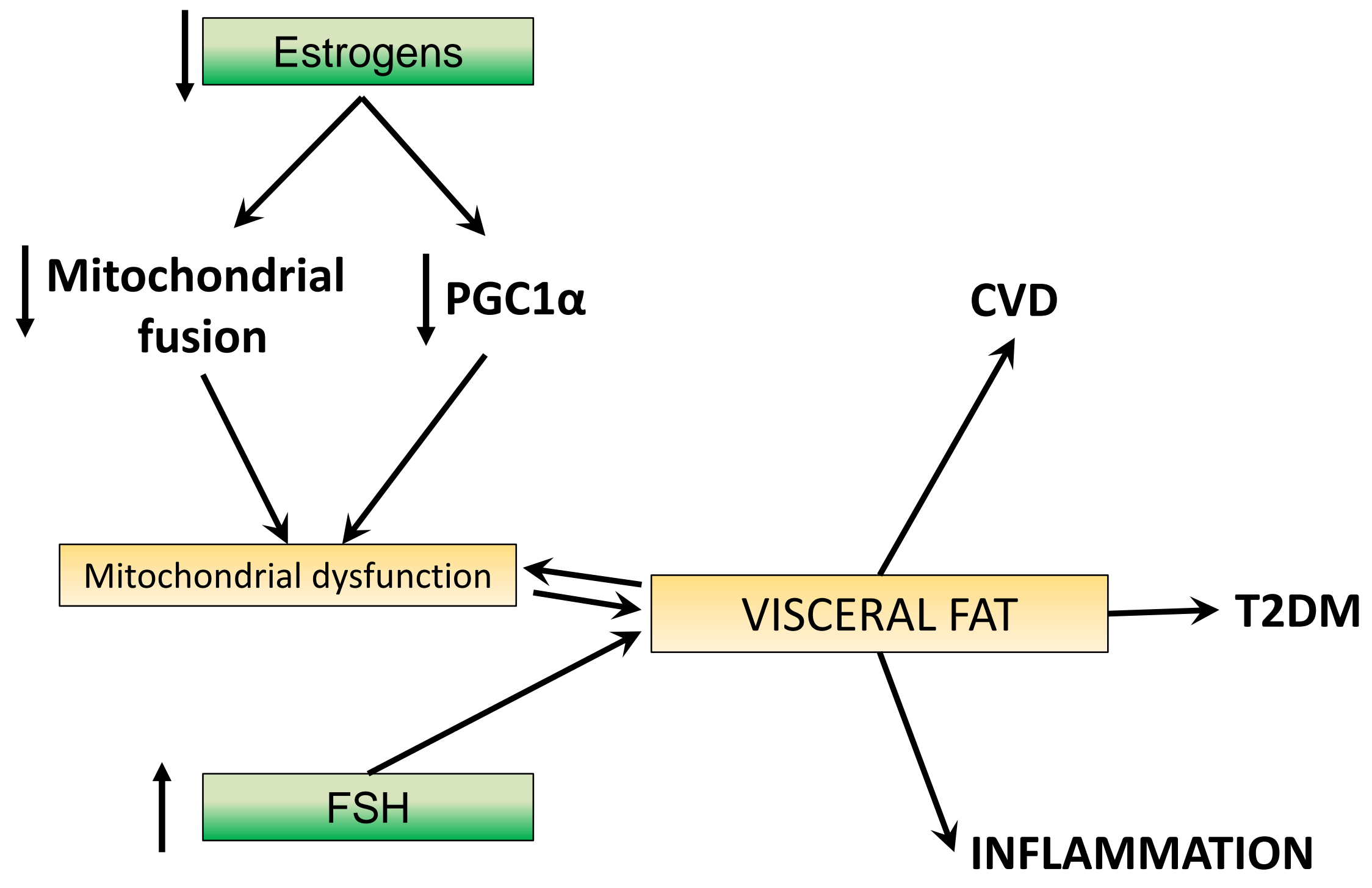




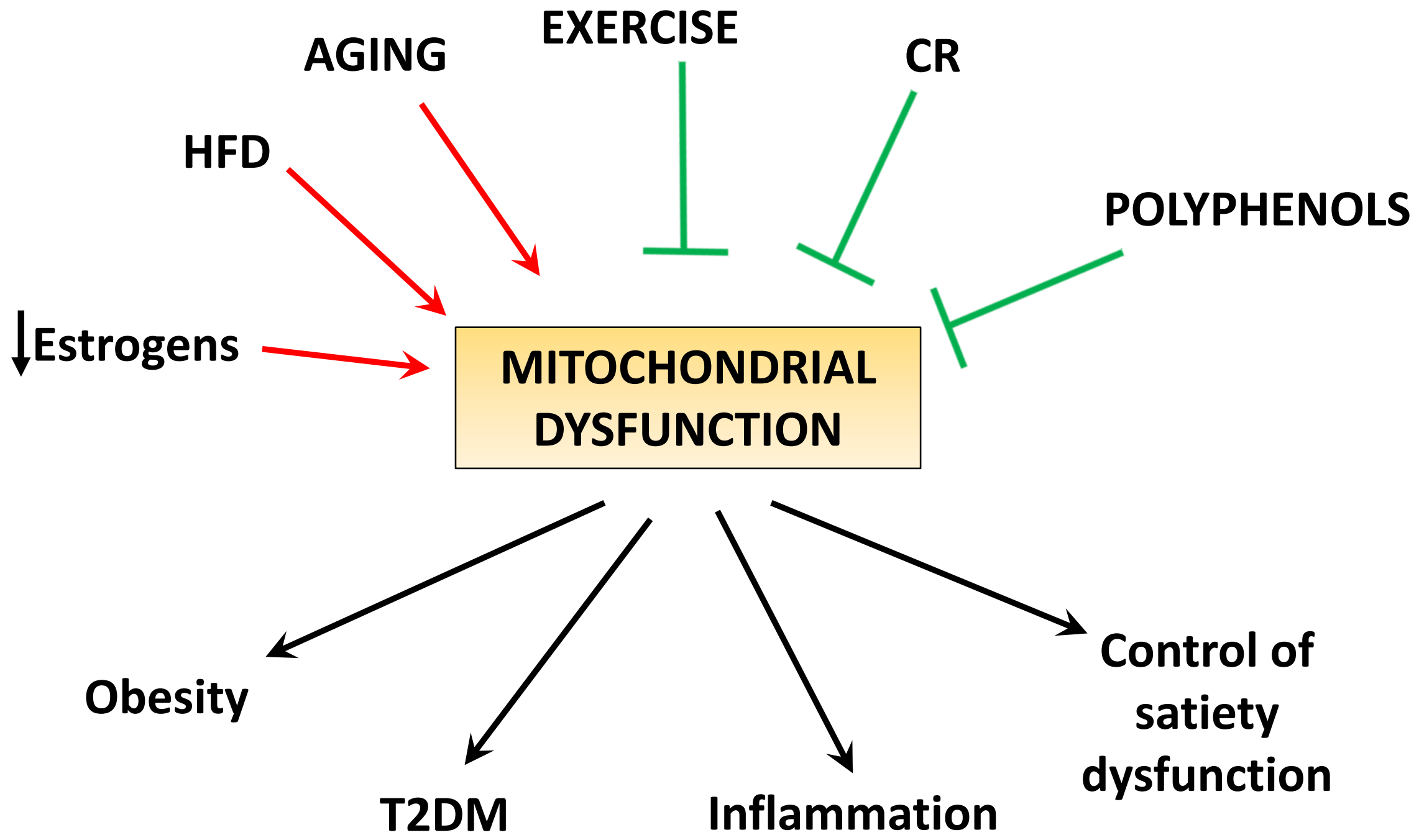


Highlights paper:

Mitochondrial dysfunction is associated with age.-related fat accumulation.

Mitochondrial dynamics are key components in fat and aging.

Mitochondrial damage induces inflammasome activity in adipocytes-derives proinflammatory cytokines.

Estrogens regulate mitochondrial activity and affect age-dependent fat depots. 
For: Mech Ageing Dev

\title{
Mitochondrial activity and dynamics in fatchanges regarding metabolism during-in ageing and obesity
}

\author{
Guillermo López-Lluch
}

Universidad Pablo de Olavide, Centro Andaluz de Biología del Desarrollo,

CABD-CSIC, CIBERER, Instituto de Salud Carlos III, Carretera de Utrera km. 1, 41013 Sevilla, Spain.

${ }^{*}$ Address correspondence:

Centro Andaluz de Biología del Desarrollo (CABD), Universidad Pablo de Olavide, Carretera de Utrera Km. 1, 41013 Sevilla, Spain. Email: glopllu@upo.es 


\section{Abstract}

Mitochondria play an essential role in ageing and longevity. During ageing, a general deregulation of metabolism occurs, affecting molecular, cellular and physiological functions-activities in the organism. Dysfunction of mitochondria has been associated with ageing and age-related diseases indicating their importance in the maintenance of cell homeostasis. Three mainmajor nutritional sensors, mTOR, AMPK and Sirtuins are involved in the control of mitochondrial physiology. These nutritional sensors control mitochondrial biogenesis, dynamics; by regulating fusion and fission processes, and turnover through mito- and autophagy. Apart of the known factors involved in fusion, OPA1 and mitofusins, and fission, DRP1 and FIS1, emerging factors such as prohibitins and sestrins can play important functions in mitochondrial dynamics regulation. Mitochondria are also affected by sexual hormones that suffer drastic changes during ageing. The recent literature demonstrates the complex interaction between nutritional sensors and mitochondrial homeostasis in the physiology of adipose tissue and in the accumulation of fat in other organs such as muscle and liver. In this article, the importance role of mitochondrial homeostasis in ageing and during age-dependent fat accumulation is revised. This review highlights the importance of mitochondria in the accumulation of fat during ageing and related diseases such as obesity, metabolic syndrome or type 2 diabetes mellitus.

Keywords: mitochondria; ageing; fat; gender; inflammation 


\section{Introduction}

Ageing is a natural and progressive process occurring in all living organisms and characterized by the deterioration of structure and functional capacities. Ageing is multifactorial involving different molecular and cellular processes such as genomic instability, telomere attrition, epigenetic alterations, loss of proteostasis, deregulated nutrient sensing, mitochondrial dysfunction, cellular senescence, stem cell exhaustion, and altered intracellular communication (Kennedy et al., 2014; Lopez-Otin et al., 2013). Ageing is also characterized by the appearance of different chronic diseases such as sarcopenia, type 2 diabetes mellitus (T2DM), cardiovascular disease, hypercholesterolemia, hypertension or neurodegenerative diseases among others (Kennedy et al., 2014).

A tendency towards higher visceral adiposity associated with a greater morbidity due to the increase of risk for cardiovascular disease (CVD), hypertension and T2DM is-has been associated with the progression of ageing (Matsumoto, 2002). Obesity is associated with adipose tissue dysfunction characterized by adipocyte hypertrophy, proinflammatory processes, impaired insulin signalling and insulin resistance (Karakelides et al., 2010). The incapacity decrease in the capacity to respond to insulin induces ectopic fat deposition in other organs producing lipotoxicity in muscle, liver and also in pancreas. Thus, the prevalence of obesity in older people has been considered the main-major cause of metabolic syndrome (Karakelides et al., 2010) and changes in adipose tissue have been recently associated with the development of T2DM (Abranches et al., 2015). Losses of lean mass and shifts to central 
adiposity have been related to functional decline and considered as predictors of mortality (Kirkland et al., 2002).

Fat depots sizes in humans reach a peak by middle and early old age (Cartwright et al., 2007). However, in advanced old age, a decrease in fat tissue occurs although this decrease has been associated with the accumulation of fat in liver, muscle and bone marrow and other organs (Kirkland et al., 2002). In a recent study performed in 70-79 years old men and women, it was determined that loss of weight was accompanied by the reduction of visceral fat mass accompanied by a gain of intermuscular thigh fat area (Santanasto et al., 2016). This redistribution of fat can be related with the preservation of thight muscle since this study demonstrated a strong relationship between loss of thight muscle and mortality whereas no relationship was found with visceral mass (Santanasto et al., 2016).

The role of mitochondria in fat reduction in older old people has been also suggested. In adipose tissue, dysfunctional adipose-like cells appear. These cells are smaller than normal adipocytes and show a low response to insulin. Their dedifferentiation occurs by the decrease of adipogenic factors such as $\underline{\text { CCAAT/enhancer binding alpha (C/EBPalpha) and peroxisome proliferator }}$ activated receptor gamma (PPAR-v), a known mitochondrial regulator (Kirkland et al., 2002).

Mitochondria are key organelles in the metabolism in of eukaryotic cells (LopezLluch et al., 2008). Their activity is modulated by the availability of energy in cells. Key regulators of metabolism not only affect the activity of mitochondria but also their dynamics and turnover. One of these regulators is the mammalian 
Target of Rapamycin (mTOR) which is activated by high calorie intake or high levels of aminoacids (Laplante and Sabatini, 2009). Opposite regulators are AMP-activated kinase (AMPK) and sirtuins that are activated when energy uptake is limited (Hardie, 2011).

The term mitochondrial dysfunction is widely used in bioenergetics although there is not a precise definition. Any abnormality in the key physiological functions of mitochondria: generation of ATP, detoxification of ROS and ROSrelated damage, regulation of cytosolic calcium levels, synthesis of lipids, etc., can be considered as mitochondrial dysfunction (Brand and Nicholls, 2011). Mitochondrial dysfunction is associated with the functional decline found in tissues and organs during ageing (Riera et al., 2016)_and, for this reason, it has been associated with a number of age-related diseases such as neurological disorders, cardiovascularCVD, liver and kidney diseases, diabetes and cancer (Lane et al., 2015; Payne and Chinnery, 2015; Ryan et al., 2015; Wallace, 2012). Recently, a study carried out with 248 non-diabetic participants from the Baltimore Londigutinal Study of Aging has associated impaired mitochondrial capacity with greater insulin resistance (Fabbri et al., 2016).

Information about the role of mitochondria on white adipose tissue (WAT) is limited in animal models and humans. Since the regulation of mitochondrial activity in WAT can be associated with the accumulation of fat and a putative disruption of mitochondrial physiology, the study of mitochondrial homeostasis is important to develop strategies or therapies to avoid or delay the progression of fat accumulation-derived diseases. For this reason, in this review, the role of mitochondrial activity, dynamics and turnover on fat accumulation in WAT, 
muscle or liver and the molecular factors involved in these processes is reviewed in deep.

Molecular mechanisms involved in the regulation of mitochondrial physiology.

Mitochondrial physiology, dynamics and turnover respond to the relationship between the activities of key regulators of cell metabolism. In high calorie intake situations, mTOR, an ubiquitous serine/threonine kinase involved in cell growth and the control of metabolism, is activated. This regulator is a complex of different proteins and regulates different pathways involved in angiogenesis, tumour development, insulin resistance, adipogenesis and activation of immune cells (Laplante and Sabatini, 2009). It is present in two different complexes, mTOR complex I (mTORC1) and mTOR complex II (mTORC2). Both complexes promote the transcription of genes involved in carbohydrate metabolism and lipogenesis, enhance protein translation by activating ribosomal protein 6 kinase (S6K) and, at the same time, downregulate catabolic processes through inhibiting the transcriptional activity of the forkhead box transcription factors family (FoxO) and autophagy (Duvel et al., 2010; Wullschleger et al., 2006).

The signalling pathway triggered by mTOR competes against other regulators of the cell metabolism such as AMPK and sirtuins, NAD+-dependent deacetylases that induce-inducing more efficient energy consumption in situations of low intake, starvation or calorie restriction (CR)-situations, such as AMPK and sirtuins, $\mathrm{NAD}^{ \pm}$-dependent deacetylases-(Fig. 1). AMPK is one of the 
mainmajor sensitive energy sensors in cells and organisms. This kinase is activated in response to the increase of the AMPIATP ratio, for example, after deprivation of glucose, whereas its activity is reduced when cells are full of energy and the AMP/ATP ratio decreases (Hardie, 2011).

Another of the most important sensors of energy in cells and regulators of mitochondrial activity are sirtuins. This family of $\mathrm{NAD}^{+}$-dependent deacetylases is found in all the organisms, from prokaryotes to humans, and regulates the activity of many enzymes by removing acetyl residues, except in the case of mammalian SIRT4 and SIRT5 that show ADP-ribosyl-transferase activity (Santa-Cruz Calvo et al., 2012). Sirtuins also act as metabolic sensors by detecting fluctuations in the $\mathrm{NAD}^{+} / \mathrm{NADH}$ ratio in cells. When the amount of nutrients decrease, especially in the case of glucose, levels of NAD+ increase and sirtuins are then activated. The number of proteins regulated by deacetylation has increased enormously from their discovery. The importance of these proteins in the regulation of metabolism is increasing since they are involved in different key aspects of cell physiology from cell cycle control to metabolism and antioxidant protection (Santa-Cruz Calvo et al., 2012).

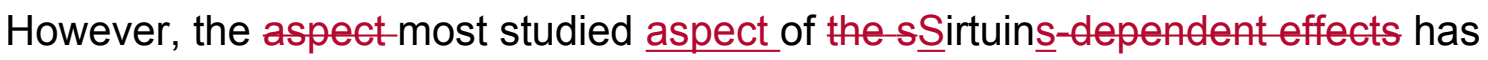
been their role in effects on-longevity (Guarente, 2011, 2013). In mammals, CR induces the expression and the activity of sirtuins in many organs and their activity activities has have been associated with most of the metabolic effects found in these organisms afterproduced by CR (Imai and Guarente, 2010). For example, SIRT1 has been suggested to play an important role since this enzyme activates eNOS by deacetylation in human vascular cells (Mattagajasingh et al., 2007). Further, it has been demonstrated that eNOS is 
induced by CR in mice and human muscle (Civitarese et al., 2007; Nisoli et al., 2005) being essential in the CR-induced mitochondrial biogenesis since in eNOS ${ }^{-/}$mice this induction is blocked (Nisoli et al., 2005).

The peroxisome proliferator-activated receptor gamma coactivator $1 \alpha(\mathrm{PGC} 1 \alpha)$ is the central factor involved in the regulation of mitochondrial mass and the adaptation to energy demand since this transcriptional coactivator stimulates mitochondrial biogenesis, regulates mitochondrial dynamics, modulates oxidative phosphorylation and controls mitochondrial genome copy number (Gouspillou et al., 2014; Handschin and Spiegelman, 2006). Importantly, AMPK and SIRT1, the two mainmajor metabolic sensors in cells, modulate directly are direct modulators of PGC1 1 activity through phosphorylation and deacetylation respectively (Jager et al., 2007; Nemoto et al., 2005). Thus, SIRT1 and AMPK modulating-modulate PGC1 $\alpha$ activity at the transcriptional and post-translational level (Martin-Montalvo and de Cabo, 2013) (Fig. 1).

Into the sirtuins family, SIRT3, a member located in the mitochondria, seems to be very importanta very important role in the regulation of mitochondrial activity especially in fat metabolism (Kendrick et al., 2011). SIRT3 levels increase in skeletal muscle after CR and decrease under high-fat diet feeding indicating a regulation depending on the calorie intake (Palacios et al., 2009). SIRT3 has been also involved in the regulation by deacetylation of electron transport chain complex II activity (Kendrick et al., 2011), modulation of the activity of Mn-SOD (Qiu et al., 2010) or regulation of autophagy by deacetylation of FOXO3 (Kume et al., 2010). Furthermore, SIRT3 KO mice show repression of PGC1 $\alpha$ down regulation and low levels of AMPK phosphorylation after CR indicating a key 
role of this mitochondrial sirtuin in metabolic adaptation to calorie intake (Palacios et al., 2009).

Interestingly, these mechanisms regulate each other in all the organisms studied in an evolutionary conserved response that avoids fruitless energy expenditure and use recycled structures to maintain organisms' survival.

\section{Regulation of AMPK and mTOR controls energy metabolism.}

The $\epsilon$ Control of mitochondrial biogenesis and turnover through AMPK and mTOR activities is key in the regulation cell metabolism and, in particular, mitochondria. AMPK stimulates cellular catabolism of sugars, proteins and lipids. AMPK inhibits by phosphorylation several anabolic enzymes such as acetyl-coenzyme A (CoA) carboxylase (ACC), glycerol phosphate acyl transferase (GAPT), 3-hydroxyl-3-methylglutaryl CoA reductase (HMGCR) and glycogen synthase (GS) affecting glycogen and lipid biosynthesis (Hardie et al., 2012). At the same time, AMPK also inhibits the transcriptional activity of sterol regulatory element binding protein (SREBP) decreasing the expression of genes involved in fatty acid synthesis (Li et al., 2011). But, more importantly, AMPK also-inhibits mTORC1 activity both directly (Gwinn et al., 2008) or indirectly (Inoki et al., 2003).

One of the most important aspects of AMPK and mTORC1 regulation is their contrary effect on autophagy and mitophagy. Whereas mTORC1 negatively regulates autophagy through phosphorylating the mediators, unc-51-like kinase 1/2 (ULK-1 and ULK-2), in inhibitory sites (Chan, 2009; Kim et al., 2011), AMPK positively regulates autophagy by phosphorylating these proteins in their 
activating sites (Alers et al., 2012). In autophagy, The regulation of Forkhead box proteins (FoxO) plays an essentialalso an important role in mito- and autophagy. These proteins are a family of transcription factors playing key roles in the regulation of cell growth, proliferation, differentiation and longevity. FoxO are involved in the induction of several stress response genes (Goto and Takano, 2009) highlighting their importance in the induction of protective mechanisms in the organisms. In fact, the regulation of $\mathrm{FoxO}$ proteins during ageing and their modulation by metabolic regulators such as mTOR, AMPK and sirtuins have associated these proteins with the protective effect of CR, polyphenols or exercise against mitochondrial damage through by regulating autophagy and antioxidant protection preventing by these mechanisms the progression of ageing-related disease (Lopez-Lluch and Navas, 2015)_(LopezLluch and Navas, 2016) (Fig. 1).

\section{Mitochondrial activity and turnover are affected during ageing.}

Mitochondria play a key role in the pathophysiology of ageing and in earlier stages of the events leading to the ageing phenotype (Gonzalez-Freire et al., 2015). Some evidence indicate that ageing is associated with the accumulation of deficient mitochondria that produce a significant increase of ROS generation (Chistiakov et al., 2014; Merry, 2002). In human muscle, ageing is accompanied by a higher need of mitochondria to maintain the same respiratory capacity as in young people indicating decay in mitochondrial efficiency (Larsen et al., 2012). Although the role of mitochondria as the mainmajor source of intracellular ROS production in ageing has been questioned during the last 
years (Gram et al., 2015; Payne and Chinnery, 2015), most data suggest that there is an increase in oxidative damage in the elderly affecting senescence (Lu and Finkel, 2008), fertility declining (Benkhalifa et al., 2014), skin ageing (Kammeyer and Luiten, 2015), neutrophils' activity (Kovalenko et al., 2014) or skeletal muscle (McDonagh, 2016).

Due to the dual role of ROS as damaging and signalling factors, a moderate level of ROS production during life can be beneficial by inducing hormetic responses that reinforce the capacity of the organism to respond to stronger injury (Rattan, 2008). In model organisms, a recent study in Drosophila indicates that ROS increase with age as mitochondrial function deteriorates but high levels of ROS during the life of the organism seems to act as secondary messengers inducing endogenous protective mechanisms in the animals that can increase lifespan (Scialo et al., 2016). If ROS production is balanced with antioxidant enzymes, oxidative damage did not occur, but if these enzymes decay during aging, as has been found in different models (Rodriguez-Bies et al., 2015; Rodriguez-Bies et al., 2016; Tung et al., 2014; Tung et al., 2015b), oxidative damage accumulates.

In any case, evidence indicate that damaged mitochondria show low efficiency producing lower ATP and higher release of ROS through a deficient activity of the mitochondrial electron transport chain complexes. Near all the complexes of the mitochondrial electron transport chain are involved in ROS production and it has been recently shown that, the assembly of these complexes in supercomplexes seems to regulate ROS production (Goncalves et al., 2015). Thus, a progressive deterioration of supercomplexes in-during ageing could be 
the cause of an irreversible increase in ROS during ageingin older organisms (Genova and Lenaz, 2015).

Accumulation of damaged mitochondria during ageing is also associated with the decline of the mitochondrial turnover through inhibiting mitophagy; a specific autophagy process that removes damaged mitochondria (Chistiakov et al., 2014). The correct activity of the quality control of mitochondria is considered a crucial factor in counteracting ageing process (Weber and Reichert, 2010). Furthermore, $r$ Renovation of the mitochondrial network plays a key role in health-span increase after CR (Lopez-Lluch et al., 2008) indicating that the control of the accumulation of damaged mitochondria is an important factor in the progression of ageing. In fact, prolongevity factors such as CR, exercise and polyphenols prevent the accumulation of damaged mitochondria at least in part by inducing mito- and autophagy (Lopez-Lluch and Navas, 2015)_(LopezLluch and Navas, 2016) (Fig. 2).

Another factor involved in the decay in mitochondrial turnover during ageing is the impairment of mitochondrial biogenesis, especially in high energy demanding tissues such as muscle, brain or heart (Conley et al., 2000; Short et al., 2005). As in the case of induction of autophagy, ageing delaying interventions such as CR, exercise or nutraceuticals as resveratrol also induce mitochondrial biogenesis in humans and other organisms (Baur et al., 2010; Feige et al., 2008; Rodriguez-Bies et al., 2015). Thus, it seems clear that maintenance of mitochondrial turnover removing ROS-producing damaged mitochondria at the same time that biogenesis produces new components is essential in the prolongevity effects of these factors. 
The bioenergetic state of the cell is directly related with the regulation of mitochondrial dynamics-; the balance in fusion/fission processes occurring in the mitochondrial network. Mitochondrial fusion is associated with optimized mitochondria whereas fission is associated with damaged mitochondria and their removal by mito- or autophagy (Lopez-Lluch et al., 2008). Mitochondrial dynamics are regulated by a group of proteins that regulate fusion and fission in mitochondria. The mainmajor proteins involved in fusion are mitofusins 1 and 2 (Mfn1 and Mfn2) and autosomal dominant optic atrophy-1 (OPA1) (Wai and Langer, 2016). All these proteins are large GTPases localized in mitochondria. Mitofusins are located at the outer membrane of mitochondria acting in the fusion-process of these membranes whereas OPA1 is located in the intermembrane space and acts in the fusion of the inner membranes. Mfn2 is also associated in the connection between mitochondria and the endoplasmic reticulum (ER) playing a role in the development of endoplasmic reticulum (ER) stress and fat accumulation in conditions of metabolic stress (de Brito and Scorrano, 2008, 2009).

Fission segregates components of the network that are irreversibly damaged or unnecessary for subsequent removal (Twig et al., 2008). The mainmajor proteins involved in mitochondrial fission are dynamin related protein 1 (DRP1) and fission protein 1 (FIS1). DRP1 is also a GTPase protein located mainly in the cytosol although a fraction is localized to punctate on mitochondria (Smirnova et al., 2001). DRP1 is recruited on the outer mitochondrial membrane by FIS1 which is already inserted in this membrane (Santel and Frank, 2008; Yoon et al., 2003). However, other factors such as mitochondrial fission factor (Mff) or mitochondrial dynamics proteins of 49 and $51 \mathrm{kDa}$ (MiD49 and MiD51) 
can also mediate DRP1 recruitment in absence of FIS1 (Loson et al., 2013; Shen et al., 2014), although mutations in FIS1 disrupt downstream mitochondrial degradation events indicating the importance of this protein in mitochondrial turnover (Shen et al., 2014).

During ageing, the equilibrium of fusion and fission is disturbed producing the accumulation of damaged mitochondria. Blocking the fission process by depleting FIS1 or blocking DRP1 activity leads to cell senescence in human cells accompanied by the accumulation of damaged mitochondria showing lower mitochondrial potential and higher ROS production (Lee et al., 2007; Park et al., 2010). In fact, senescence has been associated with the accumulation of giant dysfunctional mitochondria characterized by highly interconnected networks and ultrastructural abnormalities in human lymphocytes and mice cells (Beregi and Regius, 1987) accompanied by higher production of ROS and lower mitochondrial respiratory activity (Yoon et al., 2006). These facts highlight the importance of a functional fission machinery to discard damaged mitochondria by autophagy or mitophagy (Gomes and Scorrano, 2008; Twig et al., 2008).

It is not clear, how different morphologies affect mitochondrial function, although changes in mitochondrial shape during development suggest a close relationship between morphology and function. Placenta, skeletal muscle, vascular smooth cells and neurons suffer mitochondrial defects by deficiencies in mitochondrial dynamics (Chen and Chan, 2005). Further, RNAi studies have demonstrated an inverse relationship between fusion capacity and respiration rates mainly affecting complexes I, III and IV in mammals and humans (Chen et al., 2005). This effect is-was reversed by restoring Mfn and OPA1 proteins and 
was not a consequence of changes in biogenesis regulators such as PGC1a, indicating a direct relationship of mitochondrial dynamics and regulation of OXPHOS subunits activities (Chen et al., 2005). In other organs, defects in mitochondrial fusion/fission dynamics are also essential for the quality control of mitochondria in many age-related diseases as in the case of cardiac homeostasis and senescence (Biala et al., 2015), or in neurodegeneration and hypothalamic dysfunction (Zorzano and Claret, 2015). On the other hand, it has been recently proposed that a decrease in the levels of mitochondrial fission proteins can be an adaptive response to increase the resistance against oxidative stress promoting a resistance phenotype in response to a higher ROS production by mitochondrial dysfunction (Mai et al., 2010) or even a defensive response to maintain functional cell mitochondria (Chen and Chan, 2005). Thus, the decrease of fission process would allow mitochondria to escape autophagic degradation and then, to maintainmaintaining enough ATP production even with deficient mitochondria (Gomes and Scorrano, 2011). All these evidence found in mammals models and humans indicate that dysfunction in the control of mitochondrial dynamics can be associated with the accumulation of damaged mitochondria during ageing in different organs including adipose tissue.

Accumulation of damaged mitochondria during ageing-or by high-fat diet increases the production of ROS that can not only produce damage in molecules and structures but also modulate cell responses such as antioxidant response, removal of damaged molecules, induction of organelle turnover and regulation of mitochondrial activity (Ristow, 2014). This means that the balance between ROS production and the capacity of the cells to respond to ROS levels 
could affect cell homeostasis during ageing. In fact, , LEow levels of ROS have been also involved in the modulation of glucose metabolism and the increase in oxidative metabolism and stress resistance in CR conditions or after exercise indicating a role of ROS in the regulation of metabolism (Barbieri et al., 2013). Moreover, in a model of longevity in Drosophila it has been recently demonstrated that moderated levels of ROS can induce prolongevity effects (Scialo et al., 2016) by activating recycling mechanisms such as mitophagy and autophagy (Weber and Reichert, 2010). Although the ROS-dependent mechanisms involved in the increase of mitochondrial metabolism have to be clarified, it seems that the AMPK-dependent modulation of FoxO-dependent autophagy and PGC1a-regulated mitochondrial biogenesis are involved (Li et al., 2015). In resume, a moderate production of ROS accompanied by the maintenance of the capacity to respond to them is enough to maintain the population of mitochondria in a balanced status, creating new mitochondria and eliminating damaged components.

Mitochondrial turnover can be regulated by different factors during ageing.

Control of mitochondrial dynamics including turnover seems to play an essential role in ageing. Multiple factors affect mitochondrial activity and many of them have been associated with cell senescence and ageing as has been recently reviewed (Ziegler et al., 2015). Control of mitochondrial dynamics including turnover seems to play an essential role in ageing. Unfortunately the factors 
that control the change in mitochondrial dynamics during ageing have not been determined yet (Collins et al., 2002). The identification of these factors will be essential to understand if they are dysregulated during ageing or in pathological situations. Among them PGC1 $\beta$ can be one of the candidates since this transcription factor control basal mitochondrial biogenesis and stimulates mitochondrial fusion through the modulation of Mfn2 (Liesa et al., 2008) (Fig. 2).

Another interesting candidate is the mitochondrial E3-ubiquitin ligase 1 (Mul1), a mitochondrial membrane protein showing different functions (Peng et al., 2016). Mul1 acts as an ubiquitin-ligase that ubiquitinate proteins such as Mfn2, p53 or ULK1, leading to their degradation. Further, Mul1 also acts as small ubiquitin-like modifier (SUMO) E3-ligase, sumoylating other proteins such as DEP1-DRP1 enhancing its stabilization. Then, Mul1 controls mitochondrial dynamics by inducing fission at the same time that reduce fusion by inducing Mfn2 degradation (Peng et al., 2016). In fact, Mul1 has been associated with the fragmentation, depolarization and clearance of mitochondria through mitophagy in a mechanism regulated by FoxO (Lokireddy et al., 2012). Furthermore, the activity of this ubiquitin ligase has been recently associated with the maintenance of muscle mass (Palus et al., 2014).

Further, SIRT3 also emerges as an important factor in the control of mitochondrial dynamics during ageing since it deacetylates OPA1 elevating its GTPase activity and then, increasing fusion processes (Samant et al., 2014). For further information, this mechanism and others have been recently reviewed in deep in its role in ageing and ageing-related diseases (Benigni et al., 2016). 
Recent research has found another component in the control of cell metabolism and mitochondrial activity, sestrins. Sestrins are another highly conserved protein family encoded by genes known as Sens that are upregulated after environmental stress, hypoxia and DNA damage (Budanov and Karin, 2008). Whereas invertebrates' genome contains only a single sens gene, vertebrates express three sestrins (Sens1-3). Their importance in metabolism regulation resides in their regulation of mTORC1 activity. Sestrins are able to bind AMPK activating it and, by this mechanism, repress mTORC1 activity (Budanov and Karin, 2008). The prolongevity effect of sestrins have been recently proposed after the experiments performed in dSens-deficient D. melanogaster (Lee et al., 2010) and in C. elegans (Yang et al., 2013). In mice, these proteins have also been associated with the regulation of metabolism producing a prolongevity effect (Lee et al., 2012).

If sestrins expression decays during ageing, accumulation of damaged mitochondria will increase due to reduction of mito- or autophagy. In agreement with this hypothesis, it has been recently demonstrated that the protective effect of sestrins depends on the promotion of autophagic degradation of Keaps 1 and Nrf2 activation by inhibition of mTORC1 (Rhee and Bae, 2015) probably by eliminating damaged mitochondria as in the case of rat kidney cells (Ishihara et al., 2013). The same mechanism has been associated with the protective effect of sestrin2 in rodent dopaminergic neurons against rotenone toxicity (Hou et al., 2015). Future research about these proteins and its role in the control of metabolism in muscle, adipose tissue and liver during ageing will clarify their importance in the accumulation of fat, especially in humans (Fig.2). 
Interestingly, other factors, such as mitochondrial signaling peptides have been recently described to mitochondrial homeostasis. Defects in mitochondrial activity activate a retrograde response that communicat mitochondrial dysfunction to nucleus through activation of AMPK/PGC1a signalling axis (Quiros et al., 2016). The short open reading frame found within the mitochondrial 12S rRNA known as MOTS-C (mitochondrial open reading frame of the 12S rRNA-c) appears mainly in skeletal muscle and activate AMPK (Lee et al., 2016). Interestingly, treatment with MOTS-C in mice prevent agedependent and high-fat diet induced insulin resistance and obesity indicating an important role of mitochondria in the control of fat metabolism probably producing prolongevity effects (Fuku et al., 2015; Lee et al., 2015). Other mitochondrial-derived peptide is humanin, a 24 aminoacid peptifde encoded within the 16S ribosomal RNA gene (Capt et al., 2016), this peptide has been proposed to produce strong cytoprotective actions against various stress and diseases (Lee et al., 2013a). Humanin has demonstrated protective capacity in age-related diseases such as T2DM and Alzheimer's disease (Mahboobi et al., 2014) and is able to restore mitochondrial function after oxidative injury in retinal pigment epithelial cells (Sreekumar et al., 2016). These peptides open a new field in the study of regulatory mechanisms involved in the regulation of mitochondrial homeostasis associated with ageing and bioenergetics.

\section{Mitochondrial dysfunction is associated with age-dependent fat accumulation.}


It seems clear that mitochondrial dysfunction is associated with ageing progression. A considerable body of evidence demonstrate that mitochondrial dysfunction also plays an important role in obesity and obesity-related diseases such as insulin resistance and fatty liver. Impaired mitochondrial function and dynamics have been associated with diet induced obesity mainly affecting liver and skeletal muscle (Putti et al., 2015). These dysfunctions have beenhave been regulated-modulated by CR and exercise (Putti et al., 2015). Further, induction of mitochondrial dysfunction by blocking respiration or knocking down the mitochondrial transcription factor $A$ induce insulin resistance in human adipocytes and affects the secretion of adiponectin in an effect attributed to the increase in ROS production by defective mitochondria (Wang et al., 2013).

Regulators of mitochondrial activity and turnover such as sirtuins have been associated with the biogenesis of adipocytes. Very recently, a key role of SIRT1 in hyperplasia, the increase of cell number, of adipocytes, has been suggested. Interestingly, hyperplasia has been also associated with dysregulation of metabolism - in adipocytes and enhanced differentiation in adipocytes (Abdesselem et al., 2016). This indicates that SIRT1 is needed for a correct regulation of the adipose tissue function (Abdesselem et al., 2016).

In relationship with mitochondrial dynamics, changes in the mitochondrial network have been associated with obesity-related diseases. Apart of the effects due to defects in Mfn1 and 2 found in neurons, skeletal muscle or monocytes-by defects in Mfn1 and 2, a screen of differentially expressed genes from skeletal muscle of obese rats has identified Mfn2 as a suppressor of obesity (Bach et al., 2003). Further, conditions that increase energy expenditure such as cold exposure, chronic exercise or nutraceuticals as resveratrol are 
associated with higher Mfn2 expression in skeletal muscle and brown adipose tissue in mice and humans (Cartoni et al., 2005; Dolinsky et al., 2013; Soriano et al., 2006). Interestingly, these conditions are known to induce SIRT1 that has been recently shown associated with the suppression ofto suppress mitochondrial dysfunction in a mouse model of ischemia by modulating Mfn2 activity by deacetylation indicating a connection between SIRT1 and mitochondrial dynamics (Biel et al., 2016). On the other hand, higher mitochondrial fission has been also associated with fat accumulation in genetically induced obesity (ob/ob) mice or in high-fat induced obesity (Jheng et al., 2012) and with insulin resistance in the same mice model (Holmstrom et al., 2012).

Prolongevity $\mathrm{CR}$ mimetics such as rapamycin and metformin have also been proposed to modulate mitochondrial dynamics in obesity and age-related diseases (Mercken et al., 2012). Although rapamycin has been associated with the prevention of age-related diseases and the extension of lifespan in both, obese and lean mice (Liu et al., 2014) and to avoid hyperlipidemia and diabetes-like syndrome in aged obese rats (Scarpace et al., 2016), a recent paper indicate that rapamycin reduces lifespan in the obese $\mathrm{db} / \mathrm{db}$ mice (Sataranatarajan et al., 2016). In the case of metformin, it has been associated with the prevention of sedentarism-dependent damages (Senesi et al., 2016). Further, metformin-dependent AMPK activation has recently demonstrated protection of mitochondria in huntington's disease by affecting mitochondrial dynamics (Jin et al., 2016). These results demonstrate that these pharmaceuticals compouds are promising in modulation of mitochondrial activity 
in ageing and obesity although more studies are needed to demonstrate their effectivity in mammals and humans.

- In T2DM and obese people abnormalities due to lower mitochondrial functionality have been shown. Interestingly, in these cases a transient increase in oxidative capacity was found in human liver at the same time that oxidative stress increases (Koliaki and Roden, 2016). These changes have been associated with the increase in mitochondrial fragmentation and down regulation of fusion-regulatory factors associated with impaired bioenergetics in human muscle (Bach et al., 2005). Reduced expression of Mfn2 has been found in skeletal muscle in both, obese and T2DM patients (Bach et al., 2005). Lower levels of Mfn2 have been associated with the fragmentation of the mitochondrial network and the decrease of membrane potential in rat skeletal muscle (Pich et al., 2005). This reduction is directly proportional to BMI and inversely proportional to insulin response (Pich et al., 2005). Mfn2 depleted rat muscle cells show a reduced proton leak and lower bioenergetics efficiency indicated by lower glucose oxidation and mitochondrial respiration (Bach et al., 2003). This lower bioenergetics efficiency due to Mfn2 loss of function has been associated with the development of obesity by reducing energy expenditure and increasing fat energy store in mammals and humans (Liesa et al., 2009). On the other hand, physical exercise increases Mfn1 and 2 in human skeletal muscle indicating the importance of mitochondrial dynamics in the bioenergetic response of mitochondria to energy requirements (Cartoni et al., 2005).

Recently, pProhibitin has been recently- linked to the regulation of mitochondrial activity and the development of obesity. Prohibitin is a complex of two proteins, PHB-1 and PHB-2, that has been associated with longevity in studies carried 
out in worms (Lourenco et al., 2015). Prohibitins are ubiquitous, evolutionary conserved proteins that form a ring-like complex at the inner membrane of the mitochondria (Artal-Sanz and Tavernarakis, 2009). They have been associated with mitochondrial fusion by stabilizing OPA1 that mediates inner membrane fusion and cristae morphogenesis. However, the exact function of this complex in mitochondria is not completely clear although it has been associated with mitochondrial function, fat metabolism and oxidative stress response in mice (Theiss et al., 2009). Interestingly, overexpression of prohibitin in adipocytes produces the development of obesity in mice accompanied by the induction of mitochondrial biogenesis (Ande et al., 2014). This effect contrasts with the higher mitochondrial fission associated with fat accumulation in obese mice (Jheng et al., 2012). On the other hand, downregulation of PHB by microRNA$27 \mathrm{a}$ and $27 \mathrm{~b}$ resulted in impaired mitochondrial biogenesis and structure integrity, lower activity of complex I and higher ROS production in adiposederived stem cells (Kang et al., 2013). Moreover, inactivation of PHB-2 in mice brain affects mitochondrial structure and activity resulting in neurodegeneration by hyperphosphorylation of Tau (Merkwirth et al., 2012) or alters beta-cell function in pancreas producing diabetes (Supale et al., 2013). All these facts highlight the importance of a balanced mitochondrial dynamics in the physiology adipose and other tissues and the complex regulation of the different factors involved in mitochondrial fusion and fission. Further studies are needed to understand how this balance is controlled and how ageing can affect it producing a cascade of events leading to the age-related diseases associated with fat accumulation. 
$\underline{\text { Regarding sestrins, transgenic mice deficient in Sens2 and Sens } 3 \text { genes have }}$ demonstrated the important role of these proteins in the suppression of age and obesity-associated mitochondrial disorders (Bae et al., 2013; Lee et al., 2012). Sesn2 KO mice show glucose intolerance, lower insulin response and development of hepatosteatosis (Lee et al., 2013b; Lee et al., 2012). On the other hand, Sens3 has been recently associated with the activation of mTORC2 and Akt phosphorylation enhancing insulin sensitivity and glucose metabolism in mice (Tao et al., 2015). It has been suggested that hypernutrition and lack of exercise can induce chronic mTORC1 activation that is accompanied by Sesn2 up-regulation in order to control the excessive activity of mTORC1 (Lee et al., 2010). Other studies carried out in rats proposed that sestrins-dependent mTORC1 inactivation through AMPK induction is critical in the maintenance of autophagy and, then, the elimination of dysfunctional mitochondria (Ishihara et al., 2013). Interestingly, sestrins are also regulated by oxidative stress through p53, NRF-2, AP-1 and FOXO and they regulate several components that reduce ROS production, increase mitochondrial biogenesis and induce mitophagy increasing the efficiency of oxidative metabolism in mice (Lee et al., 2013b).

All these evidences suggest that sestrins could be considered key factors in the control of mitochondrial homeostasis in ageing and obesity and probably T2DM. Research performed in invertebrates demonstrate that the inactivation of sestrins results in metabolic pathologies that include oxidative damage, mitochondrial dysfunction and fat accumulation (Lee et al., 2013b). However, to date there is no information available about the activity of sestrins in adipose tissue and its behaviour during ageing. Indirect evidences indicate a putative 
role of this family of proteins in fat accumulation. Resveratrol (RSV), a polyphenol that prevents lipid accumulation in liver, blocks Liver $\mathrm{X}$ receptor (LXRa)-mediated hepatic lipogenesis by inducing Sens2 gene (Jin et al., 2013), indicating a putative role of this family of proteins in fat accumulation. LXRa is a transcription factor that modulates lipid synthesis in liver (Joseph et al., 2002) and regulates insulin-stimulated lipogenesis (Chen et al., 2004). LXRa induces the expression of fatty acid synthesis (FAS), acetyl-CoA carboxylase (ACC) and sterol regulatory element binding protein 1c (SREBP-1c) (Grefhorst et al., 2002; Repa et al., 2000). Therefore, the Sens2-dependent repression of LXRa induced by RSV would indicate a key role of these proteins in fat accumulation during ageing.

Do sestrins affect fat accumulation and modulate mitochondrial activity and turnover during ageing? No information is available to date. However, the above indicated evidences point to these proteins as putative key regulators. In fact, although no studies have been performed yet in humans, the activity of sestrins has been recently suggested in the effect of exercise to prevent sarcopenia, frailty and obesity in the elderly (Sanchis-Gomar, 2013).

All these reports indicate that there is a downregulation of fusion in mitochondria in obesity related diseases. When fat accumulates, mitochondrial activity can decrease by increasing fission processes and accumulating then, low potential small mitochondria. This response is opposite to the behaviour found during ageing in which mitochondrial network tends to increase by inducing fusion in a response that probably is triggered to maintain the capacity 
of mitochondria (Fig. 2). It is probable that the incapacity to control mitochondrial dynamics during ageing, when fat accumulates in WAT, liver or muscle, produces the accumulation of damaged mitochondria and the resulting consequences as metabolism unbalance, insulin resistance or oxidative stress.

Role of sestrins in mitochondrial regulation. A role in ageing-dependent fat accumulation?

Recent research has found another component in the control of cell metabolism and mitochondrial activity, sestrins. Sestrins are another highly conserved protein family encoded by genes known as Sens that are upregulated after environmental stress, hypoxia and DNA damage (Budanov and Karin, 2008). Whereas invertebrates' genome contains only a single sens gene, vertebrates express three sestrins (Sens1-3). Their importance in metabolism regulation resides in their regulation of mTORC1 activity. Sestrins are able to bind AMPK activating it and, by this mechanism, repress mTORC1 activity (Budanov and Karin, 2008).

The prolongevity effect of sestrins have been recently proposed after the experiments performed in dSens-deficient D. melanogaster (Lee et al., 2010) and in C. elegans (Yang et al., 2013). In mice, these proteins have been associated with the regulation of metabolism producing a prolongevity effect. Transgenic mice deficient in Sens 2 and Sens 3 genes have demonstrated the important role of these proteins in the suppression of age and obesityassociated mitochondrial disorders (Bae et al., 2013; Lee et al., 2012). Sesn2 KO mice show glucose intolerance, lower insulin response and development of 
hepatosteatosis (Lee et al., 2013b; Lee et al., 2012).On the other hand, Sens3 has been recently associated with the activation of mTORC2 and Akt phosphorylation enhancing insulin sensitivity and glucose metabolism in mice (Tao et al., 2015). It has been suggested that hypernutrition and lack of exercise can induce chronic mTORC1 activation that is accompanied by Sesn2 up-regulation in order to control the excessive activity of mTORC1 (Lee et al., 2010). On the other hand, other studies carried out in rats proposed that sestrins-dependent mTORC1 inactivation through AMPK induction is critical in the maintenance of autophagy and, then, the elimination of dysfunctional mitochondria (Ishihara et al., 2013). Interestingly, sestrins are also regulated by exidative stress through $\mathrm{p53}, \mathrm{NRF}-2, \mathrm{AP}-1$ and FOXO and they regulate several components that reduce ROS production, increase mitochondrial biogenesis and induce mitophagy increasing the efficiency of oxidative metabolism in mice (Lee et al., 2013b).

All these evidences suggest that sestrins could be considered key factors in the control of mitochondrial homeostasis in ageing and obesity and probably T2DM. Research performed in invertebrates demonstrate that the inactivation of sestrins results in metabolic pathologies that include oxidative damage, mitochondrial dysfunction and fat accumulation (Lee et al., 2013b). However, to date there is no information available about the activity of sestrins in adipose tissue and its behaviour during ageing. Indirect evidences indicate a putative role of this family of proteins in fat accumulation. Resveratrol (RSV), a polyphenol that prevents lipid accumulation in liver, blocks Liver $X$ receptor (LXRa)-mediated hepatic lipogenesis by inducing Sens2 gene (Jin et al., 2013), indicate a putative role of this family of proteins in fat accumulation. LXRa is a 
transcription factor that modulates lipid synthesis in liver (Joseph et al., 2002) and regulates insulin-stimulated lipogenesis (Chen et al., 2004). LXRa induces the expression of fatty acid synthesis (FAS), acetyl-CoA carboxylase (ACC) and sterol regulatory element binding protein 1c (SREBP-1C) (Grefhorst et al., 2002; Repa et al., 2000). Then, the Sens2-dependent repression of LXRa induced by RSV would indicate a key role of these proteins in fat accumulation during ageing.

Do sestrins affect fat accumulation and modulate mitochondrial activity and turnover during ageing? No information is available to date. However, the above indicated evidences point to these proteins as putative key regulators. In fact, although no studies have been performed yet in humans, the activity of sestrins has been recently suggested in the effect of exercise to prevent sarcopenia, frailty and obesity in the elderly (Sanchis-Gomar, 2013). If sestrins expression decays during ageing, accumulation of damaged mitochondria will increase due to reduction of mito- or autophagy. In agreement with this hypothesis, it has been recently demonstrated that the protective effect of sestrins depends on the promotion of autophagic degradation of Keaps 1 and Nrf2 activation by inhibition of mTORC1 (Rhee and Bae, 2015) probably by eliminating damaged mitochondria as in the case of rat kidney cells (Ishihara et al., 2013). The same mechanism has been associated with the protective effect of sestrin 2 in rodent dopaminergic neurons against rotenone toxicity (Hou et al., 2015). Future research about these proteins and its role in the control of metabolism in muscle, adipose tissue and liver during ageing will clarify their importance in the accumulation of fat, especially in humans (Fig.2). 


\section{Metabolic syndrome, fat accumulation and mitochondria.}

Obesity and T2DM are considered age-related diseases. In several of the models studied including rodents and humans, these diseases are associated with mitochondrial dysfunction indicated by low expression of mtDNA and reduction of the levels of proteins involved in oxidative phosphorylation (OXPHOS). Thus, mitochondrial dysfunction has been described in the main organs involved in the development of metabolic syndrome during ageing such as muscle, liver and adipose tissue (Patti and Corvera, 2010).

The increase in fat mass is one of the major determinants of insulin resistance (Larson-Meyer et al., 2006). In humans, it has been shown that insulin resistance is responsible of the increase of lipids in liver and skeletal muscle and the decrease of the capacity of adipose tissue to store lipids (Toledo and Goodpaster, 2013). On the other hand, deficiency in mitochondrial activity in muscle is associated with obesity and T2DM. These mitochondrial deficiencies include reduction of fatty acid oxidation, lower expression of genes involved in oxidative activity and decrease of electron transport chain activities (Mootha et al., 2003; Patti and Corvera, 2010; Simoneau et al., 1999; Toledo and Goodpaster, 2013). Further, alterations in mitochondrial dynamics and in particular in Mfn2, have been associated with a reduced mitochondrial function in skeletal muscle in obesity and T2DM (Zorzano et al., 2009) and recently aberrant mitochondrial fission has been also associated with insulin resistance in mouse skeletal muscle (Jheng et al., 2012). The deficiency in the capacity of mitochondria to metabolize lipids has been proposed to predispose WAT and skeletal muscle to accumulate lipids and then, to the impairment-development of insulin resistance (Lowell and Shulman, 2005). However, other studies 
performed in rats suggested that insulin resistance is accompanied by an increase in mitochondrial content and an increase in oxidation of lipids (Dela and Helge, 2013; Garcia-Roves et al., 2007; Hancock et al., 2008; Turner et al., 2007). These discrepancies indicate that the role of mitochondria in muscle in high-fat diet induced T2DM is not completely clear. Probably, the accumulation of fat in muscle induces a higher oxidative metabolism based on fatty acids catabolism but this can be accompanied by the increase of mitochondria including damaged and ROS-producing structures that accumulate by a deficient mitochondrial turnover and fusion/fission mechanisms. In fact, it has been shown that, in both, rodents and humans, skeletal muscle from high-fat fed conditions show higher $\mathrm{H}_{2} \mathrm{O}_{2}$-mitochondrial production affecting the redox state of the cells but without changes in the mitochondrial respiratory function (Anderson et al., 2009). Further experiments performed in rats demonstrated that exposure to prepregnancy maternal obesity impairs the activity of factors involved in mitochondrial dynamics in the offspring (Borengasser et al., 2014). The accumulation of damaged mitochondria would explain the increase of ROS in skeletal muscle associated with insulin resistance of obese individuals and the appearance of T2DM and muscle malfunction during normal ageing as results of the deteriorated physiology of the organs (Barbieri et al., 2013). In fact, the elucidation of the mechanisms affecting mitochondrial dynamics in skeletal muscle from obese people has been suggested to be essential to understand muscle insulin resistance and to find therapeutic targets (Jheng et al., 2015).

On the other hand, obesity is associated with the impairment of the ability to transit between fatty acid and glucose metabolism. This impairment is known as 
"metabolic inflexibility" (Griffin et al., 2015), that is present in obese people since they are able to oxidize lipids in insulin-stimulated conditions instead to metabolize glucose being mitochondria the key organelles affecting this incapacity (Storlien et al., 2004).

Among the regulators of metabolism, AMPK seems to be the key component in the progression of metabolic syndrome and insulin resistance in WAT. Phosphorylated AMPK modulates the activity of many downstream substrates resulting in the increase in lipid oxidation and the uptake of glucose (Jager et al., 2007). Moreover, deficiency in AMPK results in glucose intolerance, lower physical capacity and obesity; symptoms of metabolic disease (Steinberg et al., 2010). Taken into consideration the central role of AMPK in the control of metabolism and mitochondria (Burkewitz et al., 2014), modification of levels or activity of AMPK could explain the deterioration of adipose tissue physiology during ageing. Regarding this aspect, a new regulator of AMPK, prolyl isomerase, Pin1, has been shown to rise in high fat state in mouse liver (Nakatsu et al., 2011). This increase is associated with the accumulation of fat since in Pin1 KO mice, a high fat diet-induced obesity resistant phenotype has been described (Nakatsu et al., 2015). Interestingly, the deregulation of Pin1 has been also implied in ageing and age-related diseases (Lee et al., 2011). Taken into consideration the plasticity of mitochondria and the complex relationship between the different regulators of mitochondrial dynamics and turnover, the study of the control of these regulators during ageing and fat overloading must be studied in deep to design efficient strategies against ageing-related diseases caused by deficient fat metabolism. 


\section{Inflammation and lipid accumulation.}

Another important fact to be taken into consideration in the accumulation of fat during ageing is the proinflammatory status that accompanies obesity. It is known that visceral fat is an important source of proinflammatory cytokines contributing to insulin resistance. In this process, the activation of different forms of inflammasome is one of the key factors. Nod-like receptors (NLRs) are an evolutionary conserved family of cytoplasmic receptors whose function is to recognize pathogen- and danger-associated molecular patterns (PAMPs and DAMPs). These receptors form multimeric protein complexes that bind to a caspase activation and recruitment domain (CARD) and caspase 1 (Casp-1). After recognizing PAMPs or DAMPs, Casp-1 is activated and catalyses the transformation of proinflammatory cytokines to their mature forms (Lupfer and Kanneganti, 2012). The release of danger signals from mitochondria promotes the formation of inflammasomes. In fact, mitochondria is considered a central regulator of NLRP3 inflammasome activation through $\mathrm{ROS}, \mathrm{Ca}^{2+}$, decrease of $\mathrm{NAD}^{+}$, Mfn or mitochondrial DNA (Gurung et al., 2015). In patients with diabetes, the inhibition of autophagy results in the accumulation of dysfunctional mitochondria leading to higher ROS production and activating inflammasome (Harijith et al., 2014). On the other hand, Casp-1 activates several pathways that precipitate mitochondrial disassembly, increase ROS production and breaks up mitochondrial network, triggering a vicious cycle in which mitochondrial dysfunction activates inflammasome and Casp-1 induces mitochondrial dysfunction (Yu et al., 2014). Prolongevity effectors such as CR or resveratrol would reduce NLRP3-inflammasome activation preventing the activation of this vicious cycle. In fact, we have recently found that NLRP3 
activity increases in mouse liver during ageing but this activity is inhibited by RSV probably by reducing the level of oxidative stress increasing the mitochondrial turnover and eliminating by this mechanism ROS-producing deficient mitochondria (Baur et al., 2006; Tung et al., 2015a).

Recently, a relationship between lipid accumulation in liver and inflammasome complex activity has been suggested. Lipogenesis in liver is modulated by sterol regulatory element binding proteins (SREBPs). These proteins are activated by hepatitis $\mathrm{C}$ virus and induce the accumulation of lipid droplets probably by activation of the NLRP3-inflammasome complex (McRae et al., 2015). Moreover, chronically overactive inflammasome activity and production of IL-1 $\beta$ has been associated with the abnormal lipid accumulation in old rat liver resulting in steatohepatitis (Chung et al., 2015). The activity of inflammasome has been associated with dysregulation of PPARa and SREBP1c (Chung et al., 2015). Furthermore, a NLRP3 KO mice model has demonstrated that the activity of this inflammasome is essential in the development of high-fat dietinduced obesity and in the development of insulin resistance (Stienstra et al., 2011). On the other hand, the increase of the levels of proinflammatory cytokines such as TNF- $\alpha$ and IL-6 found in obesity produce inhibitory effects on Mfn2 expression in human cultured cells (Bach et al., 2005) probably inducing a dysfunction in mitochondrial homeostasis.

These studies suggest that mitochondria can be on the origin of the overactivation of inflammasome in liver and other organs and then, accumulation of fat and development of steatohepatitis. Obesity is, at the same time, responsible of the maintenance of a chronic inflammatory status producing a vicious cycle. Furthermore, the inflammasome activation in fat tissues will 
trigger fat cell senescence, a phenomenon connected with the senescenceassociated secretory phenotype (SASP) (Newsholme and de Bittencourt, 2014). Then, fat accumulation would promote inflammasome associated SASP that would induce cellular senescence in other tissues of the organism aggravating by this mechanism the obesity-dependent chronic inflammation in another vicious cycle (Newsholme and de Bittencourt, 2014) (Fig.3).

\section{Does fat accumulation and mitochondrial dysfunction depend on sexual hormones?}

In humans, fat deposits depend on the gender of the individual (Palmer and Clegg, 2015). Men tend to accumulate visceral fat whereas women accrue more subcutaneous depots prior to menopause but, after menopause, fat depots in women shift to visceral depots (Palmer and Clegg, 2015). The cause of this difference resides in sexual hormones. Oestrogens and their receptors favour lipid accumulation in subcutaneous depot in women but visceral fat deposition in men. The differences in the metabolism of visceral and subcutaneous fat has been related to a higher risk of metabolic syndrome in men than in women due to a higher free fatty acid production in visceral fat than in subcutaneous fat (Palmer and Clegg, 2015). This will explain why women, with higher levels of subcutaneous fat, show less risk for diseases associated with obesity than men that show higher levels of visceral fat deposition. Visceral fat accumulation is associated with many age-related diseases (Huffman and Barzilai, 2009) accompanied by the accumulation of fat in tissues such as the heart, liver and 
skeletal muscle which increases risk for insulin resistance and cardiovascular disease (Kuk et al., 2009).

Some data indicate that oestrogens control the accumulation of fat during ageing. In premenopausal women, oestrogens protect against weight gain by increasing energy expenditure, whereas postmenopausal women gain weight by the natural decrease in endogenous estradiol levels (Gambacciani et al., 1997). These reductions can be prevented by oestrogen replacement therapy, indicating a direct relationship between the levels of these hormones and metabolic control and probably mitochondrial activity (Gambacciani et al., 1997). In men, the decrease in testosterone levels can be responsible of the increase of adiposity and fat accumulation in non-adipose tissue during ageing (Mudali and Dobs, 2004). In a similar response that women, testosterone therapy in men-prevents the gain in visceral fat in non-obese ageing men (Allan et al., 2008).

Another interesting paper has associated the accumulation of fat during ageing with higher levels of follicle-stimulating hormone (FSH) in both men and women (Liu et al., 2015). In this paper, authors indicate that the increase of this hormone not only is responsible for fat accumulation but also affects fat redistribution from subcutaneous and abdominal fat to visceral fat, a fact associated with ageing (Zamboni et al., 2005). The accumulation of fat in heart, liver and skeletal muscle during ageing has been also attributed to the $\mathrm{FSH}$ rise since skeletal muscle and liver also express FSH-receptor (Liu et al., 2015).

Changes in oestrogens levels can also modulate the genetic expression of mitochondrial factors and then, be also responsible of mitochondrial dysfunction 
effect in fat accumulation during ageing (Vasconsuelo et al., 2013). It has been proposed that estradiol and testosterone affect mitochondria during ageing through receptors located even in mitochondria. These oestrogens would directly regulate nuclear DNA-encoded proteins and mitochondrial transcription factors affecting by this mechanism mitochondrial biogenesis (Vasconsuelo et al., 2013). It has been also proposed that mitochondrial genome contains sequences that are similar to those of nuclear hormone-responsive elements (Sekeris, 1990). In fact, these elements have been found in mtDNA accompanied by oestrogen and androgen receptors (Demonacos et al., 1995; Demonacos et al., 1996). The appropriate receptors can interact with these sequences to induce hormone-dependent mitochondrial gene expression (Psarra et al., 2006). In agreement with this, oestrogens and androgens can affect the transcription of mitochondria OXPHOS components (Scheller et al., 2003). Furthermore, tThese hormone-responsible elements are also found in nuclear gene encoding mitochondrial transcription factors that also modulate OXPHOS gene expression (Psarra et al., 2006).

Changes in oestrogens during ageing can also affect directly the activity of regulators of mitochondria. In rats, a model of menopause by using ovariectomization suggested that reduction of oestrogens produced the decrease of PGC1 $\alpha$ expression and the reduction of $\beta$-oxidation in muscle (Cavalcanti-de-Albuquerque et al., 2014). This suggests that an oestrogendependent effect can induce accumulation of fat and probably insulin resistance in muscle.

Despite these reports, a question remains to be answered,answered; can mitochondrial dysfunction be a response to the reduction of sexual hormones 
during ageing? Low levels of oestrogens decrease PGC1a expression indicating a putative decrease in mitochondrial biogenesis (Cavalcanti-deAlbuquerque et al., 2014). Further, mitochondrial dynamics are also regulated by these hormones. In the human breast cancer cell line MCF-7, the mRNA levels of mitochondrial fusion genes increase during estradiol treatment whereas FIS1 gene expression decreases (Sastre-Serra et al., 2012). In these cells, estradiol treatment also induced mitochondrial biogenesis (Sastre-Serra et al., 2012). The same effect was found in other breast cancer cell lines (SastreSerra et al., 2013). Further, in rat models, the expression of prohibitins responds to the levels of oestrogens in WAT. The level of prohibitins were stimulated by estradiol in WAT and liver in both, controls and high-fat fed rats (Choi et al., 2015). Studies performed in other tissues demonstrated that prohibitin genes are induced by oestrogen receptors (He et al., 2011). Taken into consideration the role of all these proteins in the fusion process in mitochondria, it is clear that modifications in the levels of sexual hormones during ageing will affect mitochondrial dynamics and probably affect the accumulation of damaged mitochondria in adipocytes and other fataccumulating organs. The relationship between oestrogen levels, ageing and mitochondrial activity in fat must be further studied (Fig.4).

\section{Mitochondrial function in POMC neurons and its effect on the control of satiety.}

Satiety and hunger is controlled by neuropeptide-Y (NPY), agouti-related protein (Agrp) and pro-opiomelanocortin (POMC) neurons located in the arcuate 
nucleus of the hypothalamus. Both obesity and ageing are associated with rewiring of a principal brain pathway modulating energy homeostasis through promoting reduced activity of satiety pro-opiomelanocortin (POMC) neurons. It has been suggested that the activity of these neurons is importantly affected by mitochondrial dynamics (Nasrallah and Horvath, 2014). In studies performed in mice it has been demonstrated that mitochondrial fusion increases in orexigenic NPY/Agrp neurons inducing neuronal activity that control the storage of excess of energy in fat when mice are fed with high-fat diet (Dietrich et al., 2013). The activity of these neurons is impaired when the mitochondrial fusion mechanism is altered producing resistance to fat gain during high fat diet (Dietrich et al., 2013). However, the depletion of Mfn2 in the anorexigenic POMC neurons causes severe obesity and leptin resistance probably by affecting ER-stress (Schneeberger et al., 2013). Moreover, a dysregulation of the control of mitochondrial activity and turnover could be also associated with dysfunctions in the control of satiety. A recent paper indicates that mTOR activity is elevated in POMC neurons in aged mice. This increase produces cell hypertrophy that the authors associate with obesity-related deterioration of POMC neurons (Yang et al., 2012). On the contrary, the inhibition of mTOR in these neurons caused reduction of food intake and weight loss in old mice indicating the importance of this regulator in satiety control (Yang et al., 2012). These and other results suggest that mitochondrial dynamics in NPY/Agrp and POMC neurons also play an important role in the regulation of the energy balance and in diet and agerelated obesity.

\section{Concluding remarks.}


Many evidences indicate that mitochondrial dysfunction is associated with the development of obesity and the appearance and progression of age-related diseases (Fig.5). Regulators of metabolism such as mTOR, AMPK and sirtuins are involved in this effect and the complex equilibrium between their activities modulates mitochondrial biogenesis, dynamics and turnover. This regulation is affected by sexual hormones that change during ageing and probably the reduction of oestrogens is one of the key factors involved in mitochondrial dysfunction and fat accumulation in humans. During ageing a tendency to increase fusion in mitochondria has been reported in order to maintain mitochondrial activity; however, fat accumulation induces a higher activity of fission mechanisms promoting the accumulation of small and less efficient mitochondria. Probably these two opposite processes end in the accumulation of damaged mitochondria that increase oxidative damage that contribute to the appearance and impairment of insulin resistance, metabolic dysfunction and the release of proinflammatory mediators. In a vicious cycle, insulin resistance and proinflammatory cytokines promote more damage in mitochondria. The study of the response of these metabolic regulators to prolongevity effectors such as $\mathrm{CR}$, polyphenols nutra- and pharmaceuticals or exercise and the role of new modulators such as prohibitins or sestrins in ageing progression will help us to develop therapeutic strategies to delay the damaging effect of fat accumulation during ageing.

\section{Funding.}

The research group is funded by the Andalusian Government grant BIO177 (FEDER funds of European Commission). Research has been funded by the Spanish Ministry of Economy and Competitiveness grant DEP2012-39985. 
Authors are also members of the CIBERER, Instituto Carlos III, of the Spanish Ministry of Health. 


\section{Figure legends.}

Graphical abstract. Mitochondrial homeostasis is basic to maintain a balanced metabolic activity in fat accumulating tissues including adipose tissue, muscle and liver. Ageing and the decay of oestrogens produce an unbalance in mitochondrial homeostasis causing the accumulation of damaged mitochondria as giant or small and damaged mitochondria. Decay of mito- and autophagy processes is also affected. These events produce the reduction of respiration, membrane potential and the increase of reactive oxygen species. Prolongevity factors such as calorie restriction, exercise or several nutraceutical such as polyphenols can reduce mitochondrial dysfunction by maintaining mitochondrial biogenesis, fusion/fission balance and mito- and autophagy during ageing preventing then fat accumulation.

Fig. 1. Molecular mechanisms involved in the maintenance of mitochondrial lipid metabolism. Two main-major systems are involved in the control of metabolism in cells: mTOR and AMPK/SIRT. High fat diets, fat accumulation of T2DM activates mTORC1 that reduces lipid consumption by affecting mitochondrial biogenesis, turnover and dynamics through inhibiting PGC1a. On the other hand, effectors such as CR, exercise of polyphenols activate AMPK/SIRT that inhibit mTORC1 and induce mitochondrial biogenesis through PGC1 $\alpha$ and mitophagy maintaining a mitochondrial balanced activity.

Fig. 2. Mitochondrial dynamics during fat accumulation and ageing. Balanced mitochondrial activity maintains a control of mitochondrial population by fusion/fission balance regulated by Mfn 1 and 2, OPA1 and prohibitins (fusion) and DRP1 and FIS1 (fission) cooperation and the biogenesis of the 
components of mitochondria. A dysfunction in these factors can cause the accumulation of giant mitochondria in ageing or small and damaged mitochondria that increase ROS production by decreasing fusion mechanisms and blocking autophagy processes. Regulation of these processes by AMPK and $\mathrm{mTOR} 1$ regulate these processes.

Fig 3. Role of the inflammasome in fat accumulation during ageing. Mitochondrial dysfunction produces NLRP3 inflammasome activation by increasing ROS that acts as Damage-activating molecular pattern. This activation is associated with cell senescence and SASP and the evolution of hepatosteatosis, obesity and T2DM.

Fig 4. Hormonal control of mitochondrial activity can affect fat accumulation. Age-dependent oestrogen decay will affect mitochondrial dynamics by decreasing mitochondrial fusion and PGC1a activity. This will produce mitochondrial dysfunction. At the same time, the increase of FSH found in aged individuals will contribute to the accumulation of visceral fat that, in a vicious cycle, will contribute to higher mitochondrial dysfunction by inhibiting mito- and autophagy through mTORC1 activity as bas been indicated in Fig. 2 . Visceral fat accumulation is associated with the appearance and impairment of T2DM, evolution of hepatosteatosis and obesity.

Fig. 5. Mitochondrial dysfunction is at the centre of the regulation of fat metabolism during ageing. The balanced activity of mitochondria is essential to control satiety and prevent the appearance and progression of T2DM, obesity and inflammation, hallmarks of age-dependent accumulation of fat. Ageing, high fat diet and sexual hormones decay causes mitochondrial dysfunction whereas 
prolongevity interventions such as calorie restriction, exercise and diet rich in nutraceuticals prevent mitochondrial decay and improve the progression of ageing and fat accumulation during ageing. 


\section{References.}

Abdesselem, H., Madani, A., Hani, A., Al-Noubi, M., Goswami, N., Ben Hamidane, H., Billing, A.M., Pasquier, J., Bonkowski, M.S., Halabi, N., Dalloul, R., Sheriff, M.Z., Mesaeli, N., EIRayess, M., Sinclair, D.A., Graumann, J., Mazloum, N.A., 2016. SIRT1 Limits Adipocyte Hyperplasia through c-Myc Inhibition. J Biol Chem 291, 2119-2135. Doi: 10.1074/jbc.M115.675645.

Abranches, M.V., Oliveira, F.C., Conceicao, L.L., Peluzio, M.D., 2015. Obesity and diabetes: the link between adipose tissue dysfunction and glucose homeostasis. Nutr Res Rev 28, 121-132. Doi: 10.1017/S0954422415000098.

Alers, S., Loffler, A.S., Wesselborg, S., Stork, B., 2012. Role of AMPK-mTORUlk1/2 in the regulation of autophagy: cross talk, shortcuts, and feedbacks. Mol Cell Biol 32, 2-11. Doi: 10.1128/MCB.06159-11.

Allan, C.A., Strauss, B.J., Burger, H.G., Forbes, E.A., McLachlan, R.I., 2008. Testosterone therapy prevents gain in visceral adipose tissue and loss of skeletal muscle in nonobese aging men. J Clin Endocrinol Metab 93, 139-146. Doi: 10.1210/jc.2007-1291.

Ande, S.R., Nguyen, K.H., Padilla-Meier, G.P., Wahida, W., Nyomba, B.L., Mishra, S., 2014. Prohibitin overexpression in adipocytes induces mitochondrial biogenesis, leads to obesity development, and affects glucose homeostasis in a sex-specific manner. Diabetes 63, 3734-3741. Doi: 10.2337/db13-1807.

Anderson, E.J., Lustig, M.E., Boyle, K.E., Woodlief, T.L., Kane, D.A., Lin, C.T., Price, J.W., 3rd, Kang, L., Rabinovitch, P.S., Szeto, H.H., Houmard, J.A., Cortright, R.N., Wasserman, D.H., Neufer, P.D., 2009. Mitochondrial H2O2 emission and cellular redox state link excess fat intake to insulin resistance in both rodents and humans. J Clin Invest 119, 573-581. Doi: 10.1172/JCI37048. 
Artal-Sanz, M., Tavernarakis, N., 2009. Prohibitin couples diapause signalling to mitochondrial metabolism during ageing in C. elegans. Nature 461, 793-797. Doi: $10.1038 /$ nature08466.

Bach, D., Naon, D., Pich, S., Soriano, F.X., Vega, N., Rieusset, J., Laville, M., Guillet, C., Boirie, Y., Wallberg-Henriksson, H., Manco, M., Calvani, M., Castagneto, M., Palacin, M., Mingrone, G., Zierath, J.R., Vidal, H., Zorzano, A., 2005. Expression of Mfn2, the Charcot-Marie-Tooth neuropathy type 2A gene, in human skeletal muscle: effects of type 2 diabetes, obesity, weight loss, and the regulatory role of tumor necrosis factor alpha and interleukin-6. Diabetes 54, 2685-2693. Doi.

Bach, D., Pich, S., Soriano, F.X., Vega, N., Baumgartner, B., Oriola, J., Daugaard, J.R., Lloberas, J., Camps, M., Zierath, J.R., Rabasa-Lhoret, R., Wallberg-Henriksson, H., Laville, M., Palacin, M., Vidal, H., Rivera, F., Brand, M., Zorzano, A., 2003. Mitofusin-2 determines mitochondrial network architecture and mitochondrial metabolism. A novel regulatory mechanism altered in obesity. J Biol Chem 278, 17190-17197. Doi: 10.1074/jbc.M212754200.

Bae, S.H., Sung, S.H., Oh, S.Y., Lim, J.M., Lee, S.K., Park, Y.N., Lee, H.E., Kang, D., Rhee, S.G., 2013. Sestrins activate Nrf2 by promoting p62-dependent autophagic degradation of Keap1 and prevent oxidative liver damage. Cell Metab 17, 73-84. Doi: 10.1016/j.cmet.2012.12.002.

Barbieri, E., Sestili, P., Vallorani, L., Guescini, M., Calcabrini, C., Gioacchini, A.M., Annibalini, G., Lucertini, F., Piccoli, G., Stocchi, V., 2013. Mitohormesis in muscle cells: a morphological, molecular, and proteomic approach. Muscles Ligaments Tendons J 3, 254-266. Doi. 
Baur, J.A., Chen, D., Chini, E.N., Chua, K., Cohen, H.Y., de Cabo, R., Deng, C., Dimmeler, S., Gius, D., Guarente, L.P., Helfand, S.L., Imai, S., Itoh, H., Kadowaki, T., Koya, D., Leeuwenburgh, C., McBurney, M., Nabeshima, Y., Neri, C., Oberdoerffer, P., Pestell, R.G., Rogina, B., Sadoshima, J., Sartorelli, V., Serrano, M., Sinclair, D.A., Steegborn, C., Tatar, M., Tissenbaum, H.A., Tong, Q., Tsubota, K., Vaquero, A., Verdin, E., 2010. Dietary restriction: standing up for sirtuins. Science 329, 1012-1013; author reply 1013-1014. Doi: 10.1126/science.329.5995.1012.

Baur, J.A., Pearson, K.J., Price, N.L., Jamieson, H.A., Lerin, C., Kalra, A., Prabhu, V.V., Allard, J.S., Lopez-Lluch, G., Lewis, K., Pistell, P.J., Poosala, S., Becker, K.G., Boss, O., Gwinn, D., Wang, M., Ramaswamy, S., Fishbein, K.W., Spencer, R.G., Lakatta, E.G., Le Couteur, D., Shaw, R.J., Navas, P., Puigserver, P., Ingram, D.K., de Cabo, R., Sinclair, D.A., 2006. Resveratrol improves health and survival of mice on a high-calorie diet. Nature 444, 337342. Doi: 10.1038/nature05354.

Benigni, A., Perico, L., Macconi, D., 2016. Mitochondrial Dynamics Is Linked to Longevity and Protects from End-Organ Injury: The Emerging Role of Sirtuin 3. Antioxid Redox Signal. Doi: 10.1089/ars.2016.6682.

Benkhalifa, M., Ferreira, Y.J., Chahine, H., Louanjli, N., Miron, P., Merviel, P., Copin, H., 2014. Mitochondria: participation to infertility as source of energy and cause of senescence. Int $J$ Biochem Cell Biol 55, 60-64. Doi: 10.1016/j.biocel.2014.08.011.

Beregi, E., Regius, O., 1987. Comparative morphological study of age related mitochondrial changes of the lymphocytes and skeletal muscle cells. Acta Morphol Hung 35, 219-224. Doi. 
Biala, A.K., Dhingra, R., Kirshenbaum, L.A., 2015. Mitochondrial dynamics: Orchestrating the journey to advanced age. J Mol Cell Cardiol 83, 37-43. Doi: 10.1016/j.yjmcc.2015.04.015.

Biel, T.G., Lee, S., Flores-Toro, J.A., Dean, J.W., Go, K.L., Lee, M.H., Law, B.K., Law, M.E., Dunn, W.A., Jr., Zendejas, I., Behrns, K.E., Kim, J.S., 2016. Sirtuin 1 suppresses mitochondrial dysfunction of ischemic mouse livers in a mitofusin 2-dependent manner. Cell Death Differ 23, 279-290. Doi: 10.1038/cdd.2015.96.

Borengasser, S.J., Faske, J., Kang, P., Blackburn, M.L., Badger, T.M., Shankar, K., 2014. In utero exposure to prepregnancy maternal obesity and postweaning high-fat diet impair regulators of mitochondrial dynamics in rat placenta and offspring. Physiol Genomics 46, 841-850. Doi: 10.1152/physiolgenomics.00059.2014.

Brand, M.D., Nicholls, D.G., 2011. Assessing mitochondrial dysfunction in cells. Biochem J 435, 297-312. Doi: 10.1042/BJ20110162.

Budanov, A.V., Karin, M., 2008. p53 target genes sestrin1 and sestrin2 connect genotoxic stress and mTOR signaling. Cell 134, 451-460. Doi: 10.1016/j.cell.2008.06.028.

Burkewitz, K., Zhang, Y., Mair, W.B., 2014. AMPK at the nexus of energetics and aging. Cell Metab 20, 10-25. Doi: 10.1016/j.cmet.2014.03.002.

Capt, C., Passamonti, M., Breton, S., 2016. The human mitochondrial genome may code for more than 13 proteins. Mitochondrial DNA A DNA Mapp Seq Anal 27, 3098-3101. Doi: 10.3109/19401736.2014.1003924.

Cartoni, R., Leger, B., Hock, M.B., Praz, M., Crettenand, A., Pich, S., Ziltener, J.L., Luthi, F., Deriaz, O., Zorzano, A., Gobelet, C., Kralli, A., Russell, A.P., 
2005. Mitofusins $1 / 2$ and ERRalpha expression are increased in human skeletal muscle after physical exercise. J Physiol 567, 349-358. Doi: 10.1113/jphysiol.2005.092031.

Cartwright, M.J., Tchkonia, T., Kirkland, J.L., 2007. Aging in adipocytes: potential impact of inherent, depot-specific mechanisms. Exp Gerontol 42, 463471. Doi: 10.1016/j.exger.2007.03.003.

Cavalcanti-de-Albuquerque, J.P., Salvador, I.C., Martins, E.L., JardimMesseder, D., Werneck-de-Castro, J.P., Galina, A., Carvalho, D.P., 2014. Role of estrogen on skeletal muscle mitochondrial function in ovariectomized rats: a time course study in different fiber types. J Appl Physiol (1985) 116, 779-789. Doi: 10.1152/japplphysiol.00121.2013.

Civitarese, A.E., Carling, S., Heilbronn, L.K., Hulver, M.H., Ukropcova, B., Deutsch, W.A., Smith, S.R., Ravussin, E., Team, C.P., 2007. Calorie restriction increases muscle mitochondrial biogenesis in healthy humans. PLoS Med 4, e76. Doi: 10.1371/journal.pmed.0040076.

Collins, T.J., Berridge, M.J., Lipp, P., Bootman, M.D., 2002. Mitochondria are morphologically and functionally heterogeneous within cells. EMBO J 21, 16161627. Doi: 10.1093/emboj/21.7.1616.

Conley, K.E., Jubrias, S.A., Esselman, P.C., 2000. Oxidative capacity and ageing in human muscle. J Physiol 526 Pt 1, 203-210. Doi.

Chan, E.Y., 2009. mTORC1 phosphorylates the ULK1-mAtg13-FIP200 autophagy regulatory complex. Sci Signal 2, pe51. Doi: 10.1126/scisignal.284pe51.

Chen, G., Liang, G., Ou, J., Goldstein, J.L., Brown, M.S., 2004. Central role for liver $\mathrm{X}$ receptor in insulin-mediated activation of Srebp-1c transcription and 
stimulation of fatty acid synthesis in liver. Proc Natl Acad Sci U S A 101, 1124511250. Doi: 10.1073/pnas.0404297101.

Chen, H., Chan, D.C., 2005. Emerging functions of mammalian mitochondrial fusion and fission. Hum Mol Genet 14 Spec No. 2, R283-289. Doi: 10.1093/hmg/ddi270.

Chen, H., Chomyn, A., Chan, D.C., 2005. Disruption of fusion results in mitochondrial heterogeneity and dysfunction. J Biol Chem 280, 26185-26192. Doi: $10.1074 / j b c . M 503062200$.

Chistiakov, D.A., Sobenin, I.A., Revin, V.V., Orekhov, A.N., Bobryshev, Y.V., 2014. Mitochondrial aging and age-related dysfunction of mitochondria. Biomed Res Int 2014, 238463. Doi: 10.1155/2014/238463.

Choi, M., Chaudhari, H.N., Ji, Y.R., Ryoo, Z.Y., Kim, S.W., Yun, J.W., 2015. Effect of estrogen on expression of prohibitin in white adipose tissue and liver of diet-induced obese rats. Mol Cell Biochem 407, 181-196. Doi: 10.1007/s11010015-2468-1.

Chung, K.W., Lee, E.K., Kim, D.H., An, H.J., Kim, N.D., Im, D.S., Lee, J., Yu, B.P., Chung, H.Y., 2015. Age-related sensitivity to endotoxin-induced liver inflammation: Implication of inflammasome/IL-1beta for steatohepatitis. Aging Cell 14, 524-533. Doi: 10.1111/acel.12305.

de Brito, O.M., Scorrano, L., 2008. Mitofusin 2 tethers endoplasmic reticulum to mitochondria. Nature 456, 605-610. Doi: 10.1038/nature07534.

de Brito, O.M., Scorrano, L., 2009. Mitofusin-2 regulates mitochondrial and endoplasmic reticulum morphology and tethering: the role of Ras. Mitochondrion 9, 222-226. Doi: 10.1016/j.mito.2009.02.005. 
Dela, F., Helge, J.W., 2013. Insulin resistance and mitochondrial function in skeletal muscle. Int $\mathrm{J}$ Biochem Cell Biol 45, 11-15. Doi: 10.1016/j.biocel.2012.09.019.

Demonacos, C., Djordjevic-Markovic, R., Tsawdaroglou, N., Sekeris, C.E., 1995. The mitochondrion as a primary site of action of glucocorticoids: the interaction of the glucocorticoid receptor with mitochondrial DNA sequences showing partial similarity to the nuclear glucocorticoid responsive elements. J Steroid Biochem Mol Biol 55, 43-55. Doi.

Demonacos, C.V., Karayanni, N., Hatzoglou, E., Tsiriyiotis, C., Spandidos, D.A., Sekeris, C.E., 1996. Mitochondrial genes as sites of primary action of steroid hormones. Steroids 61, 226-232. Doi.

Dietrich, M.O., Liu, Z.W., Horvath, T.L., 2013. Mitochondrial dynamics controlled by mitofusins regulate Agrp neuronal activity and diet-induced obesity. Cell 155, 188-199. Doi: 10.1016/j.cell.2013.09.004.

Dolinsky, V.W., Rogan, K.J., Sung, M.M., Zordoky, B.N., Haykowsky, M.J., Young, M.E., Jones, L.W., Dyck, J.R., 2013. Both aerobic exercise and resveratrol supplementation attenuate doxorubicin-induced cardiac injury in mice. Am J Physiol Endocrinol Metab 305, E243-253. Doi: 10.1152/ajpendo.00044.2013.

Duvel, K., Yecies, J.L., Menon, S., Raman, P., Lipovsky, A.I., Souza, A.L., Triantafellow, E., Ma, Q., Gorski, R., Cleaver, S., Vander Heiden, M.G., MacKeigan, J.P., Finan, P.M., Clish, C.B., Murphy, L.O., Manning, B.D., 2010. Activation of a metabolic gene regulatory network downstream of mTOR complex 1. Mol Cell 39, 171-183. Doi: 10.1016/j.molcel.2010.06.022. 
Fabbri, E., Chia, C.W., Spencer, R.G., Fishbein, K.W., Reiter, D.A., Cameron, D., Zane, A.C., Moore, Z.A., Gonzalez-Freire, M., Zoli, M., Studenski, S.A., Kalyani, R.R., Egan, J.M., Ferrucci, L., 2016. Insulin Resistance is Associated with Reduced Mitochondrial Oxidative Capacity Measured by 31P Magnetic Resonance Spectroscopy in Non-Diabetic Participants from the Baltimore Longitudinal Study of Aging. Diabetes. Doi: 10.2337/db16-0754.

Feige, J.N., Lagouge, M., Auwerx, J., 2008. Dietary manipulation of mouse metabolism. Curr Protoc Mol Biol Chapter 29, Unit 29B 25. Doi: 10.1002/0471142727.mb29b05s84.

Fuku, N., Pareja-Galeano, H., Zempo, H., Alis, R., Arai, Y., Lucia, A., Hirose, N., 2015. The mitochondrial-derived peptide MOTS-c: a player in exceptional longevity? Aging Cell 14, 921-923. Doi: 10.1111/acel.12389.

Gambacciani, M., Ciaponi, M., Cappagli, B., Piaggesi, L., De Simone, L., Orlandi, R., Genazzani, A.R., 1997. Body weight, body fat distribution, and hormonal replacement therapy in early postmenopausal women. J Clin Endocrinol Metab 82, 414-417. Doi: 10.1210/jcem.82.2.3735.

Garcia-Roves, P., Huss, J.M., Han, D.H., Hancock, C.R., Iglesias-Gutierrez, E., Chen, M., Holloszy, J.O., 2007. Raising plasma fatty acid concentration induces increased biogenesis of mitochondria in skeletal muscle. Proc Natl Acad Sci U S A 104, 10709-10713. Doi: 10.1073/pnas.0704024104.

Genova, M.L., Lenaz, G., 2015. The Interplay Between Respiratory Supercomplexes and ROS in Aging. Antioxid Redox Signal 23, 208-238. Doi: 10.1089/ars.2014.6214. 
Gomes, L.C., Scorrano, L., 2008. High levels of Fis1, a pro-fission mitochondrial protein, trigger autophagy. Biochim Biophys Acta 1777, 860-866. Doi: 10.1016/j.bbabio.2008.05.442.

Gomes, L.C., Scorrano, L., 2011. Mitochondrial elongation during autophagy: a stereotypical response to survive in difficult times. Autophagy 7, 1251-1253. Doi: 10.4161/auto.7.10.16771.

Goncalves, R.L., Quinlan, C.L., Perevoshchikova, I.V., Hey-Mogensen, M., Brand, M.D., 2015. Sites of superoxide and hydrogen peroxide production by muscle mitochondria assessed ex vivo under conditions mimicking rest and exercise. J Biol Chem 290, 209-227. Doi: 10.1074/jbc.M114.619072.

Gonzalez-Freire, M., Cabo, R.d., Bernier, M., Sollott, S.J., Fabbri, E., Navas, P., Ferrucci, L., 2015. Reconsidering the Role of Mitochondria in Aging. The Journals of Gerontology Series A: Biological Sciences and Medical Sciences Doi: 10.1093/gerona/glv070. Doi: 10.1093/gerona/glv070.

Goto, T., Takano, M., 2009. Transcriptional role of FOXO1 in drug resistance through antioxidant defense systems. Adv Exp Med Biol 665, 171-179. Doi.

Gouspillou, G., Sgarioto, N., Norris, B., Barbat-Artigas, S., Aubertin-Leheudre, M., Morais, J.A., Burelle, Y., Taivassalo, T., Hepple, R.T., 2014. The relationship between muscle fiber type-specific PGC-1alpha content and mitochondrial content varies between rodent models and humans. PLoS One 9, e103044. Doi: 10.1371/journal.pone.0103044.

Gram, M., Vigelso, A., Yokota, T., Helge, J.W., Dela, F., Hey-Mogensen, M., 2015. Skeletal muscle mitochondrial H2 O2 emission increases with immobilization and decreases after aerobic training in young and older men. $\mathrm{J}$ Physiol 593, 4011-4027. Doi: 10.1113/JP270211. 
Grefhorst, A., Elzinga, B.M., Voshol, P.J., Plosch, T., Kok, T., Bloks, V.W., van der Sluijs, F.H., Havekes, L.M., Romijn, J.A., Verkade, H.J., Kuipers, F., 2002. Stimulation of lipogenesis by pharmacological activation of the liver $\mathrm{X}$ receptor leads to production of large, triglyceride-rich very low density lipoprotein particles. J Biol Chem 277, 34182-34190. Doi: 10.1074/jbc.M204887200.

Griffin, T.M., Humphries, K.M., Kinter, M., Lim, H.Y., Szweda, L.I., 2015. Nutrient sensing and utilization: Getting to the heart of metabolic flexibility. Biochimie. Doi: 10.1016/j.biochi.2015.10.013.

Guarente, L., 2011. Sirtuins, aging, and metabolism. Cold Spring Harb Symp Quant Biol 76, 81-90. Doi: 10.1101/sqb.2011.76.010629.

Guarente, L., 2013. Calorie restriction and sirtuins revisited. Genes Dev 27, 2072-2085. Doi: 10.1101/gad.227439.113.

Gurung, P., Lukens, J.R., Kanneganti, T.D., 2015. Mitochondria: diversity in the regulation of the NLRP3 inflammasome. Trends Mol Med 21, 193-201. Doi: 10.1016/j.molmed.2014.11.008.

Gwinn, D.M., Shackelford, D.B., Egan, D.F., Mihaylova, M.M., Mery, A., Vasquez, D.S., Turk, B.E., Shaw, R.J., 2008. AMPK phosphorylation of raptor mediates a metabolic checkpoint. Mol Cell 30, 214-226. Doi: 10.1016/j.molcel.2008.03.003.

Hancock, C.R., Han, D.H., Chen, M., Terada, S., Yasuda, T., Wright, D.C., Holloszy, J.O., 2008. High-fat diets cause insulin resistance despite an increase in muscle mitochondria. Proc Natl Acad Sci U S A 105, 7815-7820. Doi: 10.1073/pnas.0802057105. 
Handschin, C., Spiegelman, B.M., 2006. Peroxisome proliferator-activated receptor gamma coactivator 1 coactivators, energy homeostasis, and metabolism. Endocr Rev 27, 728-735. Doi: 10.1210/er.2006-0037.

Hardie, D.G., 2011. Sensing of energy and nutrients by AMP-activated protein kinase. Am J Clin Nutr 93, 891S-896. Doi: 10.3945/ajcn.110.001925.

Hardie, D.G., Ross, F.A., Hawley, S.A., 2012. AMPK: a nutrient and energy sensor that maintains energy homeostasis. Nat Rev Mol Cell Biol 13, 251-262. Doi: $10.1038 / \mathrm{nrm} 3311$.

Harijith, A., Ebenezer, D.L., Natarajan, V., 2014. Reactive oxygen species at the crossroads of inflammasome and inflammation. Front Physiol 5, 352. Doi: 10.3389/fphys.2014.00352.

He, B., Kim, T.H., Kommagani, R., Feng, Q., Lanz, R.B., Jeong, J.W., DeMayo, F.J., Katzenellenbogen, B.S., Lydon, J.P., O'Malley, B.W., 2011. Estrogenregulated prohibitin is required for mouse uterine development and adult function. Endocrinology 152, 1047-1056. Doi: 10.1210/en.2010-0732.

Holmstrom, M.H., Iglesias-Gutierrez, E., Zierath, J.R., Garcia-Roves, P.M., 2012. Tissue-specific control of mitochondrial respiration in obesity-related insulin resistance and diabetes. Am J Physiol Endocrinol Metab 302, E731-739. Doi: 10.1152/ajpendo.00159.2011.

Hou, Y.S., Guan, J.J., Xu, H.D., Wu, F., Sheng, R., Qin, Z.H., 2015. Sestrin2 Protects Dopaminergic Cells against Rotenone Toxicity through AMPKDependent Autophagy Activation. Mol Cell Biol 35, 2740-2751. Doi: 10.1128/MCB.00285-15.

Huffman, D.M., Barzilai, N., 2009. Role of visceral adipose tissue in aging. Biochim Biophys Acta 1790, 1117-1123. Doi: 10.1016/j.bbagen.2009.01.008. 
Imai, S., Guarente, L., 2010. Ten years of NAD-dependent SIR2 family deacetylases: implications for metabolic diseases. Trends Pharmacol Sci 31, 212-220. Doi: 10.1016/j.tips.2010.02.003.

Inoki, K., Zhu, T., Guan, K.L., 2003. TSC2 mediates cellular energy response to control cell growth and survival. Cell 115, 577-590. Doi.

Ishihara, M., Urushido, M., Hamada, K., Matsumoto, T., Shimamura, Y., Ogata, K., Inoue, K., Taniguchi, Y., Horino, T., Fujieda, M., Fujimoto, S., Terada, Y., 2013. Sestrin-2 and BNIP3 regulate autophagy and mitophagy in renal tubular cells in acute kidney injury. Am J Physiol Renal Physiol 305, F495-509. Doi: 10.1152/ajprenal.00642.2012.

Jager, S., Handschin, C., St-Pierre, J., Spiegelman, B.M., 2007. AMP-activated protein kinase (AMPK) action in skeletal muscle via direct phosphorylation of PGC-1alpha. Proc Natl Acad Sci U S A 104, 12017-12022. Doi: 10.1073/pnas.0705070104.

Jheng, H.F., Huang, S.H., Kuo, H.M., Hughes, M.W., Tsai, Y.S., 2015. Molecular insight and pharmacological approaches targeting mitochondrial dynamics in skeletal muscle during obesity. Ann N Y Acad Sci 1350, 82-94. Doi: 10.1111/nyas. 12863 .

Jheng, H.F., Tsai, P.J., Guo, S.M., Kuo, L.H., Chang, C.S., Su, I.J., Chang, C.R., Tsai, Y.S., 2012. Mitochondrial fission contributes to mitochondrial dysfunction and insulin resistance in skeletal muscle. Mol Cell Biol 32, 309-319. Doi: 10.1128/MCB.05603-11.

Jin, J., Gu, H., Anders, N.M., Ren, T., Jiang, M., Tao, M., Peng, Q., Rudek, M.A., Duan, W., 2016. Metformin Protects Cells from Mutant Huntingtin Toxicity 
Through Activation of AMPK and Modulation of Mitochondrial Dynamics. Neuromolecular Med. Doi: 10.1007/s12017-016-8412-z.

Jin, S.H., Yang, J.H., Shin, B.Y., Seo, K., Shin, S.M., Cho, I.J., Ki, S.H., 2013. Resveratrol inhibits LXRalpha-dependent hepatic lipogenesis through novel antioxidant Sestrin2 gene induction. Toxicol Appl Pharmacol 271, 95-105. Doi: 10.1016/j.taap.2013.04.023.

Joseph, S.B., Laffitte, B.A., Patel, P.H., Watson, M.A., Matsukuma, K.E., Walczak, R., Collins, J.L., Osborne, T.F., Tontonoz, P., 2002. Direct and indirect mechanisms for regulation of fatty acid synthase gene expression by liver $\mathrm{X}$ receptors. J Biol Chem 277, 11019-11025. Doi: 10.1074/jbc.M111041200.

Kammeyer, A., Luiten, R.M., 2015. Oxidation events and skin aging. Ageing Res Rev 21, 16-29. Doi: 10.1016/j.arr.2015.01.001.

Kang, T., Lu, W., Xu, W., Anderson, L., Bacanamwo, M., Thompson, W., Chen, Y.E., Liu, D., 2013. MicroRNA-27 (miR-27) targets prohibitin and impairs adipocyte differentiation and mitochondrial function in human adipose-derived stem cells. J Biol Chem 288, 34394-34402. Doi: 10.1074/jbc.M113.514372.

Karakelides, H., Irving, B.A., Short, K.R., O'Brien, P., Nair, K.S., 2010. Age, obesity, and sex effects on insulin sensitivity and skeletal muscle mitochondrial function. Diabetes 59, 89-97. Doi: 10.2337/db09-0591.

Kendrick, A.A., Choudhury, M., Rahman, S.M., McCurdy, C.E., Friederich, M., Van Hove, J.L., Watson, P.A., Birdsey, N., Bao, J., Gius, D., Sack, M.N., Jing, E., Kahn, C.R., Friedman, J.E., Jonscher, K.R., 2011. Fatty liver is associated with reduced SIRT3 activity and mitochondrial protein hyperacetylation. Biochem J 433, 505-514. Doi: 10.1042/BJ20100791. 
Kennedy, B.K., Berger, S.L., Brunet, A., Campisi, J., Cuervo, A.M., Epel, E.S., Franceschi, C., Lithgow, G.J., Morimoto, R.I., Pessin, J.E., Rando, T.A., Richardson, A., Schadt, E.E., Wyss-Coray, T., Sierra, F., 2014. Geroscience: linking aging to chronic disease. Cell 159, 709-713. Doi: 10.1016/j.cell.2014.10.039.

Kim, J., Kundu, M., Viollet, B., Guan, K.L., 2011. AMPK and mTOR regulate autophagy through direct phosphorylation of Ulk1. Nat Cell Biol 13, 132-141. Doi: $10.1038 / \mathrm{ncb} 2152$.

Kirkland, J.L., Tchkonia, T., Pirtskhalava, T., Han, J., Karagiannides, I., 2002. Adipogenesis and aging: does aging make fat go MAD? Exp Gerontol 37, 757767. Doi.

Koliaki, C., Roden, M., 2016. Alterations of Mitochondrial Function and Insulin Sensitivity in Human Obesity and Diabetes Mellitus. Annu Rev Nutr. Doi: 10.1146/annurev-nutr-071715-050656.

Kovalenko, E.I., Boyko, A.A., Semenkov, V.F., Lutsenko, G.V., Grechikhina, M.V., Kanevskiy, L.M., Azhikina, T.L., Telford, W.G., Sapozhnikov, A.M., 2014. ROS production, intracellular HSP70 levels and their relationship in human neutrophils: effects of age. Oncotarget 5, 11800-11812. Doi: 10.18632/oncotarget.2856.

Kuk, J.L., Saunders, T.J., Davidson, L.E., Ross, R., 2009. Age-related changes in total and regional fat distribution. Ageing Res Rev 8, 339-348. Doi: 10.1016/j.arr.2009.06.001.

Kume, S., Uzu, T., Horiike, K., Chin-Kanasaki, M., Isshiki, K., Araki, S., Sugimoto, T., Haneda, M., Kashiwagi, A., Koya, D., 2010. Calorie restriction enhances cell adaptation to hypoxia through Sirt1-dependent mitochondrial 
autophagy in mouse aged kidney. J Clin Invest 120, 1043-1055. Doi: 10.1172/JCI41376.

Lane, R.K., Hilsabeck, T., Rea, S.L., 2015. The role of mitochondrial dysfunction in age-related diseases. Biochim Biophys Acta 1847, 1387-1400. Doi: 10.1016/j.bbabio.2015.05.021.

Laplante, M., Sabatini, D.M., 2009. mTOR signaling at a glance. J Cell Sci 122, 3589-3594. Doi: 10.1242/jcs.051011.

Larsen, S., Hey-Mogensen, M., Rabol, R., Stride, N., Helge, J.W., Dela, F., 2012. The influence of age and aerobic fitness: effects on mitochondrial respiration in skeletal muscle. Acta Physiol (Oxf) 205, 423-432. Doi: 10.1111/j.1748-1716.2012.02408.x.

Larson-Meyer, D.E., Heilbronn, L.K., Redman, L.M., Newcomer, B.R., Frisard, M.I., Anton, S., Smith, S.R., Alfonso, A., Ravussin, E., 2006. Effect of calorie restriction with or without exercise on insulin sensitivity, beta-cell function, fat cell size, and ectopic lipid in overweight subjects. Diabetes Care 29, 1337-1344. Doi: $10.2337 / \mathrm{dc} 05-2565$.

Lee, C., Kim, K.H., Cohen, P., 2016. MOTS-c: A novel mitochondrial-derived peptide regulating muscle and fat metabolism. Free Radic Biol Med. Doi: 10.1016/j.freeradbiomed.2016.05.015.

Lee, C., Yen, K., Cohen, P., 2013a. Humanin: a harbinger of mitochondrialderived peptides? Trends Endocrinol Metab 24, 222-228. Doi: 10.1016/j.tem.2013.01.005.

Lee, C., Zeng, J., Drew, B.G., Sallam, T., Martin-Montalvo, A., Wan, J., Kim, S.J., Mehta, H., Hevener, A.L., de Cabo, R., Cohen, P., 2015. The mitochondrial-derived peptide MOTS-c promotes metabolic homeostasis and 
reduces obesity and insulin resistance. Cell Metab 21, 443-454. Doi: 10.1016/j.cmet.2015.02.009.

Lee, J.H., Budanov, A.V., Karin, M., 2013b. Sestrins orchestrate cellular metabolism to attenuate aging. Cell Metab 18, 792-801. Doi: 10.1016/j.cmet.2013.08.018.

Lee, J.H., Budanov, A.V., Park, E.J., Birse, R., Kim, T.E., Perkins, G.A., Ocorr, K., Ellisman, M.H., Bodmer, R., Bier, E., Karin, M., 2010. Sestrin as a feedback inhibitor of TOR that prevents age-related pathologies. Science 327, 12231228. Doi: $10.1126 /$ science. 1182228.

Lee, J.H., Budanov, A.V., Talukdar, S., Park, E.J., Park, H.L., Park, H.W., Bandyopadhyay, G., Li, N., Aghajan, M., Jang, I., Wolfe, A.M., Perkins, G.A., Ellisman, M.H., Bier, E., Scadeng, M., Foretz, M., Viollet, B., Olefsky, J., Karin, M., 2012. Maintenance of metabolic homeostasis by Sestrin2 and Sestrin3. Cell Metab 16, 311-321. Doi: 10.1016/j.cmet.2012.08.004.

Lee, S., Jeong, S.Y., Lim, W.C., Kim, S., Park, Y.Y., Sun, X., Youle, R.J., Cho, H., 2007. Mitochondrial fission and fusion mediators, hFis1 and OPA1, modulate cellular senescence. J Biol Chem 282, 22977-22983. Doi: 10.1074/jbc.M700679200.

Lee, T.H., Pastorino, L., Lu, K.P., 2011. Peptidyl-prolyl cis-trans isomerase Pin1 in ageing, cancer and Alzheimer disease. Expert Rev Mol Med 13, e21. Doi: 10.1017/S1462399411001906.

Li, G.H., Lin, X.L., Zhang, H., Li, S., He, X.L., Zhang, K., Peng, J., Tang, Y.L., Zeng, J.F., Zhao, Y., Ma, X.F., Lei, J.J., Wang, R., Wei, D.H., Jiang, Z.S., Wang, Z., 2015. Ox-Lp(a) transiently induces HUVEC autophagy via an ROS- 
dependent PAPR-1-LKB1-AMPK-mTOR pathway. Atherosclerosis 243, 223235. Doi: 10.1016/j.atherosclerosis.2015.09.020.

Li, Y., Xu, S., Mihaylova, M.M., Zheng, B., Hou, X., Jiang, B., Park, O., Luo, Z., Lefai, E., Shyy, J.Y., Gao, B., Wierzbicki, M., Verbeuren, T.J., Shaw, R.J., Cohen, R.A., Zang, M., 2011. AMPK phosphorylates and inhibits SREBP activity to attenuate hepatic steatosis and atherosclerosis in diet-induced insulin-resistant mice. Cell Metab 13, 376-388. Doi: 10.1016/j.cmet.2011.03.009.

Liesa, M., Borda-d'Agua, B., Medina-Gomez, G., Lelliott, C.J., Paz, J.C., Rojo, M., Palacin, M., Vidal-Puig, A., Zorzano, A., 2008. Mitochondrial fusion is increased by the nuclear coactivator PGC-1beta. PLoS One 3, e3613. Doi: 10.1371/journal.pone.0003613.

Liesa, M., Palacin, M., Zorzano, A., 2009. Mitochondrial dynamics in mammalian health and disease. Physiol Rev 89, 799-845. Doi: 10.1152/physrev.00030.2008.

Liu, X.M., Chan, H.C., Ding, G.L., Cai, J., Song, Y., Wang, T.T., Zhang, D., Chen, H., Yu, M.K., Wu, Y.T., Qu, F., Liu, Y., Lu, Y.C., Adashi, E.Y., Sheng, J.Z., Huang, H.F., 2015. FSH regulates fat accumulation and redistribution in aging through the Galphai/Ca(2+)/CREB pathway. Aging Cell 14, 409-420. Doi: 10.1111/acel.12331.

Liu, Y., Diaz, V., Fernandez, E., Strong, R., Ye, L., Baur, J.A., Lamming, D.W., Richardson, A., Salmon, A.B., 2014. Rapamycin-induced metabolic defects are reversible in both lean and obese mice. Aging (Albany NY) 6, 742-754. Doi: 10.18632/aging. 100688. 
Lokireddy, S., Wijesoma, I.W., Teng, S., Bonala, S., Gluckman, P.D., McFarlane, C., Sharma, M., Kambadur, R., 2012. The ubiquitin ligase Mul1 induces mitophagy in skeletal muscle in response to muscle-wasting stimuli. Cell Metab 16, 613-624. Doi: 10.1016/j.cmet.2012.10.005.

Lopez-Lluch, G., Irusta, P.M., Navas, P., de Cabo, R., 2008. Mitochondrial biogenesis and healthy aging. Exp Gerontol 43, 813-819. Doi: 10.1016/j.exger.2008.06.014.

Lopez-Lluch, G., Navas, P., 2015. Calorie restriction as an intervention in ageing. J Physiol. Doi: 10.1113/JP270543.

Lopez-Lluch, G., Navas, P., 2016. Calorie restriction as an intervention in ageing. J Physiol 594, 2043-2060. Doi: 10.1113/JP270543.

Lopez-Otin, C., Blasco, M.A., Partridge, L., Serrano, M., Kroemer, G., 2013. The hallmarks of aging. Cell 153, 1194-1217. Doi: 10.1016/j.cell.2013.05.039. Loson, O.C., Song, Z., Chen, H., Chan, D.C., 2013. Fis1, Mff, MiD49, and MiD51 mediate Drp1 recruitment in mitochondrial fission. Mol Biol Cell 24, 659667. Doi: 10.1091/mbc.E12-10-0721.

Lourenco, A.B., Munoz-Jimenez, C., Venegas-Caleron, M., Artal-Sanz, M., 2015. Analysis of the effect of the mitochondrial prohibitin complex, a contextdependent modulator of longevity, on the C. elegans metabolome. Biochim Biophys Acta 1847, 1457-1468. Doi: 10.1016/j.bbabio.2015.06.003.

Lowell, B.B., Shulman, G.I., 2005. Mitochondrial dysfunction and type 2 diabetes. Science 307, 384-387. Doi: 10.1126/science.1104343.

Lu, T., Finkel, T., 2008. Free radicals and senescence. Exp Cell Res 314, 19181922. Doi: 10.1016/j.yexcr.2008.01.011. 
Lupfer, C.R., Kanneganti, T.D., 2012. The role of inflammasome modulation in virulence. Virulence 3, 262-270. Doi: 10.4161/viru.20266.

Mahboobi, H., Golmirzaei, J., Gan, S.H., Jalalian, M., Kamal, M.A., 2014. Humanin: a possible linkage between Alzheimer's disease and type 2 diabetes. CNS Neurol Disord Drug Targets 13, 543-552. Doi.

Mai, S., Klinkenberg, M., Auburger, G., Bereiter-Hahn, J., Jendrach, M., 2010. Decreased expression of Drp1 and Fis1 mediates mitochondrial elongation in senescent cells and enhances resistance to oxidative stress through PINK1. J Cell Sci 123, 917-926. Doi: 10.1242/jcs.059246.

Martin-Montalvo, A., de Cabo, R., 2013. Mitochondrial metabolic reprogramming induced by calorie restriction. Antioxid Redox Signal 19, 310320. Doi: $10.1089 /$ ars.2012.4866.

Matsumoto, A.M., 2002. Andropause: clinical implications of the decline in serum testosterone levels with aging in men. J Gerontol A Biol Sci Med Sci 57, M76-99. Doi.

Mattagajasingh, I., Kim, C.S., Naqvi, A., Yamamori, T., Hoffman, T.A., Jung, S.B., DeRicco, J., Kasuno, K., Irani, K., 2007. SIRT1 promotes endotheliumdependent vascular relaxation by activating endothelial nitric oxide synthase. Proc Natl Acad Sci U S A 104, 14855-14860. Doi: 10.1073/pnas.0704329104.

McDonagh, B., 2016. Editorial: Redox Regulation in Skeletal Muscle Aging and Exercise. Front Physiol 7, 5. Doi: 10.3389/fphys.2016.00005.

McRae, S., Iqbal, J., Sarkar-Dutta, M., Lane, S., Nagaraj, A., Ali, N., Waris, G., 2015. Hepatitis C virus-induced NLRP3-Inflammasome Activates the Sterol Regulatory Element Binding Protein (SREBP) and Regulates Lipid Metabolism. J Biol Chem. Doi: 10.1074/jbc.M115.694059. 
Mercken, E.M., Carboneau, B.A., Krzysik-Walker, S.M., de Cabo, R., 2012. Of mice and men: the benefits of caloric restriction, exercise, and mimetics. Ageing Res Rev 11, 390-398. Doi: 10.1016/j.arr.2011.11.005.

Merkwirth, C., Martinelli, P., Korwitz, A., Morbin, M., Bronneke, H.S., Jordan, S.D., Rugarli, E.I., Langer, T., 2012. Loss of prohibitin membrane scaffolds impairs mitochondrial architecture and leads to tau hyperphosphorylation and neurodegeneration. PLoS Genet 8, e1003021. Doi: 10.1371/journal.pgen.1003021.

Merry, B.J., 2002. Molecular mechanisms linking calorie restriction and longevity. Int J Biochem Cell Biol 34, 1340-1354. Doi.

Mootha, V.K., Lindgren, C.M., Eriksson, K.F., Subramanian, A., Sihag, S., Lehar, J., Puigserver, P., Carlsson, E., Ridderstrale, M., Laurila, E., Houstis, N., Daly, M.J., Patterson, N., Mesirov, J.P., Golub, T.R., Tamayo, P., Spiegelman, B., Lander, E.S., Hirschhorn, J.N., Altshuler, D., Groop, L.C., 2003. PGC1alpha-responsive genes involved in oxidative phosphorylation are coordinately downregulated in human diabetes. Nat Genet 34, 267-273. Doi: 10.1038/ng1180.

Mudali, S., Dobs, A.S., 2004. Effects of testosterone on body composition of the aging male. Mech Ageing Dev 125, 297-304. Doi: 10.1016/j.mad.2004.01.004. Nakatsu, Y., Iwashita, M., Sakoda, H., Ono, H., Nagata, K., Matsunaga, Y., Fukushima, T., Fujishiro, M., Kushiyama, A., Kamata, H., Takahashi, S., Katagiri, H., Honda, H., Kiyonari, H., Uchida, T., Asano, T., 2015. Prolyl isomerase Pin1 negatively regulates AMP-activated protein kinase (AMPK) by associating with the CBS domain in the gamma subunit. J Biol Chem 290, 24255-24266. Doi: 10.1074/jbc.M115.658559. 
Nakatsu, Y., Sakoda, H., Kushiyama, A., Zhang, J., Ono, H., Fujishiro, M., Kikuchi, T., Fukushima, T., Yoneda, M., Ohno, H., Horike, N., Kanna, M., Tsuchiya, Y., Kamata, H., Nishimura, F., Isobe, T., Ogihara, T., Katagiri, H., Oka, Y., Takahashi, S., Kurihara, H., Uchida, T., Asano, T., 2011. Peptidylprolyl cis/trans isomerase NIMA-interacting 1 associates with insulin receptor substrate-1 and enhances insulin actions and adipogenesis. J Biol Chem 286, 20812-20822. Doi: 10.1074/jbc.M110.206904.

Nasrallah, C.M., Horvath, T.L., 2014. Mitochondrial dynamics in the central regulation of metabolism. Nat Rev Endocrinol 10, 650-658. Doi: 10.1038/nrendo.2014.160.

Nemoto, S., Fergusson, M.M., Finkel, T., 2005. SIRT1 functionally interacts with the metabolic regulator and transcriptional coactivator PGC-1\{alpha\}. J Biol Chem 280, 16456-16460. Doi: 10.1074/jbc.M501485200.

Newsholme, P., de Bittencourt, P.I., Jr., 2014. The fat cell senescence hypothesis: a mechanism responsible for abrogating the resolution of inflammation in chronic disease. Curr Opin Clin Nutr Metab Care 17, 295-305. Doi: 10.1097/MCO.0000000000000077.

Nisoli, E., Tonello, C., Cardile, A., Cozzi, V., Bracale, R., Tedesco, L., Falcone, S., Valerio, A., Cantoni, O., Clementi, E., Moncada, S., Carruba, M.O., 2005. Calorie restriction promotes mitochondrial biogenesis by inducing the expression of eNOS. Science 310, 314-317. Doi: 10.1126/science.1117728.

Palacios, O.M., Carmona, J.J., Michan, S., Chen, K.Y., Manabe, Y., Ward, J.L., 3rd, Goodyear, L.J., Tong, Q., 2009. Diet and exercise signals regulate SIRT3 and activate AMPK and PGC-1alpha in skeletal muscle. Aging (Albany NY) 1, 771-783. Doi. 
Palmer, B.F., Clegg, D.J., 2015. The sexual dimorphism of obesity. Mol Cell Endocrinol 402, 113-119. Doi: 10.1016/j.mce.2014.11.029.

Palus, S., von Haehling, S., Springer, J., 2014. Muscle wasting: an overview of recent developments in basic research. J Cachexia Sarcopenia Muscle 5, 193198. Doi: 10.1007/s13539-014-0157-7.

Park, Y.Y., Lee, S., Karbowski, M., Neutzner, A., Youle, R.J., Cho, H., 2010. Loss of MARCH5 mitochondrial E3 ubiquitin ligase induces cellular senescence through dynamin-related protein 1 and mitofusin 1 . J Cell Sci 123, 619-626. Doi: $10.1242 / \mathrm{jcs} .061481$.

Patti, M.E., Corvera, S., 2010. The role of mitochondria in the pathogenesis of type 2 diabetes. Endocr Rev 31, 364-395. Doi: 10.1210/er.2009-0027.

Payne, B.A., Chinnery, P.F., 2015. Mitochondrial dysfunction in aging: Much progress but many unresolved questions. Biochim Biophys Acta 1847, 13471353. Doi: 10.1016/j.bbabio.2015.05.022.

Peng, J., Ren, K.D., Yang, J., Luo, X.J., 2016. Mitochondrial E3 ubiquitin ligase 1: A key enzyme in regulation of mitochondrial dynamics and functions. Mitochondrion 28, 49-53. Doi: 10.1016/j.mito.2016.03.007.

Pich, S., Bach, D., Briones, P., Liesa, M., Camps, M., Testar, X., Palacin, M., Zorzano, A., 2005. The Charcot-Marie-Tooth type 2A gene product, Mfn2, upregulates fuel oxidation through expression of OXPHOS system. Hum Mol Genet 14, 1405-1415. Doi: 10.1093/hmg/ddi149.

Psarra, A.M., Solakidi, S., Sekeris, C.E., 2006. The mitochondrion as a primary site of action of regulatory agents involved in neuroimmunomodulation. Ann N Y Acad Sci 1088, 12-22. Doi: 10.1196/annals.1366.019. 
Putti, R., Sica, R., Migliaccio, V., Lionetti, L., 2015. Diet impact on mitochondrial bioenergetics and dynamics. Front Physiol 6, 109. Doi: 10.3389/fphys.2015.00109.

Qiu, X., Brown, K., Hirschey, M.D., Verdin, E., Chen, D., 2010. Calorie restriction reduces oxidative stress by SIRT3-mediated SOD2 activation. Cell Metab 12, 662-667. Doi: 10.1016/j.cmet.2010.11.015.

Quiros, P.M., Mottis, A., Auwerx, J., 2016. Mitonuclear communication in homeostasis and stress. Nat Rev Mol Cell Biol 17, 213-226. Doi: 10.1038/nrm.2016.23.

Rattan, S.I., 2008. Hormesis in aging. Ageing Res Rev 7, 63-78. Doi: 10.1016/j.arr.2007.03.002.

Repa, J.J., Liang, G., Ou, J., Bashmakov, Y., Lobaccaro, J.M., Shimomura, I., Shan, B., Brown, M.S., Goldstein, J.L., Mangelsdorf, D.J., 2000. Regulation of mouse sterol regulatory element-binding protein-1c gene (SREBP-1c) by oxysterol receptors, LXRalpha and LXRbeta. Genes Dev 14, 2819-2830. Doi.

Rhee, S.G., Bae, S.H., 2015. The antioxidant function of sestrins is mediated by promotion of autophagic degradation of Keap1 and Nrf2 activation and by inhibition of mTORC1. Free Radic Biol Med 88, 205-211. Doi: 10.1016/j.freeradbiomed.2015.06.007.

Riera, C.E., Merkwirth, C., De Magalhaes Filho, C.D., Dillin, A., 2016. Signaling Networks Determining Life Span. Annu Rev Biochem 85, 35-64. Doi: 10.1146/annurev-biochem-060815-014451.

Ristow, M., 2014. Unraveling the truth about antioxidants: mitohormesis explains ROS-induced health benefits. Nat Med 20, 709-711. Doi: 10.1038/nm.3624. 
Rodriguez-Bies, E., Navas, P., Lopez-Lluch, G., 2015. Age-dependent effect of every-other-day feeding and aerobic exercise in ubiquinone levels and related antioxidant activities in mice muscle. J Gerontol A Biol Sci Med Sci 70, 33-43. Doi: $10.1093 /$ gerona/glu002.

Rodriguez-Bies, E., Tung, B.T., Navas, P., Lopez-Lluch, G., 2016. Resveratrol primes the effects of physical activity in old mice. Br J Nutr 116, 979-988. Doi: 10.1017/S0007114516002920.

Ryan, B.J., Hoek, S., Fon, E.A., Wade-Martins, R., 2015. Mitochondrial dysfunction and mitophagy in Parkinson's: from familial to sporadic disease. Trends Biochem Sci 40, 200-210. Doi: 10.1016/j.tibs.2015.02.003.

Samant, S.A., Zhang, H.J., Hong, Z., Pillai, V.B., Sundaresan, N.R., Wolfgeher, D., Archer, S.L., Chan, D.C., Gupta, M.P., 2014. SIRT3 deacetylates and activates OPA1 to regulate mitochondrial dynamics during stress. Mol Cell Biol 34, 807-819. Doi: 10.1128/MCB.01483-13.

Sanchis-Gomar, F., 2013. Sestrins: novel antioxidant and AMPK-modulating functions regulated by exercise? J Cell Physiol 228, 1647-1650. Doi: 10.1002/jcp.24338.

Santa-Cruz Calvo, S., Navas, P., López-Lluch, G., 2012. Sirtuin-dependent meabolic control and its role in the aging process, In: Clark, K., B. (Ed.), Bioenergetics. InTech, Rijeka, Croatia, pp. 95-120.

Santanasto, A.J., Goodpaster, B.H., Kritchevsky, S.B., Miljkovic, I., Satterfield, S., Schwartz, A.V., Cummings, S.R., Boudreau, R.M., Harris, T.B., Newman, A.B., 2016. Body Composition Remodeling and Mortality: The Health Aging and Body Composition Study. J Gerontol A Biol Sci Med Sci. Doi: 10.1093/gerona/glw163. 
Santel, A., Frank, S., 2008. Shaping mitochondria: The complex posttranslational regulation of the mitochondrial fission protein DRP1. IUBMB Life 60, 448-455. Doi: 10.1002/iub.71.

Sastre-Serra, J., Nadal-Serrano, M., Pons, D.G., Roca, P., Oliver, J., 2012. Mitochondrial dynamics is affected by 17 beta-estradiol in the MCF-7 breast cancer cell line. Effects on fusion and fission related genes. Int J Biochem Cell Biol 44, 1901-1905. Doi: 10.1016/j.biocel.2012.07.012.

Sastre-Serra, J., Nadal-Serrano, M., Pons, D.G., Roca, P., Oliver, J., 2013. The over-expression of ERbeta modifies estradiol effects on mitochondrial dynamics in breast cancer cell line. Int J Biochem Cell Biol 45, 1509-1515. Doi: 10.1016/j.biocel.2013.04.007.

Sataranatarajan, K., Ikeno, Y., Bokov, A., Feliers, D., Yalamanchili, H., Lee, H.J., Mariappan, M.M., Tabatabai-Mir, H., Diaz, V., Prasad, S., Javors, M.A., Ghosh Choudhury, G., Hubbard, G.B., Barnes, J.L., Richardson, A., Kasinath, B.S., 2016. Rapamycin Increases Mortality in $\mathrm{db} / \mathrm{db}$ Mice, a Mouse Model of Type 2 Diabetes. J Gerontol A Biol Sci Med Sci 71, 850-857. Doi: 10.1093/gerona/glv170.

Scarpace, P.J., Matheny, M., Strehler, K.Y., Toklu, H.Z., Kirichenko, N., Carter, C.S., Morgan, D., Tumer, N., 2016. Rapamycin Normalizes Serum Leptin by Alleviating Obesity and Reducing Leptin Synthesis in Aged Rats. J Gerontol A Biol Sci Med Sci 71, 891-899. Doi: 10.1093/gerona/glu230.

Scialo, F., Sriram, A., Fernandez-Ayala, D., Gubina, N., Lohmus, M., Nelson, G., Logan, A., Cooper, H.M., Navas, P., Enriquez, J.A., Murphy, M.P., Sanz, A., 2016. Mitochondrial ROS Produced via Reverse Electron Transport Extend Animal Lifespan. Cell Metab 23, 725-734. Doi: 10.1016/j.cmet.2016.03.009. 
Scheller, K., Seibel, P., Sekeris, C.E., 2003. Glucocorticoid and thyroid hormone receptors in mitochondria of animal cells. Int Rev Cytol 222, 1-61. Doi. Schneeberger, M., Dietrich, M.O., Sebastian, D., Imbernon, M., Castano, C., Garcia, A., Esteban, Y., Gonzalez-Franquesa, A., Rodriguez, I.C., Bortolozzi, A., Garcia-Roves, P.M., Gomis, R., Nogueiras, R., Horvath, T.L., Zorzano, A., Claret, M., 2013. Mitofusin 2 in POMC neurons connects ER stress with leptin resistance and energy imbalance. Cell 155, 172-187. Doi: 10.1016/j.cell.2013.09.003.

Sekeris, C.E., 1990. The mitochondrial genome: a possible primary site of action of steroid hormones. In Vivo 4, 317-320. Doi.

Senesi, P., Montesano, A., Luzi, L., Codella, R., Benedini, S., Terruzzi, I., 2016. Metformin Treatment Prevents Sedentariness Related Damages in Mice. J Diabetes Res 2016, 8274689. Doi: 10.1155/2016/8274689.

Shen, Q., Yamano, K., Head, B.P., Kawajiri, S., Cheung, J.T., Wang, C., Cho, J.H., Hattori, N., Youle, R.J., van der Bliek, A.M., 2014. Mutations in Fis1 disrupt orderly disposal of defective mitochondria. Mol Biol Cell 25, 145-159. Doi: 10.1091/mbc.E13-09-0525.

Short, K.R., Bigelow, M.L., Kahl, J., Singh, R., Coenen-Schimke, J., Raghavakaimal, S., Nair, K.S., 2005. Decline in skeletal muscle mitochondrial function with aging in humans. Proc Natl Acad Sci U S A 102, 5618-5623. Doi: 10.1073/pnas.0501559102.

Simoneau, J.A., Veerkamp, J.H., Turcotte, L.P., Kelley, D.E., 1999. Markers of capacity to utilize fatty acids in human skeletal muscle: relation to insulin resistance and obesity and effects of weight loss. FASEB J 13, 2051-2060. Doi. 
Smirnova, E., Griparic, L., Shurland, D.L., van der Bliek, A.M., 2001. Dynaminrelated protein Drp1 is required for mitochondrial division in mammalian cells. Mol Biol Cell 12, 2245-2256. Doi.

Soriano, F.X., Liesa, M., Bach, D., Chan, D.C., Palacin, M., Zorzano, A., 2006. Evidence for a mitochondrial regulatory pathway defined by peroxisome proliferator-activated receptor-gamma coactivator-1 alpha, estrogen-related receptor-alpha, and mitofusin 2. Diabetes 55, 1783-1791. Doi: 10.2337/db050509.

Sreekumar, P.G., Ishikawa, K., Spee, C., Mehta, H.H., Wan, J., Yen, K., Cohen, P., Kannan, R., Hinton, D.R., 2016. The Mitochondrial-Derived Peptide Humanin Protects RPE Cells From Oxidative Stress, Senescence, and Mitochondrial Dysfunction. Invest Ophthalmol Vis Sci 57, 1238-1253. Doi: 10.1167/iovs.15-17053.

Steinberg, G.R., O'Neill, H.M., Dzamko, N.L., Galic, S., Naim, T., Koopman, R., Jorgensen, S.B., Honeyman, J., Hewitt, K., Chen, Z.P., Schertzer, J.D., Scott, J.W., Koentgen, F., Lynch, G.S., Watt, M.J., van Denderen, B.J., Campbell, D.J., Kemp, B.E., 2010. Whole body deletion of AMP-activated protein kinase \{beta\}2 reduces muscle AMPK activity and exercise capacity. J Biol Chem 285, 37198-37209. Doi: 10.1074/jbc.M110.102434.

Stienstra, R., van Diepen, J.A., Tack, C.J., Zaki, M.H., van de Veerdonk, F.L., Perera, D., Neale, G.A., Hooiveld, G.J., Hijmans, A., Vroegrijk, I., van den Berg, S., Romijn, J., Rensen, P.C., Joosten, L.A., Netea, M.G., Kanneganti, T.D., 2011. Inflammasome is a central player in the induction of obesity and insulin resistance. Proc Natl Acad Sci U S A 108, 15324-15329. Doi: 10.1073/pnas. 1100255108. 
Storlien, L., Oakes, N.D., Kelley, D.E., 2004. Metabolic flexibility. Proc Nutr Soc 63, 363-368. Doi: 10.1079/PNS2004349.

Supale, S., Thorel, F., Merkwirth, C., Gjinovci, A., Herrera, P.L., Scorrano, L., Meda, P., Langer, T., Maechler, P., 2013. Loss of prohibitin induces mitochondrial damages altering beta-cell function and survival and is responsible for gradual diabetes development. Diabetes 62, 3488-3499. Doi: $10.2337 / \mathrm{db} 13-0152$.

Tao, R., Xiong, X., Liangpunsakul, S., Dong, X.C., 2015. Sestrin 3 protein enhances hepatic insulin sensitivity by direct activation of the mTORC2-Akt signaling. Diabetes 64, 1211-1223. Doi: 10.2337/db14-0539.

Theiss, A.L., Vijay-Kumar, M., Obertone, T.S., Jones, D.P., Hansen, J.M., Gewirtz, A.T., Merlin, D., Sitaraman, S.V., 2009. Prohibitin is a novel regulator of antioxidant response that attenuates colonic inflammation in mice. Gastroenterology 137, 199-208, 208 e191-196. Doi: 10.1053/j.gastro.2009.03.033.

Toledo, F.G., Goodpaster, B.H., 2013. The role of weight loss and exercise in correcting skeletal muscle mitochondrial abnormalities in obesity, diabetes and aging. Mol Cell Endocrinol 379, 30-34. Doi: 10.1016/j.mce.2013.06.018.

Tung, B.T., Rodriguez-Bies, E., Ballesteros-Simarro, M., Motilva, V., Navas, P., Lopez-Lluch, G., 2014. Modulation of endogenous antioxidant activity by resveratrol and exercise in mouse liver is age dependent. J Gerontol A Biol Sci Med Sci 69, 398-409. Doi: 10.1093/gerona/glt102.

Tung, B.T., Rodriguez-Bies, E., Talero, E., Gamero-Estevez, E., Motilva, V., Navas, P., Lopez-Lluch, G., 2015a. Anti-inflammatory effect of resveratrol in old mice liver. Exp Gerontol 64, 1-7. Doi: 10.1016/j.exger.2015.02.004. 
Tung, B.T., Rodriguez-Bies, E., Thanh, H.N., Le-Thi-Thu, H., Navas, P., Sanchez, V.M., Lopez-Lluch, G., 2015b. Organ and tissue-dependent effect of resveratrol and exercise on antioxidant defenses of old mice. Aging Clin Exp Res Doi: 10.1007/s40520-015-0366-8. Doi: 10.1007/s40520-015-0366-8.

Turner, N., Bruce, C.R., Beale, S.M., Hoehn, K.L., So, T., Rolph, M.S., Cooney, G.J., 2007. Excess lipid availability increases mitochondrial fatty acid oxidative capacity in muscle: evidence against a role for reduced fatty acid oxidation in lipid-induced insulin resistance in rodents. Diabetes 56, 2085-2092. Doi: 10.2337/db07-0093.

Twig, G., Elorza, A., Molina, A.J., Mohamed, H., Wikstrom, J.D., Walzer, G., Stiles, L., Haigh, S.E., Katz, S., Las, G., Alroy, J., Wu, M., Py, B.F., Yuan, J., Deeney, J.T., Corkey, B.E., Shirihai, O.S., 2008. Fission and selective fusion govern mitochondrial segregation and elimination by autophagy. EMBO J 27, 433-446. Doi: 10.1038/sj.emboj.7601963.

Vasconsuelo, A., Milanesi, L., Boland, R., 2013. Actions of 17 beta-estradiol and testosterone in the mitochondria and their implications in aging. Ageing Res Rev 12, 907-917. Doi: 10.1016/j.arr.2013.09.001.

Wai, T., Langer, T., 2016. Mitochondrial Dynamics and Metabolic Regulation. Trends Endocrinol Metab 27, 105-117. Doi: 10.1016/j.tem.2015.12.001.

Wallace, D.C., 2012. Mitochondria and cancer. Nat Rev Cancer 12, 685-698. Doi: $10.1038 / n r c 3365$.

Wang, C.H., Wang, C.C., Huang, H.C., Wei, Y.H., 2013. Mitochondrial dysfunction leads to impairment of insulin sensitivity and adiponectin secretion in adipocytes. FEBS J 280, 1039-1050. Doi: 10.1111/febs.12096. 
Weber, T.A., Reichert, A.S., 2010. Impaired quality control of mitochondria: aging from a new perspective. Exp Gerontol 45, 503-511. Doi: 10.1016/j.exger.2010.03.018.

Wullschleger, S., Loewith, R., Hall, M.N., 2006. TOR signaling in growth and metabolism. Cell 124, 471-484. Doi: 10.1016/j.cell.2006.01.016.

Yang, S.B., Tien, A.C., Boddupalli, G., Xu, A.W., Jan, Y.N., Jan, L.Y., 2012. Rapamycin ameliorates age-dependent obesity associated with increased mTOR signaling in hypothalamic POMC neurons. Neuron 75, 425-436. Doi: 10.1016/j.neuron.2012.03.043.

Yang, Y.L., Loh, K.S., Liou, B.Y., Chu, I.H., Kuo, C.J., Chen, H.D., Chen, C.S., 2013. SESN-1 is a positive regulator of lifespan in Caenorhabditis elegans. Exp Gerontol 48, 371-379. Doi: 10.1016/j.exger.2012.12.011.

Yoon, Y., Krueger, E.W., Oswald, B.J., McNiven, M.A., 2003. The mitochondrial protein hFis1 regulates mitochondrial fission in mammalian cells through an interaction with the dynamin-like protein DLP1. Mol Cell Biol 23, 5409-5420. Doi.

Yoon, Y.S., Yoon, D.S., Lim, I.K., Yoon, S.H., Chung, H.Y., Rojo, M., Malka, F., Jou, M.J., Martinou, J.C., Yoon, G., 2006. Formation of elongated giant mitochondria in DFO-induced cellular senescence: involvement of enhanced fusion process through modulation of Fis1. J Cell Physiol 209, 468-480. Doi: 10.1002/jcp.20753.

Yu, J., Nagasu, H., Murakami, T., Hoang, H., Broderick, L., Hoffman, H.M., Horng, T., 2014. Inflammasome activation leads to Caspase-1-dependent mitochondrial damage and block of mitophagy. Proc Natl Acad Sci U S A 111, 15514-15519. Doi: 10.1073/pnas.1414859111. 
Zamboni, M., Mazzali, G., Zoico, E., Harris, T.B., Meigs, J.B., Di Francesco, V., Fantin, F., Bissoli, L., Bosello, O., 2005. Health consequences of obesity in the elderly: a review of four unresolved questions. Int J Obes (Lond) 29, 1011-1029. Doi: $10.1038 /$ sj.ijo.0803005.

Ziegler, D.V., Wiley, C.D., Velarde, M.C., 2015. Mitochondrial effectors of cellular senescence: beyond the free radical theory of aging. Aging Cell 14, 1-7. Doi: 10.1111/acel.12287.

Zorzano, A., Claret, M., 2015. Implications of mitochondrial dynamics on neurodegeneration and on hypothalamic dysfunction. Front Aging Neurosci 7, 101. Doi: $10.3389 /$ fnagi.2015.00101.

Zorzano, A., Liesa, M., Palacin, M., 2009. Role of mitochondrial dynamics proteins in the pathophysiology of obesity and type 2 diabetes. Int $\mathrm{J}$ Biochem Cell Biol 41, 1846-1854. Doi: 10.1016/j.biocel.2009.02.004. 